TRANSACTIONS OF THE

AMERICAN MATHEMATICAL SOCIETY

Volume 364, Number 10, October 2012, Pages 5415-5468

S 0002-9947(2012)05554-1

Article electronically published on May 18, 2012

\title{
HOW TRAVELLING WAVES ATTRACT THE SOLUTIONS OF KPP-TYPE EQUATIONS
}

\author{
MICHAËL BAGES, PATRICK MARTINEZ, AND JEAN-MICHEL ROQUEJOFFRE
}

\begin{abstract}
We consider in this paper a general reaction-diffusion equation of the KPP (Kolmogorov, Petrovskiu, Piskunov) type, posed on an infinite cylinder. Such a model will have a family of pulsating waves of constant speed, larger than a critical speed $c_{*}$. The family of all supercritical waves attracts a large class of initial data, and we try to understand how. We describe in this paper the fate of an initial datum trapped between two supercritical waves of the same velocity: the solution will converge to a whole set of translates of the same wave, and we identify the convergence dynamics as that of an effective drift, around which an effective diffusion process occurs. In several nontrivial particular cases, we are able to describe the dynamics by an effective equation.
\end{abstract}

\section{INTRODUCTION}

The question here is the large-time dynamics of the solutions of reaction-diffusion equations on the whole line or in cylinders, the most general instance being

$$
\left\{\begin{array}{l}
u_{t}-\operatorname{div}(A(x, y) D u)+B(x, y) \cdot D u=f(x, y, u), \quad\left((x, y) \in \mathbb{R} \times \mathbb{T}^{N-1}\right), \\
\lim _{x \rightarrow-\infty} u(t, x, y)=0, \quad \lim _{x \rightarrow+\infty} u(t, x, y)=1 .
\end{array}\right.
$$

Here, $\mathbb{T}^{n}$ denotes the $n$-dimensional torus and $D$ denotes the spatial gradient. The function $f$ will always be supposed to be smooth enough, and positive. Moreover, it will be assumed to be concave in $u$, and moreover:

$$
f(x, y, 0)=f(x, y, 1)=0, \quad \sup _{(x, y)} f_{u}(x, y, 1)<0<\inf _{(x, y)} f_{u}(x, y, 0) .
$$

Additional assumptions on $A, B$ and $f$, such as periodicity in the variable $x$, are required, and we will give a precise statement when needed. More general assumptions (such as less smoothness on $f$, or a condition on the slope of $f$ at 0 instead of the concavity assumption) are possible, but will not be considered. In any case, such a nonlinearity assumption on $f$ will be called a KPP nonlinearity.

It is well known [3] that there exists $c_{*}>0$ such that (1.1) has pulsating wave solutions of speed $c$ if and only if $c \geq c_{*}$. See the general definition in 3]. Here a pulsating wave with speed $c$ will be a solution $\phi_{c}$ of (1.1) such that, for all $(\xi, y) \in \mathbb{R} \times \mathbb{T}^{N-1}$, the function $t \mapsto \phi_{c}(t, \xi-c t, y)$ is $\frac{1}{c}$-periodic. Note that existence results in the spirit of Theorem 0.3 are known, although less complete; see [1] or 9]. The family of all these travelling waves, but not only these, see [13], provides an attractor for a large class of initial data to (1.1). The goal of our work is to understand how this attractor traps the trajectories of (1.1). First we will

Received by the editors May 16, 2010 and, in revised form, November 29, 2010

2010 Mathematics Subject Classification. Primary 35K57; Secondary 35B35, 35K55, 42A38.

(C)2012 American Mathematical Society 
study the most basic model. Next, thanks to the information given by this basic model, we will consider the problem in its full generality. At last, we will consider a periodic one-dimensional case, for which we will be able to give precise answers.

1.1. The basic one-dimensional model. What motivated this study is the following, seemingly innocent question. Consider the most basic 1D model, namely

$$
\left\{\begin{array}{l}
u_{t}-u_{x x}=f(u) \quad(x \in \mathbb{R}), \\
\lim _{x \rightarrow-\infty} u(t, x)=0, \quad \lim _{x \rightarrow+\infty} u(t, x)=1,
\end{array}\right.
$$

with $f$ concave, $f(0)=f(1)=0$. Then, see, for instance, [17, (1.2) has a family of travelling waves; that is, for every $c \geq c_{*}:=2 \sqrt{f^{\prime}(0)}$, there is a unique (up to translation in $x) \phi_{c}$, solving

$$
\begin{cases}c \phi^{\prime}-\phi^{\prime \prime}=f(\phi), & (x \in \mathbb{R}), \\ \lim _{x \rightarrow-\infty} \phi(x)=0, & \lim _{x \rightarrow+\infty} \phi(x)=1 .\end{cases}
$$

In other words $\phi_{c}(x+c t)$ solves (1.2). We ask the question of the stability, under large perturbations, of the supercritical waves, those whose speed is $>c_{*}$. Much is known in this direction; let us extract the following two results.

Theorem 0.1 (Uchiyama [30, Bramson [7]). Let $u_{0}(x)$ be a Cauchy datum for (1.2) such that there is $c>c_{*}$ and $r>0$ for which we have

$$
u_{0}(x)=\phi_{c}(x)\left(1+O\left(e^{r x}\right)\right) \text { as } x \rightarrow-\infty .
$$

Assume moreover that $\limsup _{x \rightarrow+\infty} u_{0}(x)=1$. Then there is some $\omega>0$ such that, we have, as $t \rightarrow+\infty$ :

$$
u(t, x)=\phi_{c}(x+c t)+O\left(e^{-\omega t}\right) .
$$

A much related, and more recent, theorem is

Theorem 0.2 (Berestycki-Hamel [4). Let $u(t, x)$ be a time-global (that is, defined for $t \in \mathbb{R}$ ) solution to (1.2) such that there is $c>c_{*}$ and $M>0$ for which we have

$$
\phi_{c}(x+c t-M) \leq u(t, x) \leq \phi_{c}(x+c t+M) .
$$

Then there is $m \in[-M, M]$ such that $u(t, x)=\phi_{c}(x+c t+m)$.

In fact, the assumption that $u(t, x)$ is trapped between two translates of a wave can be considerably weakened under our assumptions on $f$; see [13.

At first sight, Theorem 0.2 seems to imply the convergence to a wave for any solution starting from a datum trapped between two waves. Not quite, though: Theorem 0.1 only allows perturbations which decay faster than the wave. This seemingly insignificant gap between the two results signals in fact that initial data which are merely sandwiched between two waves, and which do not select an asymptotic wave at $-\infty$, have a wilder behavior. Here is what we can prove.

Theorem 1.1. Let $u_{0}(x)$ be a Cauchy datum for (1.2). Assume the existence of $c>c_{*}$ and $M>0$ such that $\phi_{c}(x-M) \leq u_{0}(x) \leq \phi_{c}(x+M)$. Denote by $r_{-}(c)$ the smallest characteristic exponent at $-\infty$ of (1.3), i.e.,

$$
r_{-}(c)=\frac{c-\sqrt{c^{2}-4 f^{\prime}(0)}}{2} .
$$


Then there is some initial condition $m_{0}$ (given explicitly in the proof), bounded between $-M$ and $M$, and such that, considering the solution $s(t, \xi)$ of

$$
s_{t}-s_{\xi \xi}+\sqrt{c^{2}-c_{*}^{2}} s_{\xi}=0(t>0, \xi \in \mathbb{R}), \quad s(0, \xi)=e^{r_{-}(c) m_{0}(\xi)}
$$

and setting

$m^{a p p}(t, \xi)=\frac{1}{r_{-}(c)} \ln s(t, \xi)=\frac{1}{r_{-}(c)} \ln \left(\frac{1}{\sqrt{4 \pi t}} \int_{\mathbb{R}} e^{\left(\xi-\sqrt{c^{2}-c_{*}^{2}} t-y\right) / 4 t} e^{r_{-}(c) m_{0}(y)} d y\right)$,

we have, as $t \rightarrow+\infty$,

$$
\sup _{x \in \mathbb{R}}\left|u(t, x)-\phi_{c}\left(x+c t+m^{a p p}(t, x+c t)\right)\right|=O\left(\frac{1}{\sqrt{t}}\right) .
$$

Remark 1.2. - The "initial shift" $m_{0}$ is explicit: $m_{0}(x)=\phi_{c}^{-1}\left(u_{0}(x)\right)-x$ if this is a $C^{1}$ bounded and uniformly continuous function, or $m_{0}(x)=$ $\phi_{c}^{-1}(u(1, x))-x$ in any case.

- To our knowledge this result is new, although the arguments used in [7] are very close to proving our theorem.

- In subsection 2.6. we give examples and applications of Theorem 1.1 that allow us to extend in several directions Theorem 0.1:

- the case where the initial shift $m_{0}$ is periodic, hence when the initial condition "oscillates" between two translates of the travelling wave;

- the case where the initial shift $m_{0}$ converges to some constant at $-\infty$ : then we prove the convergence of the solution $u$ to a travelling wave, with a precise convergence rate;

- a case where the solution does not converge to any travelling wave. Indeed, it is known, see [8, 31, that very simple equations such as (1.4) can exhibit complex behaviors; in particular, the $\omega$-limit set (in the sense of uniform convergence on every compact set) can be a whole interval. A related phenomenon for two-dimensional bistable equations was noticed in [26]; the mechanism is somehow different. See [24] for related results in nonlinear supercritical heat equations.

1.2. The general model (1.1). Coming back to the general model (1.1), what we have is of course less precise. We assume that $A, B$ and $f$ satisfy the following additional assumptions:

- $A$ is symmetric, uniformly positive, 1 -periodic with respect to $x$, and $C^{3}\left(\mathbb{R} \times \mathbb{T}^{N-1}\right)$,

- $B$ is 1-periodic with respect to $x, C^{1+\delta}\left(\mathbb{R} \times \mathbb{T}^{N-1}\right)$, and moreover $\operatorname{div} B=0$ and $\int_{(0,1) \times \mathbb{T}^{N-1}} B_{1}=0$ (where $B_{1}$ is the first component of $B$ ),

- $f$ is 1-periodic with respect to $x$.

Let us first state, under the form of a theorem, the basic result that we shall need:

Theorem 0.3 (Berestycki-Hamel [3, Hamel-Roques [14]). There is $c_{*}$ such that (1.1) has no pulsating wave solution if $c<c_{*}$, and a unique, up to translation in $t$, pulsating wave solution if $c \geq c_{*}$. Moreover, for a pulsating wave $\phi_{c}$ we have $\partial_{t} \phi_{c}>0$. 
Existence and monotonicity come from [3], uniqueness comes from [12] and [14]. The theorem corresponding to Theorem 1.1 is:

Theorem 1.3. Let $u_{0}(x, y)$ be a Cauchy datum for (1.1). Assume the existence of $c>c_{*}$ and $M>0$ such that $\phi_{c}(-M, x, y) \leq u_{0}(x, y) \leq \phi_{c}(M, x, y)$. Then there exists a smooth function $m(t, x, y)$, that is solution to a nonlinear parabolic equation with periodic coefficients, such that $\lim _{t \rightarrow+\infty}\left\|\left(m_{t}, D m, D^{2} m\right)(t, . . .)\right\|_{\infty}=0$, and such that

$$
\sup _{(x, y) \in \mathbb{R} \times \mathbb{T}^{N-1}}\left|u(t, x, y)-\phi_{c}(t+m(t, x, y), x, y)\right| \rightarrow 0 \quad \text { as } t \rightarrow+\infty .
$$

As we will see, the shift $m(t, x, y)$ will satisfy, up to a Hopf-Cole transform, a linear diffusion equation (with periodic coefficients). In order to have more insight into its dynamics, we will interpret it in the light of general heat kernel estimates for operators with periodic coefficients, which were proved by Norris 22 at this level of generality. We will see that the underlying processes at work are

- an effective drift $V_{*}(c)$, which can be computed explicitly (and which is, fortunately, consistent with the $1 \mathrm{D}$ expression $\sqrt{c^{2}-c_{*}^{2}}$ !),

- an effective diffusion process around the drift.

Apart from Theorem 1.3 the only multi-dimensional stability results are those of [18] (there we have $A=I$ and $B(x, y)=(\alpha(y), 0)$ ) and [14] for general $A$ and $B$, which prove the asymptotic stability of all the waves under fastly decaying perturbations.

1.3. More precise results concerning the general model (1.1) in one space dimension. We are able to push Theorem 1.3 further for the 1D version of Problem (1.1). It reads, for simplicity, that the matrix $A(x)$ has been set to the identity, but our result would undoubtedly hold without this assumption:

$$
\begin{cases}u_{t}-u_{x x}=f(x, u) & (x \in \mathbb{R}), \\ \lim _{x \rightarrow-\infty} u(t, x)=0, & \lim _{x \rightarrow+\infty} u(t, x)=1 .\end{cases}
$$

Of course the pulsating wave solutions of (1.1) specialize to (1.5); Theorem 0.3 applies and we denote by $c_{*}$ the minimal speed. The additional information of this section is an optimal convergence rate of a solution to (1.5), initially trapped between two waves, to the shifted wave.

Theorem 1.4. Let $u_{0}(x, y)$ be a Cauchy datum for (1.5). Assume the existence of $c>c_{*}$ and $M>0$ such that $\phi_{c}(-M, x, y) \leq u_{0}(x, y) \leq \phi_{c}(M, x, y)$. Then the smooth function $m(t, x, y)$ that is the solution of the nonlinear parabolic equation with periodic coefficients studied in Theorem 1.3 satisfies

$$
\lim _{t \rightarrow+\infty}\left\|\left(m_{t}, m_{x}, m_{x x}\right)(t, .)\right\|_{\infty}=O\left(\frac{1}{\sqrt{t}}\right)
$$

and

$$
\sup _{x \in \mathbb{R}}\left|u(t, x)-\phi_{c}(t+m(t, x), x)\right|=O\left(\frac{1}{\sqrt{t}}\right) \quad \text { as } t \rightarrow+\infty .
$$

The proof of this result, announced in [2], is long and nontrivial; the best part of it consists in retrieving the precise expression of the heat kernel. But it is worth the effort, because it really gives an insight into the heat kernel, and a precise description of the mechanisms at work. It is therefore of independent interest. 
Needless to say, the effective drift is present here, and $V_{*}(c)=\sqrt{c^{2}-c_{*}^{2}}$ when $f(x, u)$ does not depend on $x$.

1.4. Additional comments and open questions. We hope that the ideas developed here will not only provide a better understanding of the dynamics of supercritical KPP waves, but will also help to understand how the critical wave is attained from fastly decaying initial data. In the forthcoming papers [19] and [20, we will see how this works for the 1D homogeneous model (already proved by Bramson [7] but where a deterministic proof is still unknown) and on the multi-D model with special cases of advection. The general case is an important issue that goes far beyond scalar reaction-diffusion equations; see [10].

There are several questions close to this work whose answers would be very interesting:

- Concerning the general model: we could not provide a decay rate estimate about the derivatives of approximate shift, but just the fact that $\lim _{t \rightarrow+\infty}\left\|\left(m_{t}, D m, D^{2} m\right)(t)\right\|_{\infty}=0$, using a contradiction argument. Any decay rate estimate would also immediately provide a decay rate of the uniform convergence as $t \rightarrow+\infty$ of the shifted wave $\phi_{c}(t+m(t, x, y), x, y)$ to the solution $u$ of the Cauchy problem.

- Hence the problem of the convergence rate remains open in the general $\mathrm{N}$ dimensional case. However, in a forthcoming work [19, we will study the case $A=I, B(x, y)=(\alpha(y), 0)$ (thermo-diffusive model for flame propagation) where the techniques are related to the ones used in this paper. We point out that, except in the case of selfadjoint operators, where the heat kernel is known with a lot of precision, see [23, these are the only cases where we can go that far.

- We concentrated our study in the case where the initial condition of the Cauchy problem is trapped between two translates of the same pulsating wave. It would be very interesting to investigate the behavior of the solutions under weakened assumptions on the initial condition. An important first step is taken in Hamel-Nadirashvili [13, where it is proved that (almost) every time-global solution of the $N$-dimensional homogeneous model

$$
u_{t}-\Delta u=u(1-u), \quad t>0, x \in \mathbb{R}^{N}, \quad 0<u<1
$$

is a (possibly uncountably infinite) convex combination of one-dimesional waves. See also the later reference [32].

- The case of initial conditions trapped between two critical waves $\left(c=c_{*}\right)$ is not treated here. This will be studied in the forthcoming paper [20].

1.5. Plan of the paper. The plan of the paper is the following. In Section 2 we prove Theorem 1.1, the argument, although quite simple, will be explained at length because its main lines will always be the same in the more complicated subsequent cases. We will also apply our method and result on several natural examples. In Section 3 we prove the general Theorem 1.3 for model (1.1). This will allow us to prove a Liouville theorem very close in spirit to an elliptic result proved by Rossi in his thesis 27. We will also provide the interpretation of the nonlinear diffusion equation for $m$ in terms of the general heat kernel estimates. Finally, in Section 4 . we deal with the general $1 \mathrm{D}$ model (1.5). At this stage the reader will be supposed to know the argument, and only the nonlinear diffusion equation for $m$ will be 
treated. As said above, the argument is rather long; it will be broken into two parts with the hope that it will be more amenable to the interested reader. Finally, an appendix describes some elementary but useful, and not so easy, estimates on Gaussian-type integrals.

\section{The Basic 1D model: Proof of Theorem 1.1}

This part is devoted to the proof of Theorem 1.1 and, although most of the tools displayed here are quite standard, the chain of arguments is not.

2.1. The travelling wave of speed $c$. We consider the classical change of variables $(t, x) \mapsto(t, \xi=x+c t)$. Now if $u(t, x)$ is a solution of (1.2), the function $\widetilde{u}$ defined by

$$
\widetilde{u}(t, \xi):=u(t, \xi-c t)
$$

satisfies $u(t, x)=\widetilde{u}(t, x+c t)$ and thus is a solution of

$$
\left\{\begin{array}{l}
\widetilde{u}_{t}+c \widetilde{u}_{\xi}-\widetilde{u}_{\xi \xi}=f(\widetilde{u}), \quad t>0, \xi \in \mathbb{R}, \\
\widetilde{u}(0, \xi)=u(0, \xi)=u_{0}(\xi) .
\end{array}\right.
$$

Then consider the differential operator

$$
N L_{c}[\widetilde{U}]=\widetilde{U}_{t}+c \widetilde{U}_{\xi}-\widetilde{U}_{\xi \xi}-f(\widetilde{U}) .
$$

(Hence $\widetilde{u}$ is a solution of the differential equation of (2.1) if and only if $N L_{c}[\widetilde{u}]=0$.) Throughout the section we assume that $c>c_{*}=2 \sqrt{f^{\prime}(0)}$. Let $r_{ \pm}(c)$ be the characteristic exponents at $-\infty$ of (1.3), i.e.

$$
r_{ \pm}(c)=\frac{c \pm \sqrt{c^{2}-4 f^{\prime}(0)}}{2}
$$

We recall that, for a given wave $\phi_{c}$, there is $q>0$ and $\delta>0$ such that, as $\xi \rightarrow-\infty$,

$$
\begin{gathered}
\phi_{c}(\xi)=q e^{r_{-}(c) \xi}+O\left(e^{\left(r_{-}(c)+\delta\right) \xi}\right), \phi_{c}^{\prime}(\xi)=q r_{-}(c) e^{r_{-}(c) \xi}+O\left(e^{\left(r_{-}(c)+\delta\right) \xi}\right), \\
\phi_{c}^{\prime \prime}(\xi)=q r_{-}^{2}(c) e^{r_{-}(c) \xi}+O\left(e^{\left(r_{-}(c)+\delta\right) \xi}\right) .
\end{gathered}
$$

2.2. Exact local shift. Given a sufficiently smooth function $m:(0,+\infty) \times \mathbb{R} \rightarrow \mathbb{R}$, consider

$$
T^{(m)} \phi_{c}(t, \xi):=\phi_{c}(\xi+m(t, \xi)) .
$$

Of course when $m$ is identically zero, we have $T^{(0)} \phi_{c}(t, \xi):=\phi_{c}(\xi)$, and $T^{(0)} \phi_{c}$ satisfies $N L_{c}\left[T^{(0)} \phi_{c}\right]=0$. More generally, we would like to know what conditions on $m$ imply that $T^{(m)} \phi_{c}$ is a solution of (2.1). We have

$N L_{c}\left[T^{(m)} \phi_{c}\right]=\left(m_{t}-m_{\xi \xi}-c\left(m_{\xi}+m_{\xi}^{2}\right)\right) \phi_{c}^{\prime}(\xi+m(t, \xi))+\left(2 m_{\xi}+m_{\xi}^{2}\right) f\left(\phi_{c}(\xi+m(t, \xi))\right)$.

Since $\phi_{c}$ is strictly increasing, we can consider the exact shift

$$
m^{*}(t, \xi):=\phi_{c}^{-1}(\widetilde{u}(t, \xi))-\xi
$$

some computations show that $m^{*}$ satisfies the following nonlinear parabolic equation:

$$
\left\{\begin{array}{l}
\left(m_{t}^{*}-m_{\xi \xi}^{*}-c\left(m_{\xi}^{*}+m_{\xi}^{* 2}\right)\right) \phi_{c}^{\prime}\left(\xi+m^{*}(t, \xi)\right) \\
+\left(2 m_{\xi}^{*}+m_{\xi}^{* 2}\right) f\left(\phi_{c}\left(\xi+m^{*}(t, \xi)\right)\right)=0 \\
m^{*}(0, \xi)=\phi_{c}^{-1}\left(u_{0}(\xi)\right)-\xi=: m_{0}^{*}(\xi)
\end{array}\right.
$$


To study the solution of this problem seems difficult. Hence our strategy will be

- to find a parabolic problem that will be as close as possible to the previous one, but simpler; this will allow us to study the properties of its solution $m$, which we will call the "approximate shift", "approximate" meaning that it is a solution of a problem "close" to the one satisfied by the exact shift $m^{*}$; of course in this case the functions $\widetilde{u}$ and $T^{(m)} \phi_{c}$ will have no reason to coincide;

- then to consider the difference $\widetilde{u}-T^{(m)} \phi_{c}$, and to estimate its asymptotic behavior as $t \rightarrow+\infty$.

What came as a surprise to us is that this very simple and natural strategy actually enables us to say something about very general models.

Even if we cannot say many things on the exact shift $m^{*}(t, \xi)=\phi_{c}^{-1}(\widetilde{u}(t, \xi))-\xi$, we can see that it has the following property: for all $t>0, m^{*}(t, \cdot)$ is of class $C^{1}(\mathbb{R})$ and is bounded in the natural $C^{1}$-norm: indeed,

- it is clear that $m^{*}$ is bounded, and more precisely, $m^{*}(t, \xi) \in[-M, M]$; this follows from the assumption that $u_{0}(\xi) \in\left[\phi_{c}(\xi-M), \phi_{c}(\xi+M)\right]$, using the weak maximum principle;

- next, from parabolic regularity, $m^{*}(t, \cdot)$ is of class $C^{1}(\mathbb{R})$;

- at last,

$$
m_{\xi}^{*}(t, \xi)=\frac{\widetilde{u}_{\xi}(t, \xi)}{\phi_{c}^{\prime}\left(\xi+m^{*}(t, \xi)\right)}-1=\frac{\widetilde{u}_{\xi}(t, \xi)}{\phi_{c}^{\prime}(\xi)} \frac{\phi_{c}^{\prime}(\xi)}{\phi_{c}^{\prime}\left(\xi+m^{*}(t, \xi)\right)}-1
$$

hence $m_{\xi}^{*}(t, \cdot)$ is bounded if and only if $\frac{\widetilde{u}_{\xi}}{\phi_{c}^{\prime}}$ is bounded, and it follows from local parabolic estimates that this is true, once again using the fact that the weak maximum principle implies that $\phi_{c}(\xi-M) \leq \widetilde{u}(t, \xi) \leq \phi_{c}(\xi+M)$ for all $t>0$ and all $\xi \in \mathbb{R}$.

Hence, in particular, $m^{*}(1, \cdot)$ is of class $C^{1}(\mathbb{R})$ and is bounded in the natural $C^{1}$-norm. We will use this remark in the following.

2.3. Approximate shift. If $m$ is bounded, remark that the coefficients $\phi_{c}^{\prime}(\xi+m(t, \xi))$ and $f\left(\phi_{c}(\xi+m(t, \xi))\right)$ appearing in $N L_{c}\left[T^{(m)} \phi_{c}\right]$ decay as $e^{r_{-}(c) \xi}$ as $\xi \rightarrow-\infty$. Now let us ask if we can choose $m$ so that we get a better decay for $N L_{c}\left[T^{(m)} \phi_{c}\right]$, a property that would help us a lot in estimating the difference between $\widetilde{u}$ and the associated shifted wave, as will be clearer later (see subsection 2.5). For this purpose, we linearize the equation at $-\infty$, studying the behavior of the coefficients $\phi_{c}^{\prime}(\xi+m(t, \xi))$ and $f\left(\phi_{c}(\xi+m(t, \xi))\right)$ as $\xi \rightarrow-\infty$. Obviously we have

$$
\begin{aligned}
f\left(\phi_{c}(\xi)\right) & =f^{\prime}(0) \phi_{c}(\xi)+\left(f\left(\phi_{c}(\xi)\right)-f^{\prime}(0) \phi_{c}(\xi)\right) \\
& =\frac{f^{\prime}(0)}{r_{-}(c)} \phi_{c}^{\prime}(\xi)+\left(f\left(\phi_{c}(\xi)\right)-\frac{f^{\prime}(0)}{r_{-}(c)} \phi_{c}^{\prime}(\xi)\right)
\end{aligned}
$$

denote

$$
g(t, \xi):=f\left(\phi_{c}(\xi+m(t, \xi))\right)-\frac{f^{\prime}(0)}{r_{-}(c)} \phi_{c}^{\prime}(\xi+m(t, \xi))
$$


then we have

$$
\begin{aligned}
N L_{c}\left[T^{(m)} \phi_{c}\right]=\left(m_{t}-\right. & \left.m_{\xi \xi}-c\left(m_{\xi}+m_{\xi}^{2}\right)\right) \phi_{c}^{\prime}(\xi+m(t, \xi)) \\
& +\left(2 m_{\xi}+m_{\xi}^{2}\right)\left(\frac{f^{\prime}(0)}{r_{-}(c)} \phi_{c}^{\prime}(\xi+m(t, \xi))+\left(2 m_{\xi}+m_{\xi}^{2}\right) g(t, \xi) .\right.
\end{aligned}
$$

Since

$$
-c+2 \frac{f^{\prime}(0)}{r_{-}(c)}=\sqrt{c^{2}-c_{*}^{2}} \quad \text { and } \quad-c+\frac{f^{\prime}(0)}{r_{-}(c)}=-r_{-}(c),
$$

we obtain

$N L_{c}\left[T^{(m)} \phi_{c}\right]=\left(m_{t}-m_{\xi \xi}+\sqrt{c^{2}-c_{*}^{2}} m_{\xi}-r_{-}(c) m_{\xi}^{2}\right) \phi_{c}^{\prime}(\xi+m(t, \xi))+\left(2 m_{\xi}+m_{\xi}^{2}\right) g(t, \xi)$.

When $\xi \rightarrow-\infty$, we have $f\left(\phi_{c}(\xi)\right)-\frac{f^{\prime}(0)}{r_{-}(c)} \phi_{c}^{\prime}(\xi)=O\left(e^{\left(r_{-}(c)+\delta\right) \xi}\right)$ with some $\delta>0$; hence $g$ has the same property if $m$ is bounded. This makes natural the choice of $m$ : take it to solve

$$
m_{t}-m_{\xi \xi}+\sqrt{c^{2}-c_{*}^{2}} m_{\xi}-r_{-}(c) m_{\xi}^{2}=0,
$$

with a suitable initial condition:

- if $m_{0}^{*}(\xi)=\phi_{c}^{-1}\left(u_{0}(\xi)\right)-\xi$ is of class $C^{1}(\mathbb{R})$ and bounded in norm $C^{1}$, then we choose $m$ as the solution of

$$
\left\{\begin{array}{l}
m_{t}-m_{\xi \xi}+\sqrt{c^{2}-c_{*}^{2}} m_{\xi}-r_{-}(c) m_{\xi}^{2}=0, \quad t>0, \xi \in \mathbb{R}, \\
m(0, \xi)=m_{0}^{*}(\xi), \quad \xi \in \mathbb{R}
\end{array}\right.
$$

- in the general case, as we noted that $m^{*}(1, \cdot)$ is of class $C^{1}(\mathbb{R})$ and bounded in norm $C^{1}$, then we can choose $m$ as the solution of

$$
\left\{\begin{array}{l}
m_{t}-m_{\xi \xi}+\sqrt{c^{2}-c_{*}^{2}} m_{\xi}-r_{-}(c) m_{\xi}^{2}=0, \quad t>1, \quad \xi \in \mathbb{R}, \\
m(1, \xi)=m^{*}(1, \xi), \quad \xi \in \mathbb{R} .
\end{array}\right.
$$

In the following we assume that we are in the first case, that is, that $m$ is a solution of (2.4). The study of the second case is the same, the only difference being that $t \in(1,+\infty)$ instead of $(0,+\infty)$.

2.4. The properties of the approximate shift. There is an easy expression for $m$ : its Hopf-Cole transform $s(t, \xi)=e^{r_{-}(c) m(t, \xi)}$ solves

$$
\left\{\begin{array}{l}
s_{t}-s_{\xi \xi}+\sqrt{c^{2}-c_{*}^{2}} s_{\xi}=0, \quad t>0, \xi \in \mathbb{R}, \\
s(0, \xi)=e^{r_{-}(c) m_{0}^{*}(\xi)}=: s_{0}(\xi), \quad \xi \in \mathbb{R},
\end{array}\right.
$$

and thus the function $S$, defined by

$$
S(t, \xi):=s\left(t, \xi+\sqrt{c^{2}-c_{*}^{2}} t\right),
$$

is a solution of the heat equation

$$
\left\{\begin{array}{l}
S_{t}-S_{\xi \xi}=0, \quad t>0, \xi \in \mathbb{R} \\
S(0, \xi)=s_{0}(\xi), \quad \xi \in \mathbb{R} .
\end{array}\right.
$$


Hence, if $G(t, \xi)$ is the heat kernel $\frac{1}{\sqrt{4 \pi t}} e^{-\frac{\xi^{2}}{4 t}}$, then

$$
\begin{gathered}
S(t, \xi)=\int_{\mathbb{R}} G(t, \xi-y) s_{0}(y) d y \\
s(t, \xi)=S\left(t, \xi-\sqrt{c^{2}-c_{*}^{2}} t\right)=\int_{\mathbb{R}} G\left(t, \xi-\sqrt{c^{2}-c_{*}^{2}} t-y\right) s_{0}(y) d y,
\end{gathered}
$$

and finally

$$
m(t, \xi)=\frac{1}{r_{-}(c)} \ln s(t, \xi)=\frac{1}{r_{-}(c)} \ln \left(\int_{\mathbb{R}} G\left(t, \xi-\sqrt{c^{2}-c_{*}^{2}} t-y\right) e^{r_{-}(c) m_{0}^{*}(y)} d y\right) .
$$

This is exactly the expression in Theorem 1.1, choosing $m^{a p p}=m$. We deduce the following properties, useful in the sequel:

- first, $m$ is bounded, and more precisely, $m(t, \xi) \in[-M, M]$ for all $t \geq 0$ and all $\xi \in \mathbb{R}$ : indeed, this is true at $t=0$ and remains true thanks to the weak maximum principle or directly from the formula (2.8);

- its spatial derivative satisfies: for all $t>0,\left\|m_{\xi}(t, .)\right\|_{\infty}=O\left(\frac{1}{1+\sqrt{t}}\right)$; indeed,

$$
m_{\xi}(t, \xi)=\frac{1}{r_{-}(c)} \frac{S_{\xi}\left(t, \xi-\sqrt{c^{2}-c_{*}^{2}} t\right)}{S\left(t, \xi-\sqrt{c^{2}-c_{*}^{2}} t\right)},
$$

and, for all $t>0$ and $\xi \in \mathbb{R}$, we have

$$
\left|S_{\xi}(t, \xi)\right| \leq C\left\|s_{0}^{\prime}\right\|_{\infty} \quad \text { and } \quad\left|S_{\xi}(t, \xi)\right| \leq C \frac{1}{\sqrt{t}}\left\|s_{0}\right\|_{\infty} ;
$$

hence we obtain what we claimed;

- note that, in the same way, $\left\|m_{t}(t, .)\right\|_{\infty}=O\left(\frac{1}{\sqrt{t}}\right)$ and $\left\|m_{\xi \xi}(t, .)\right\|_{\infty}=O\left(\frac{1}{t}\right)$ for all $t>0$.

Now we denote by $\widetilde{u}^{a p p}$ the associated shifted wave:

$$
\widetilde{u}^{a p p}(t, \xi):=T^{(m)} \phi_{c}=\phi_{c}(\xi+m(t, \xi)) .
$$

It remains to prove that we have grabbed the correct shift, that is, that $\| \widetilde{u}(t,)-$. $\widetilde{u}^{a p p}(t,.) \|_{\infty}=O\left(\frac{1}{\sqrt{t}}\right)$ as $t \rightarrow+\infty$, and Theorem 1.1 will be proved.

\subsection{The difference between the solution and the shifted wave.}

2.5.1. Solutions decaying sufficiently fast in space will decay exponentially in time. The following (quite standard) lemma will be useful to estimate the difference between the solution and the shifted wave.

Lemma 2.1. Let $v(t, \xi)$ solve

$$
v_{t}-v_{\xi \xi}+c v_{\xi}-f^{\prime}(0) v=0(t>0, \xi \in \mathbb{R}), \quad v(0, \xi)=v_{0}(\xi)
$$

with $v_{0}$ bounded and uniformly continuous on $\mathbb{R}$. Assume additionally the existence of $\delta \in\left(0, r_{+}(c)-r_{-}(c)\right)$ such that

$$
v_{0}(\xi)=O\left(e^{\left(r_{-}(c)+\delta\right) \xi}\right) \quad \text { as } \xi \rightarrow-\infty .
$$

Then there is $\omega(\delta)>0$ such that

$$
|v(t, \xi)| \leq e^{\left(r_{-}(c)+\delta\right) \xi} e^{-\omega(\delta) t} \sup _{z \in \mathbb{R}}\left|e^{-\left(r_{-}(c)+\delta\right) z} v_{0}(z)\right| .
$$


Proof of Lemma 2.1, Fix $d>0$ and consider $V(t, \xi)=v(t, \xi) e^{-d \xi}$. Then $V$ solves

$$
\left\{\begin{array}{l}
V_{t}+(c-2 d) V_{\xi}-V_{\xi \xi}+\left(c d-d^{2}-f^{\prime}(0)\right) V=0, \quad t>0, \xi \in \mathbb{R}, \\
V(0, \xi)=v_{0}(\xi) e^{-d \xi}
\end{array}\right.
$$

Now we note that $c d-d^{2}-f^{\prime}(0)>0$ if $d \in\left(r_{-}(c), r_{+}(c)\right)$, and $V(0,$.$) is bounded if$ $r_{-}(c)+\delta-d \geq 0$. Hence we choose $d \in\left(r_{-}(c), r_{-}(c)+\delta\right]$; then $c d-d^{2}-f^{\prime}(0)>0$, and the weak maximum principle implies that

$$
|V(t, \xi)| \leq\|V(0, .)\|_{\infty} e^{-\left(c d-d^{2}-f^{\prime}(0)\right) t},
$$

which implies that

$$
|v(t, \xi)| \leq\left(\sup _{z \in \mathbb{R}}\left|v_{0}(z) e^{-d z}\right|\right) e^{d \xi} e^{-\left(c d-d^{2}-f^{\prime}(0)\right) t},
$$

which is Lemma 2.1 choosing $d=r_{-}(c)+\delta$ and $\omega(\delta)=c d-d^{2}-f^{\prime}(0)$.

2.5.2. Application: Proof of Theorem 1.1. With this in hand, we may complete the proof of Theorem 1.1], proving that $\left\|\widetilde{u}(t, .)-\widetilde{u}^{a p p}(t, .)\right\|_{\infty}=O\left(\frac{1}{\sqrt{t}}\right)$ as $t \rightarrow+\infty$.

First, note that (2.3) and the choice of the shift $m$ imply that

$$
N L_{c}\left[\widetilde{u}^{a p p}\right]=\left(2 m_{\xi}+m_{\xi}^{2}\right) g(t, \xi) .
$$

Denote

$$
a(t, \xi):=-\frac{f(\widetilde{u}(t, \xi))-f\left(\widetilde{u}^{a p p}(t, \xi)\right)}{\widetilde{u}(t, \xi)-\widetilde{u}^{a p p}(t, \xi)} .
$$

Since $f$ is concave, we have

$$
-f^{\prime}(0) \leq a(t, \xi) \leq-f^{\prime}(1) .
$$

Of course, the interest in introducing this function $a$ comes from the following fact:

$$
\begin{aligned}
N L_{c}[\widetilde{u}]-N L_{c}\left[\widetilde{u}^{a p p}\right]= & -N L_{c}\left[\widetilde{u}^{a p p}\right] \\
= & \left(\widetilde{u}_{t}+c \widetilde{u}_{\xi}-\widetilde{u}_{\xi \xi}-f(\widetilde{u})\right)-\left(\widetilde{u}_{t}^{a p p}+c \widetilde{u}_{\xi}^{a p p}-\widetilde{u}_{\xi \xi}^{a p p}-f\left(\widetilde{u}^{a p p}\right)\right) \\
= & \left(\widetilde{u}-\widetilde{u}^{a p p}\right)_{t}+c\left(\widetilde{u}-\widetilde{u}^{a p p}\right)_{\xi} \\
& -\left(\widetilde{u}-\widetilde{u}^{a p p}\right)_{\xi \xi}-\left(f(\widetilde{u})-f\left(\widetilde{u}^{a p p}\right)\right) \\
= & \left(\widetilde{u}-\widetilde{u}^{a p p}\right)_{t}+c\left(\widetilde{u}-\widetilde{u}^{a p p}\right)_{\xi} \\
& -\left(\widetilde{u}-\widetilde{u}^{a p p}\right)_{\xi \xi}+a(t, \xi)\left(\widetilde{u}-\widetilde{u}^{a p p}\right):=g_{1}(t, \xi) .
\end{aligned}
$$

Let $\widetilde{w}(t, \xi)$ solve

$$
\left\{\begin{array}{l}
\widetilde{w}_{t}+c \widetilde{w}_{\xi}-\widetilde{w}_{\xi \xi}-f^{\prime}(0) \widetilde{w}=\left|g_{1}(t, \xi)\right|, \\
\widetilde{w}(0, \xi)=\left|\widetilde{u}-\widetilde{u}^{a p p}\right|(0, \xi)=\left|u_{0}(\xi)-\phi_{c}\left(\xi+m_{0}^{*}(\xi)\right)\right|=0 .
\end{array}\right.
$$

We are going to prove the following facts:

Claim 2.2. For all $t>0$ and all $\xi \in \mathbb{R}$, we have $\left|\widetilde{u}-\widetilde{u}^{a p p}\right|(t, \xi) \leq \widetilde{w}(t, \xi)$.

Claim 2.3. For all $\xi_{0} \in \mathbb{R}, \sup _{\xi \leq \xi_{0}} \widetilde{w}(t, \xi)=O\left(\frac{1}{1+\sqrt{t}}\right)$.

Claim 2.4. There exists $\xi_{0} \in \mathbb{R}$ such that $\sup _{\xi \geq \xi_{0}}\left|\widetilde{u}-\widetilde{u}^{\text {app }}\right|(t, \xi)=O\left(\frac{1}{1+\sqrt{t}}\right)$. 
It is clear that Claims 2.2 2.4 imply Theorem 1.1 Choosing $\xi_{0}$ given in Claim 2.4 we obtain using Claims 2.2 and 2.3

$$
\sup _{\xi \leq \xi_{0}}\left|\widetilde{u}-\widetilde{u}^{a p p}\right|(t, \xi) \leq \sup _{\xi \leq \xi_{0}} \widetilde{w}(t, \xi)=O\left(\frac{1}{1+\sqrt{t}}\right),
$$

and adding Claim 2.4, we obtain that

$$
\sup _{\xi \in \mathbb{R}}\left|\widetilde{u}-\widetilde{u}^{a p p}\right|(t, \xi)=O\left(\frac{1}{1+\sqrt{t}}\right) .
$$

Hence it remains to prove these claims.

Claim 2.2 is a consequence of the weak maximum principle. Indeed, first $\widetilde{w}$ is nonnegative; next, $\widetilde{u}-\widetilde{u}^{a p p}-\widetilde{w}$ satisfies

$$
\begin{aligned}
\left(\widetilde{u}-\widetilde{u}^{a p p}-\widetilde{w}\right)_{t}+c\left(\widetilde{u}-\widetilde{u}^{a p p}-\widetilde{w}\right)_{\xi}-\left(\widetilde{u}-\widetilde{u}^{a p p}-\widetilde{w}\right)_{\xi \xi} \\
+a\left(\widetilde{u}-\widetilde{u}^{a p p}-\widetilde{w}\right)=G-|G|-\left(a+f^{\prime}(0)\right) \widetilde{w} \leq 0
\end{aligned}
$$

and

$$
\left(\widetilde{u}-\widetilde{u}^{a p p}-\widetilde{w}\right)(0, \xi)=0
$$

hence $\widetilde{u}-\widetilde{u}^{a p p}-\widetilde{w} \leq 0$. In the same way, $\widetilde{u}-\widetilde{u}^{a p p}+\widetilde{w} \geq 0$; hence Claim 2.2 is proved.

Claim 2.3 is a consequence of Lemma 2.1. by Duhamel's formula,

$$
\widetilde{w}(t, \xi)=\int_{0}^{t} e^{-(t-s)\left(-\partial_{\xi \xi}+c \partial_{\xi}-f^{\prime}(0)\right)}\left|g_{1}(s, .)\right| d s ;
$$

since

$$
g_{1}(t, \xi)=-\left(2 m_{\xi}+m_{\xi}^{2}\right)\left(f\left(\phi_{c}(\xi+m(t, \xi))\right)-\frac{f^{\prime}(0)}{r_{-}(c)} \phi_{c}^{\prime}(\xi+m(t, \xi))\right),
$$

there is some $\delta \in\left(0, r_{+}(c)-r_{-}(c)\right)$ and $C_{0}>0$ such that, for $t>0$ and $\xi \in \mathbb{R}$,

$$
\left|g_{1}(t, \xi)\right| \leq \frac{C_{0}}{1+\sqrt{t}} \frac{1}{1+e^{-\left(r_{-}(c)+\delta\right) \xi}} .
$$

Then Lemma 2.1 implies that

$$
e^{-(t-s)\left(-\partial_{\xi \xi}+c \partial_{\xi}-f^{\prime}(0)\right)}\left|g_{1}(s, .)\right|(\xi) \leq e^{\left(r_{-}(c)+\delta\right) \xi} e^{-\omega(\delta)(t-s)} \frac{C_{0}}{1+\sqrt{s}} .
$$

Then

$$
|\widetilde{w}(t, \xi)| \leq C_{0} e^{\left(r_{-}(c)+\delta\right) \xi} \int_{0}^{t} e^{-\omega(\delta)(t-s)} \frac{d s}{1+\sqrt{s}} .
$$

Since

$$
\int_{0}^{t} \frac{e^{\omega(\delta) s}}{1+\sqrt{s}} d s \sim_{t \rightarrow+\infty} \frac{1}{\omega(\delta)} \frac{e^{\omega(\delta) t}}{1+\sqrt{t}},
$$

we obtain the following expression, valid for all $(t, \xi) \in \mathbb{R}_{+} \times \mathbb{R}$ :

$$
|\widetilde{w}(t, \xi)| \leq \frac{C_{1}}{1+\sqrt{t}} e^{\left(r_{-}(c)+\delta\right) \xi} ;
$$

hence Claim 2.3 is proved.

Claim 2.4 is a consequence of Claim 2.3, using the following classical argument: first, there is some $q_{0}>0$ and $\eta>0$ such that $-f^{\prime}(y) \geq q_{0}$ for all $y \in(1-\eta, 1)$; choose $\xi_{0}$ such that $\phi_{c}\left(\xi_{0}-M\right)>1-\eta$; next, because $u_{0}$ is trapped between two waves of the same speed, this order is preserved for all time; hence $\widetilde{u}(t, \xi) \geq$ $\phi_{c}(\xi-M)$ for all $t>0$ and all $\xi \in \mathbb{R}$; at last, since we already know that the shift $m$ 
is bounded between $-M$ and $M$, the mean value theorem implies that $a(t, \xi) \geq q_{0}$ for all $t \geq 0$ and $\xi \geq \xi_{0}$. Thus the difference $\widetilde{u}-\widetilde{u}^{a p p}$ satisfies

$$
\begin{cases}\left(\widetilde{u}-\widetilde{u}^{a p p}\right)_{t}+c\left(\widetilde{u}-\widetilde{u}^{a p p}\right)_{\xi}-\left(\widetilde{u}-\widetilde{u}^{a p p}\right)_{\xi \xi}+a(t, \xi)\left(\widetilde{u}-\widetilde{u}^{a p p}\right)=O\left(\frac{1}{1+\sqrt{t}}\right), & t>0, \xi \geq \xi_{0}, \\ \left(\widetilde{u}-\widetilde{u}^{a p p}\right)\left(t, \xi_{0}\right)=O\left(\frac{1}{1+\sqrt{t}}\right), \quad t>0, & \\ \left(\widetilde{u}-\widetilde{u}^{a p p}\right)(0, \xi)=0, \quad \xi \geq \xi_{0} . & \end{cases}
$$

The function $\frac{C}{\sqrt{1+\varepsilon t}}$ is a supersolution if $C$ is sufficiently large and $\varepsilon$ is sufficiently small; hence the weak maximum principle implies Claim 2.4

2.6. Examples and comparison with the literature. The goal of this subsection is to study some typical examples. We are going to examine

- the case where $m_{0}$ is periodic, which is not covered by the existing literature: we will prove that the associated solution $u$ of (1.2) converges to a translate of the travelling wave, but not the one that could be expected; see subsection 2.6.1 and Proposition 2.5.

- a case where $m_{0}$ oscillates between two values: we will see that $m_{0}$ can be chosen so that the associated solution $u$ of (1.2) does not converge to any translate of the travelling wave; see subsection 2.6.2 and Proposition 2.7

- the typical case where $m_{0}$ converges to some constant $m_{0}(-\infty)$ as $x \rightarrow$ $-\infty$ : in this case, Theorem 0.1 applies when additionally the convergence is exponentially fast. We will prove that the associated solution $u$ of (1.2) converges to a translate of the travelling wave, and more precisely the one that is expected: $\phi_{c}\left(x+c t+m_{0}(-\infty)\right)$, with a precise rate of convergence, roughly speaking the rate of convergence of $m_{0}$ to its limit $m_{0}(-\infty)$; see subsections 2.6.3 and 2.6.4.

Everything is based on the fact that we have an explicit formula for the approximate shift $m(t, \xi)$, obtained in (2.8).

2.6.1. The case where the initial shift $m_{0}$ is periodic. Assume that the initial shift is $T$-periodic, and denote by $\left\langle m_{0}\right\rangle$ its mean value:

$$
\left\langle m_{0}\right\rangle=\frac{1}{T} \int_{0}^{T} m_{0}
$$

Proposition 2.5. The solution $u$ of (1.2) satisfies

(2.12) $\sup _{x \in \mathbb{R}}\left|u(t, x)-\phi_{c}\left(x+c t+\mu_{0}\right)\right|=O\left(\frac{1}{\sqrt{t}}\right), \quad$ where $\quad \mu_{0}=\frac{1}{r_{-}(c)} \ln \left\langle e^{r_{-}(c) m_{0}}\right\rangle$.

Remark 2.6. One could have expected the convergence of the solution $u$ of (1.2) to the travelling wave $\phi_{c}\left(x+c t+\left\langle m_{0}\right\rangle\right)$. However, this is not the case, since in general $\mu_{0} \neq\left\langle m_{0}\right\rangle$.

Proof of Proposition 2.5. Since the function $S_{0}-\left\langle S_{0}\right\rangle$ is periodic and has mean value zero, the function

$$
S_{1}(\xi):=\int_{0}^{\xi}\left(S_{0}(y)-\left\langle S_{0}\right\rangle\right) d y
$$


is bounded. Then, thanks to an integration by parts,

$$
S(t, \xi)=\frac{1}{\sqrt{4 \pi t}} \int_{\mathbb{R}} e^{-(\xi-y)^{2} /(4 t)} S_{0}(y) d y=\left\langle S_{0}\right\rangle+\frac{1}{\sqrt{\pi t}} \int_{\mathbb{R}} \sigma e^{-\sigma^{2}} S_{1}(\xi+2 \sigma \sqrt{t}) d \sigma .
$$

We obtain that

$$
\left|S(t, \xi)-\left\langle S_{0}\right\rangle\right| \leq \frac{1}{\sqrt{\pi t}}\left\|S_{1}\right\|_{\infty}
$$

From

$$
m(t, \xi)=\frac{1}{r_{-}(c)} \ln S\left(t, \xi-\sqrt{c^{2}-c_{*}^{2}} t\right)
$$

we have

$$
\sup _{\xi \in \mathbb{R}}\left|m(t, \xi)-\frac{1}{r_{-}(c)} \ln \left\langle S_{0}\right\rangle\right|=O\left(\frac{1}{\sqrt{t}}\right) .
$$

Then

$$
\tilde{u}^{a p p}(t, \xi)-\phi_{c}\left(\xi+\mu_{0}\right)=\phi_{c}(\xi+m(t, \xi))-\phi_{c}\left(\xi+\mu_{0}\right)=O\left(m(t, \xi)-\mu_{0}\right),
$$

which implies that

$$
\sup _{\xi \in \mathbb{R}}\left|\tilde{u}^{a p p}(t, \xi)-\phi_{c}\left(\xi+\mu_{0}\right)\right|=O\left(\frac{1}{\sqrt{t}}\right),
$$

and (2.12) follows from Theorem 1.1 .

2.6.2. An example where there is no convergence to some translate of the travelling wave. We may deduce the following proposition from [31, but its proof is elementary enough to be displayed here. Moreover, see the conclusion of Section 3 below, it is valid in the multi-D inhomogeneous case, not treated in [31.

Proposition 2.7. Assume that the initial shift $m_{0}$ is such that $S_{0}=e^{r_{-}(c) m_{0}}$ oscillates slowly at $-\infty$ :

$\forall \varepsilon>0, \exists A>0, \exists \eta>0, \forall z, z^{\prime} \leq-A$ and $1-\eta \leq \frac{z}{z^{\prime}} \leq 1+\eta \Longrightarrow\left|S_{0}(z)-S_{0}\left(z^{\prime}\right)\right| \leq \varepsilon$.

Then the Hopf-Cole transform of the approximate shift $m$ satisfies the following:

$$
s(t, 0)=S_{0}\left(-\sqrt{c^{2}-c_{*}^{2}} t\right)+o(1)
$$

As a consequence, if $S_{0}$ slowly oscillates but does not have a limit at $-\infty$, then neither $s(t, 0)$ nor the approximate shift $m(t, 0)$ converge to a constant, and the solution $u$ does not converge to a translate of the travelling wave.

Remark 2.8. For example, the function

$$
S_{0}(x)=2+\cos \ln \left(1+x^{2}\right)
$$

oscillates slowly and does not have a limit at $-\infty$.

Proof of Proposition 2.7. We recall that

$$
m(t, \xi)=\frac{1}{r_{-}(c)} \ln s(t, \xi)=\frac{1}{r_{-}(c)} \ln S\left(t, \xi-\sqrt{c^{2}-c_{*}^{2}} t\right)
$$


and we fix $\xi=0$. We have

$$
\begin{gathered}
S\left(t,-\sqrt{c^{2}-c_{*}^{2}} t\right)=\frac{1}{\sqrt{4 \pi t}} \int e^{-\left(-\sqrt{c^{2}-c_{*}^{2}} t-y\right)^{2} /(4 t)} S_{0}(y) d y \\
=\frac{1}{\sqrt{4 \pi}} \int e^{-\left(\sqrt{c^{2}-c_{*}^{2}} \sqrt{t}+z\right)^{2} / 4} S_{0}(z \sqrt{t}) d z \\
=\frac{1}{\sqrt{4 \pi}} \int_{-(1+\eta) \sqrt{c^{2}-c_{*}^{2}} \sqrt{t}}^{-(1-\eta) \sqrt{c^{2}-c_{*}^{2}} \sqrt{t}} e^{-\left(\sqrt{c^{2}-c_{*}^{2}} \sqrt{t}+z\right)^{2} / 4} S_{0}(z \sqrt{t}) d z \\
+\frac{1}{\sqrt{4 \pi}} \int_{\left|z+\sqrt{c^{2}-c_{*}^{2}} \sqrt{t}\right| \geq \eta \sqrt{c^{2}-c_{*}^{2}} \sqrt{t}} e^{-\left(\sqrt{c^{2}-c_{*}^{2}} \sqrt{t}+z\right)^{2} / 4} S_{0}(z \sqrt{t}) d z .
\end{gathered}
$$

The second integral goes exponentially fast to 0 , since

$$
\begin{aligned}
& \left|\frac{1}{\sqrt{4 \pi}} \int_{\left|z+\sqrt{c^{2}-c_{*}^{2}} \sqrt{t}\right| \geq \eta \sqrt{c^{2}-c_{*}^{2}} \sqrt{t}} e^{-\left(\sqrt{c^{2}-c_{*}^{2}} \sqrt{t}+z\right)^{2} / 4} S_{0}(z \sqrt{t}) d z\right| \\
& \leq 2\left\|S_{0}\right\|_{\infty} \int_{\eta \sqrt{c^{2}-c_{*}^{2}} \sqrt{t}}^{+\infty} e^{-x^{2} / 4} d x=\left\|S_{0}\right\|_{\infty} \int_{\eta^{2}\left(c^{2}-c_{*}^{2}\right) t} e^{-s / 4} \frac{d s}{\sqrt{s}} \\
& =\left\|S_{0}\right\|_{\infty} O\left(e^{-\eta^{2}\left(c^{2}-c_{*}^{2}\right) t / 4}\right) .
\end{aligned}
$$

Concerning the first integral, we use the fact that $S_{0}$ oscillates slowly:

$$
\begin{aligned}
& \frac{1}{\sqrt{4 \pi}} \int_{-(1+\eta) \sqrt{c^{2}-c_{*}^{2}} \sqrt{t}}^{-(1-\eta) \sqrt{c^{2}-c_{*}^{2}} \sqrt{t}} e^{-\left(\sqrt{c^{2}-c_{*}^{2}} \sqrt{t}+z\right)^{2} / 4} S_{0}(z \sqrt{t}) d z \\
& =\frac{1}{\sqrt{4 \pi}} \int_{-(1+\eta) \sqrt{c^{2}-c_{*}^{2}} \sqrt{t}}^{-(1-\eta) \sqrt{c^{2}-c_{*}^{2}} \sqrt{t}} e^{-\left(\sqrt{c^{2}-c_{*}^{2}} \sqrt{t}+z\right)^{2} / 4}\left(S_{0}(z \sqrt{t})-S_{0}\left(-\sqrt{c^{2}-c_{*}^{2}} t\right)\right) d z \\
& \quad+\frac{1}{\sqrt{4 \pi}} \int_{-(1+\eta) \sqrt{c^{2}-c_{*}^{2}} \sqrt{t}}^{-(1-\eta) \sqrt{c^{2}-c_{*}^{2}} \sqrt{t}} e^{-\left(\sqrt{c^{2}-c_{*}^{2}} \sqrt{t}+z\right)^{2} / 4} S_{0}\left(-\sqrt{c^{2}-c_{*}^{2}} t\right) d z
\end{aligned}
$$

since for $z \in\left(-(1+\eta) \sqrt{c^{2}-c_{*}^{2}} \sqrt{t},-(1-\eta) \sqrt{c^{2}-c_{*}^{2}} \sqrt{t}\right)$, we have

$$
1-\eta \leq \frac{z \sqrt{t}}{-\sqrt{c^{2}-c_{*}^{2}} t} \leq 1+\eta
$$

we obtain that

$$
\left|S_{0}(z \sqrt{t})-S_{0}\left(-\sqrt{c^{2}-c_{*}^{2}} t\right)\right| \leq \varepsilon
$$

hence

$$
\begin{aligned}
& \mid \frac{1}{\sqrt{4 \pi}} \int_{-(1+\eta) \sqrt{c^{2}-c_{*}^{2}} \sqrt{t}}^{-(1-\eta) \sqrt{c^{2}-c_{*}^{2}} \sqrt{t}} e^{-\left(\sqrt{c^{2}-c_{*}^{2}} \sqrt{t}+z\right)^{2} / 4}\left(S_{0}(z \sqrt{t})\right.\left.-S_{0}\left(-\sqrt{c^{2}-c_{*}^{2}} t\right)\right) d z \mid \\
& \leq \varepsilon \frac{1}{\sqrt{4 \pi}} \int_{\mathbb{R}} e^{-x^{2} / 4} d x
\end{aligned}
$$


Finally,

$$
\begin{aligned}
& \frac{1}{\sqrt{4 p i}} \int_{-(1+\eta) \sqrt{c^{2}-c_{*}^{2}} \sqrt{t}}^{-(1-\eta) \sqrt{c^{2}-c_{*}^{2}} \sqrt{t}} e^{-\left(\sqrt{c^{2}-c_{*}^{2}} \sqrt{t}+z\right)^{2} / 4} S_{0}\left(-\sqrt{c^{2}-c_{*}^{2}} t\right) d z \\
& =S_{0}\left(-\sqrt{c^{2}-c_{*}^{2}} t\right) \frac{1}{\sqrt{4 \pi}} \int_{-(1+\eta) \sqrt{c^{2}-c_{*}^{2}} \sqrt{t}}^{-(1-\eta) \sqrt{c^{2}-c_{*}^{2}} \sqrt{t}} e^{-\left(\sqrt{c^{2}-c_{*}^{2}} \sqrt{t}+z\right)^{2} / 4} d z \\
& =S_{0}\left(-\sqrt{c^{2}-c_{*}^{2}} t\right) \frac{1}{\sqrt{4 \pi}} \int_{-\eta \sqrt{c^{2}-c_{*}^{2}} \sqrt{t}}^{\eta \sqrt{c^{2}-c_{*}^{2}} \sqrt{t}} e^{-x^{2} / 4} d x \\
& =S_{0}\left(-\sqrt{c^{2}-c_{*}^{2}} t\right) \frac{1}{\sqrt{4 \pi}}\left(\int_{\mathbb{R}} e^{-x^{2} / 4} d x-2 \int_{\eta \sqrt{c^{2}-c_{*}^{2}} \sqrt{t}}^{+\infty} e^{-x^{2} / 4} d x\right) \\
& =S_{0}\left(-\sqrt{c^{2}-c_{*}^{2}} t\right)+O\left(\left\|S_{0}\right\|_{\infty} e^{-\eta^{2}\left(c^{2}-c_{*}^{2}\right) t / 4}\right) .
\end{aligned}
$$

Hence it follows from (2.14)-(2.17) that

$$
S\left(t,-\sqrt{c^{2}-c_{*}^{2}} t\right)=S_{0}\left(-\sqrt{c^{2}-c_{*}^{2}} t\right)+o(1),
$$

which proves (2.13). Hence $S\left(t,-\sqrt{c^{2}-c_{*}^{2}} t\right)$ oscillates between $\liminf _{x \rightarrow-\infty} S_{0}$ and $\lim \sup _{x \rightarrow-\infty} S_{0}$. The same oscillation property holds for

$$
m(t, 0)=\frac{1}{r_{-}(c)} \ln S\left(t,-\sqrt{c^{2}-c_{*}^{2}} t\right) .
$$

Since the solution $u$ converges uniformly to the approximate solution, it cannot converge to any translate of the travelling wave.

2.6.3. The typical case where the initial shift converges to some limit at $-\infty$. Now we assume that the initial shift $m_{0}$ has some limit $m_{0}(-\infty)$ at $-\infty$. We are going to prove the convergence of the solution $u$ of (1.2) to the travelling wave $\phi_{c}\left(x+c t+m_{0}(-\infty)\right)$. Thanks to Theorem 1.1, we only have to study the difference between the approximate solution $u^{a p p}(t, x)$ and $\phi_{c}\left(x+c t+m_{0}(-\infty)\right)$. Consider

$$
\bar{m}_{0}(x):=\sup _{y \leq x}\left|m_{0}(y)-m_{0}(-\infty)\right| .
$$

The function $\bar{m}_{0}$ is nondecreasing and $\bar{m}_{0}(x) \rightarrow 0$ as $x \rightarrow-\infty$. Then we are going to prove the following.

Lemma 2.9. There is some $\beta>0$ and $C>0$ such that

$\sup _{x \in \mathbb{R}}\left|u^{a p p}(t, x)-\phi_{c}\left(x+c t+m_{0}(-\infty)\right)\right| \leq C \bar{m}_{0}\left(-\frac{\sqrt{c^{2}-c_{*}^{2}}}{4} t\right)+C\left\|\bar{m}_{0}\right\|_{\infty} e^{-\beta \sqrt{c^{2}-c_{*}^{2}} t}$.

Moreover, this estimate is optimal in the following sense: assume, additionally, that $m_{0}$ is increasing in a neighborhood of $-\infty$; then

$$
\sup _{x \in \mathbb{R}}\left|u^{a p p}(t, x)-\phi_{c}\left(x+c t+m_{0}(-\infty)\right)\right| \geq C\left(m_{0}\left(-\sqrt{c^{2}-c_{*}^{2}} t\right)-m_{0}(-\infty)\right) .
$$

Remark 2.10. Lemma 2.9 gives an upper bound and a lower bound of the difference between the approximate solution $u^{a p p}$ and the travelling wave $\phi_{c}\left(\cdot+m_{0}(-\infty)\right)$ : if $m_{0}$ goes exponentially fast (or faster) to $m_{0}(-\infty)$, the difference $u^{a p p}(t, x)-$ $\phi_{c}\left(x+c t+m_{0}(-\infty)\right.$ goes exponentially fast in time to 0 . If $m_{0}$ goes algebraically fast to $m_{0}(-\infty)$ (as $1 /|x|^{\gamma}$, with some $\gamma>0$ ), the same occurs for the difference $u^{a p p}(t, x)-\phi_{c}\left(x+c t+m_{0}(-\infty)\right)$, at the same order, and not faster. Coupled 
with Theorem 1.1 we obtain a first result of convergence of the solution $u$ of the Cauchy problem (1.2) to the travelling wave $\phi_{c}\left(x+c t+m_{0}(-\infty)\right)$, with a rate of convergence that depends on the rate of convergence of $m_{0}$ to its limit. This extends Theorem 0.1, since the initial condition does not have to go exponentially fast to the travelling wave at $-\infty$. We will give a sharp convergence result in the following subsection, in particular to obtain exponential convergence when $m_{0}$ goes exponentially fast to its limit.

Proof of Lemma 2.9. First we note that for all $t>0, m(t, \xi) \rightarrow m_{0}(-\infty)$ as $t \rightarrow$ $+\infty$ : indeed, using the formula

$$
s(t, \xi)=S\left(t, \xi-\sqrt{c^{2}-c_{*}^{2}} t\right)=\frac{1}{\sqrt{\pi}} \int_{\mathbb{R}} e^{-\sigma^{2}} S_{0}\left(\xi+2 \sigma \sqrt{t}-\sqrt{c^{2}-c_{*}^{2}} t\right) d \sigma,
$$

we obtain that $s(t, \xi) \rightarrow e^{t_{-}(c) m_{0}(-\infty)}$ as $t \rightarrow+\infty$ (using the Lebesgue convergence theorem), and then $m(t, \xi) \rightarrow m_{0}(-\infty)$ as $t \rightarrow+\infty$. (However the convergence is nonuniform in $\xi$.)

Now we are interested in the difference $\tilde{u}^{a p p}(t, \xi)-\phi_{c}\left(\xi+m_{0}(-\infty)\right)$. Since by the stable manifold theorem we know that $\phi_{c}^{\prime}$ goes exponentially fast to 0 at $\pm \infty$, there exists some $\alpha>0$ such that

$$
\begin{aligned}
\left|\tilde{u}^{a p p}(t, \xi)-\phi_{c}\left(\xi+m_{0}(-\infty)\right)\right|=\mid \phi_{c}(\xi+m(t, \xi)) & -\phi_{c}\left(\xi+m_{0}(-\infty)\right) \mid \\
& \leq C e^{-\alpha|\xi|}\left|m(t, \xi)-m_{0}(-\infty)\right| .
\end{aligned}
$$

Of course

$$
\begin{aligned}
m(t, \xi)-m_{0}(-\infty) & =\frac{1}{r_{-}(c)} \ln S\left(t, \xi-\sqrt{c^{2}-c_{*}^{2}} t\right)-m_{0}(-\infty) \\
& =\frac{1}{r_{-}(c)} \ln \frac{S\left(t, \xi-\sqrt{c^{2}-c_{*}^{2}} t\right)}{e^{r_{-}(c) m_{0}(-\infty)}}
\end{aligned}
$$

hence

$$
\left|m(t, \xi)-m_{0}(-\infty)\right| \leq C\left|S\left(t, \xi-\sqrt{c^{2}-c_{*}^{2}} t\right)-e^{r_{-}(c) m_{0}(-\infty)}\right| .
$$

But

$$
\begin{aligned}
& S\left(t, \xi-\sqrt{c^{2}-c_{*}^{2}} t\right)-e^{r_{-}(c) m_{0}(-\infty)} \\
& =\frac{1}{\sqrt{\pi}} \int_{\mathbb{R}} e^{-\sigma^{2}} S_{0}\left(\xi+2 \sigma \sqrt{t}-\sqrt{c^{2}-c_{*}^{2}} t\right) d \sigma-e^{r_{-}(c) m_{0}(-\infty)} \\
& =\frac{1}{\sqrt{\pi}} \int_{\mathbb{R}} e^{-\sigma^{2}}\left(e^{r_{-}(c) m_{0}\left(\xi+2 \sigma \sqrt{t}-\sqrt{c^{2}-c_{*}^{2}} t\right)}-e^{r_{-}(c) m_{0}(-\infty)}\right) d \sigma \\
& \leq C \int_{\mathbb{R}} e^{-\sigma^{2}}\left|m_{0}\left(\xi+2 \sigma \sqrt{t}-\sqrt{c^{2}-c_{*}^{2}} t\right)-m_{0}(-\infty)\right| d \sigma \\
& \leq C \int_{\mathbb{R}} e^{-\sigma^{2}} \bar{m}_{0}\left(\xi+2 \sigma \sqrt{t}-\sqrt{c^{2}-c_{*}^{2}} t\right) d \sigma ;
\end{aligned}
$$

hence

$$
\left|\tilde{u}^{a p p}(t, \xi)-\phi_{c}\left(\xi+m_{0}(-\infty)\right)\right| \leq C e^{-\alpha|\xi|} \int_{\mathbb{R}} e^{-\sigma^{2}} \bar{m}_{0}\left(\xi+2 \sigma \sqrt{t}-\sqrt{c^{2}-c_{*}^{2}} t\right) d \sigma .
$$


It remains to estimate, uniformly in $\xi$, this last term. This can be done, separating the study into three cases:

- If $\xi \leq 0$, then we have

$$
\begin{gathered}
e^{-\alpha|\xi|} \int_{\mathbb{R}} e^{-\sigma^{2}} \bar{m}_{0}\left(\xi+2 \sigma \sqrt{t}-\sqrt{c^{2}-c_{*}^{2}} t\right) d \sigma \\
\leq \int_{-\infty}^{\sqrt{c^{2}-c_{*}^{2}} \sqrt{t} / 4} e^{-\sigma^{2}} \bar{m}_{0}\left(\xi+2 \sigma \sqrt{t}-\sqrt{c^{2}-c_{*}^{2}} t\right) d \sigma \\
\quad+\int_{\sqrt{c^{2}-c_{*}^{2}} \sqrt{t} / 4}^{+\infty} e^{-\sigma^{2}} \bar{m}_{0}\left(\xi+2 \sigma \sqrt{t}-\sqrt{c^{2}-c_{*}^{2}} t\right) d \sigma \\
\leq \bar{m}_{0}\left(-\frac{\sqrt{c^{2}-c_{*}^{2}}}{2} t\right) \int_{-\infty}^{\sqrt{c^{2}-c_{*}^{2}} \sqrt{t} / 4} e^{-\sigma^{2}} d \sigma+\left\|\bar{m}_{0}\right\|_{\infty} \int_{\sqrt{c^{2}-c_{*}^{2}} \sqrt{t} / 4}^{+\infty} e^{-\sigma^{2}} d \sigma \\
\leq \sqrt{\pi} \bar{m}_{0}\left(-\frac{\sqrt{c^{2}-c_{*}^{2}}}{2} t\right)+C\left\|\bar{m}_{0}\right\|_{\infty} e^{-\left(c^{2}-c_{*}^{2}\right) t / 16}
\end{gathered}
$$

- If $\xi \geq \frac{\sqrt{c^{2}-c_{*}^{2}} t}{2}$, then we have

$$
e^{-\alpha|\xi|} \int_{\mathbb{R}} e^{-\sigma^{2}} \bar{m}_{0}\left(\xi+2 \sigma \sqrt{t}-\sqrt{c^{2}-c_{*}^{2}} t\right) d \sigma \leq \sqrt{\pi} e^{-\alpha \sqrt{c^{2}-c_{*}^{2}} t / 2}\left\|\bar{m}_{0}\right\|_{\infty}
$$

- If $\xi \in\left(0, \frac{\sqrt{c^{2}-c_{*}^{2}} t}{2}\right)$, then we have

$$
\begin{gathered}
e^{-\alpha|\xi|} \int_{\mathbb{R}} e^{-\sigma^{2}} \bar{m}_{0}\left(\xi+2 \sigma \sqrt{t}-\sqrt{c^{2}-c_{*}^{2}} t\right) d \sigma \\
\leq \int_{-\infty}^{\sqrt{c^{2}-c_{*}^{2}} \sqrt{t} / 8} e^{-\sigma^{2}} \bar{m}_{0}\left(2 \sigma \sqrt{t}-\frac{\sqrt{c^{2}-c_{*}^{2}}}{2} t\right) d \sigma \\
+\int_{\sqrt{c^{2}-c_{*}^{2}} \sqrt{t} / 8}^{+\infty} e^{-\sigma^{2}} \bar{m}_{0}\left(2 \sigma \sqrt{t}-\frac{\sqrt{c^{2}-c_{*}^{2}}}{2} t\right) d \sigma \\
\leq \sqrt{\pi} \bar{m}_{0}\left(-\frac{\sqrt{c^{2}-c_{*}^{2}}}{4} t\right)+C\left\|\bar{m}_{0}\right\|_{\infty} e^{-\left(c^{2}-c_{*}^{2}\right) t / 64}
\end{gathered}
$$

and these three estimates and (2.21) lead us to (2.19).

To obtain (2.20), we want to bound from below the difference $\| \tilde{u}^{a p p}(t, \cdot)-$ $\phi_{c}\left(\cdot+m_{0}(-\infty)\right) \|_{\infty}$; for this, we remark that, for $\xi=0$, there is some $\alpha>0$ such that

$$
\begin{aligned}
& \left|u^{a p p}(t, 0)-\phi_{c}\left(m_{0}(-\infty)\right)\right|=\left|\phi_{c}(m(t, 0))-\phi_{c}\left(m_{0}(-\infty)\right) \geq \alpha\right| m(t, 0)-m_{0}(-\infty) \mid \\
= & \frac{\alpha}{r_{-}(c)} \ln \frac{S\left(t,-\sqrt{c^{2}-c_{*}^{2}} t\right)}{e^{r_{-}(c) m_{0}(-\infty)}}=\frac{\alpha}{r_{-}(c)} \ln \left(1+\frac{S\left(t,-\sqrt{c^{2}-c_{*}^{2}} t\right)-e^{r_{-}(c) m_{0}(-\infty)}}{e^{r_{-}(c) m_{0}(-\infty)}}\right) ;
\end{aligned}
$$


hence, for $t$ large enough, we have

$$
\begin{aligned}
& \left|u^{a p p}(t, 0)-\phi_{c}\left(m_{0}(-\infty)\right)\right| \geq \frac{\alpha}{2 r_{-}(c)} \frac{S\left(t,-\sqrt{c^{2}-c_{*}^{2}} t\right)-e^{r_{-}(c) m_{0}(-\infty)}}{e^{r_{-}(c) m_{0}(-\infty)}} \\
& \geq \frac{\alpha}{2 r_{-}(c) e^{r_{-}(c) m_{0}(-\infty)}} \frac{1}{\sqrt{\pi}} \int_{\mathbb{R}} e^{-\sigma^{2}}\left(e^{r_{-}(c) m_{0}\left(2 \sigma \sqrt{t}-\sqrt{c^{2}-c_{*}^{2}} t\right)}-e^{r_{-}(c) m_{0}(-\infty)}\right) d \sigma \\
& \geq \frac{\alpha}{2 r_{-}(c) e^{r_{-}(c) m_{0}(-\infty)}} \frac{1}{\sqrt{\pi}} \int_{0}^{1} e^{-\sigma^{2}}\left(e^{r_{-}(c) m_{0}\left(2 \sigma \sqrt{t}-\sqrt{c^{2}-c_{*}^{2}} t\right)}-e^{r_{-}(c) m_{0}(-\infty)}\right) d \sigma \\
& \geq C \inf _{\sigma \in[0,1]}\left|m_{0}\left(2 \sigma \sqrt{t}-\sqrt{c^{2}-c_{*}^{2}} t\right)-m_{0}(-\infty)\right| \\
& \geq C\left(m_{0}\left(-\sqrt{c^{2}-c_{*}^{2}} t\right)-m_{0}(-\infty)\right) .
\end{aligned}
$$

This gives the bound from below (2.20) and concludes the proof of Lemma 2.9.

2.6.4. Sharp estimate of the convergence of the solution $u$ to the travelling wave when the initial shift has a limit at $-\infty$. The goal of this subsection, the last on the study of the simple 1D problem, is to give a sharp estimate of the convergence of the solution $u$ of the Cauchy problem (1.2) to the travelling wave $\phi_{c}\left(x+c t+m_{0}(-\infty)\right)$ when the initial shift $m_{0}$ has a limit $m_{0}(-\infty)$ at $-\infty$. We keep the same notation as in the previous subsection. Since the function $\bar{m}_{0}$ is nondecreasing, and goes to 0 at $-\infty$, there exists some nondecreasing function $\bar{n}_{0}$ such that $\bar{m}_{0} \leq \bar{n}_{0}$ and such that there is some constant $C_{0}$ such that

$$
\bar{n}_{0}^{\prime} \leq C_{0} \bar{n}_{0} .
$$

The function $\bar{n}_{0}$ identically equal to $\left\|\bar{m}_{0}\right\|_{\infty}$ satisfies this property, but in the typical cases there exists some $\bar{n}_{0}$ that goes to zero at $-\infty$ with the same rate of decay as $\bar{m}_{0}$. Then we are going to prove the following.

Proposition 2.11. There is some $\beta>0$ and $C>0$ such that

$$
\sup _{x \in \mathbb{R}}\left|u(t, x)-u^{a p p}(t, x)\right| \leq C \frac{\bar{n}_{0}(-\beta t)}{\sqrt{1+\beta t}}+C\left\|\bar{m}_{0}\right\|_{\infty} e^{-\beta \sqrt{c^{2}-c_{*}^{2}} t} .
$$

Remark 2.12. Proposition 2.11 gives an upper bound for the difference between the solution $u$ and the approximate solution $u^{a p p}$ : the convergence is at least as $t^{-1 / 2}$, as proved in Theorem 1.1, but the rate of convergence is in fact better:

- If $m_{0}$ goes exponentially fast (or faster) to $m_{0}(-\infty)$, the difference $u-u^{a p p}$ goes exponentially fast in time to 0 ; thanks to Lemma 2.9 the same occurs for the difference between the solution and the travelling wave $\phi_{c}\left(x+c t+m_{0}(-\infty)\right)$, as already proved in Theorem 0.1.

- If $m_{0}$ goes algebraically fast to $m_{0}(-\infty)$ (as $1 /|x|^{\gamma}$, with some $\left.\gamma>0\right)$, then the difference $u-u^{a p p}$ is of order $t^{-(\gamma+1 / 2)}$; coupled with Lemma 2.9, we obtain a sharp description of what happens: the solution is close to the approximate solution, at the order $t^{-(\gamma+1 / 2)}$, and the approximate solution is close to the travelling wave $\phi_{c}\left(x+c t+m_{0}(-\infty)\right.$ ), at the order $t^{-\gamma}$ (and in some cases not better).

Proof of Lemma 2.11. The proof combines the arguments used in the proof of Lemma 2.9 with the techniques used in subsection 2.5.2. First, from the formula 
(2.6) of $S$, we note that

$S_{\xi}\left(t, \xi-\sqrt{c^{2}-c_{*}^{2}} t\right)=\frac{-1}{\sqrt{\pi t}} \int_{\mathbb{R}} \sigma e^{-\sigma^{2}}\left(S_{0}\left(\xi+2 \sigma \sqrt{t}-\sqrt{c^{2}-c_{*}^{2}} t\right)-e^{r_{-}(c) m_{0}(-\infty)}\right) d \sigma ;$

hence

$$
\left|S_{\xi}\left(t, \xi-\sqrt{c^{2}-c_{*}^{2}} t\right)\right| \leq \frac{C}{\sqrt{t}} \int_{\mathbb{R}}|\sigma| e^{-\sigma^{2}} \bar{m}_{0}\left(\xi+2 \sigma \sqrt{t}-\sqrt{c^{2}-c_{*}^{2}} t\right) d \sigma .
$$

Next, we have already noted that the function $g(t, \xi)$ that is defined in (2.2) satisfies:

$$
g(t, \xi)=O\left(e^{\left.r_{-}(c)+\delta\right) \xi}\right) \text { as } \xi \rightarrow-\infty, \quad g(t, \xi)=O\left(e^{-\alpha \xi}\right) \text { as } \xi \rightarrow+\infty,
$$

with some $\alpha>0$. Then, arguing as in the proof of Lemma 2.9. we see that

$$
\left|N L_{c}\left[\tilde{u}^{a p p}\right]\right| \leq\left\{\begin{array}{l}
\left(C \frac{\bar{m}_{0}\left(-\frac{\sqrt{c^{2}-c_{*}^{2}}}{4} t\right)}{\sqrt{t}}+C\left\|\bar{m}_{0}\right\|_{\infty} e^{-\beta \sqrt{c^{2}-c_{*}^{2}} t}\right) e^{\left.r_{-}(c)+\delta\right) \xi} \text { for } \xi \leq 0, \\
\left(C \frac{\bar{m}_{0}\left(-\frac{\sqrt{c^{2}-c_{*}^{2}}}{4}\right)}{\sqrt{t}}+C\left\|\bar{m}_{0}\right\|_{\infty} e^{-\beta \sqrt{c^{2}-c_{*}^{2}} t}\right) e^{-\alpha \xi / 2} \text { for } \xi \geq 0 .
\end{array}\right.
$$

Of course Claim 2.2 remains valid, and we have $\left|\tilde{u}(t, \xi)-\tilde{u}^{a p p}(t, \xi)\right| \leq \tilde{w}(t, \xi)$. But thanks to (2.23), we are in a position to improve our estimate of Claim 2.3. The same proof leads to the following: for all $\xi_{0}$, there exists some $C$ such that

$$
\sup _{\xi \leq \xi_{0}} \tilde{w}(t, \xi) \leq C \frac{\bar{m}_{0}\left(-\frac{\sqrt{c^{2}-c_{*}^{2}}}{8} t\right)}{\sqrt{t}}+C\left\|\bar{m}_{0}\right\|_{\infty} e^{-\beta \sqrt{c^{2}-c_{*}^{2}} t} .
$$

At last, arguing as in Claim 2.4, we obtain (2.22).

\section{The general model (1.1): Proof of Theorem 1.3}

We use the same notation as in Section 2 i.e., we set $\tilde{u}(t, \xi, y):=u(t, \xi-c t, y)$.

3.1. Pulsating waves with sharp asymptotic behavior. The behavior of a pulsating wave at $-\infty$ is now well known: similarly to the simple ODE case there are, for $c>c_{*}$, two characteristic exponents $r_{ \pm}(c)$, corresponding to exponential solutions of the linearized equation at $-\infty$, and $\phi_{c}$ has a nontrivial component on the slowest exponential. We will need something a bit more precise, i.e. a quantitative estimate on the remainder, so it is worth recalling the main results of the theory.

For commodity let us denote by $X=(x, y)$ the generic element of $\mathbb{T}^{N}$. Consider equation (1.1), linearized at 0 :

$$
v_{t}-\operatorname{div}(A D v)+B \cdot D v-f_{u}(X, 0) v=0 .
$$

An exponential solution to (3.1) is a solution of the form

$$
v(t, x, y)=e^{\lambda(x+c t)} \psi(X), \quad X \in \mathbb{T}^{N},
$$

where $\lambda>0$ is called a characteristic exponent. Denoting $e_{1}=(1,0, \ldots, 0)$ and $B_{1}:=B \cdot e_{1}$, the first component of $B$, we see that the function $\psi(X)$ has to solve

$$
L(\lambda) \psi=-c \lambda \psi, \quad X \in \mathbb{T}^{N},
$$

where

$$
\begin{aligned}
L(\lambda) \psi:= & -\operatorname{div}(A D \psi)+\left(B-2 \lambda A e_{1}\right) \cdot D \psi \\
& +\left(-\lambda^{2} A e_{1} \cdot e_{1}-\lambda \operatorname{div}\left(A e_{1}\right)+\lambda B_{1}-f_{u}(X, 0)\right) \psi
\end{aligned}
$$


Because $\phi_{c}$ has to be positive, $\psi$ has to be positive, and so, if we denote by $\mu_{1}(\lambda)$ the first eigenvalue of $L(\lambda)$, problem (3.2) amounts to solving

$$
c=-\frac{\mu_{1}(\lambda)}{\lambda}
$$

Because of Krein-Rutman's Theorem, $\mu_{1}(\lambda)$ is a simple, nondegenerate eigenvalue for $L(\lambda)$ and Kato-Rellich's Theorem implies the existence of an analytic extension of $\mu_{1}$ in a complex domain containing the right half-line. In particular, $\lambda \mapsto \mu_{1}(\lambda)$ is $C^{1}$. More special features of the function $\mu_{1}$ are summarized in the following.

Theorem 3.1 (Berestycki-Hamel [3]). The function $\mu_{1}$ is concave, and

$$
\inf _{\lambda>0}\left(-\frac{\mu_{1}(\lambda)}{\lambda}\right)>0
$$

Then, defining

$$
c_{*}:=\inf _{\lambda>0}\left(-\frac{\mu_{1}(\lambda)}{\lambda}\right),
$$

equation (3.4) has solutions if and only if $c \geq c_{*}$; moreover, when $c>c_{*}$, there are two solutions $0<r_{-}(c)<r_{+}(c)$.

This implies, in particular:

$$
V_{*}(c):=c+\frac{d \mu_{1}}{d \lambda}\left(r_{-}(c)\right)>0 .
$$

Equation (3.6) will be useful to us at several places. Let $\psi_{\lambda}$ be the unique principal eigenfunction of $L(\lambda)$ such that

$$
\psi_{\lambda}>0 \quad \text { and } \quad \int_{\mathbb{T}^{N}} \psi_{\lambda}^{2}=1 .
$$

We will also denote

$$
r_{c}:=r_{-}(c), \quad \psi_{r_{c}}:=\psi_{r_{-}(c)} .
$$

The additional information that we need for $\phi_{c}$ is

Theorem 3.2. There is $\delta>0$ such that the wave $\tilde{\phi}_{c}$ has the following asymptotic behavior as $\xi \rightarrow-\infty$ :

$$
\tilde{\phi}_{c}(t, \xi, y)=e^{r_{-}(c) \xi} \psi_{r_{c}}(\xi-c t, y)\left(1+O\left(e^{\delta \xi}\right)\right) .
$$

The equivalence $\tilde{\phi}_{c}(t, \xi, y) \sim e^{r_{-}(c) \xi} \psi_{r_{c}}(\xi-c t, y)$ was proved by Hamel [12]. What we need is the exponential estimate on the difference between $\tilde{\phi}_{c}$ and its equivalent. We could elaborate on 12 to get hold of it; it will in fact be quicker to prove an existence result for $\phi_{c}$ with the right behavior and invoke a uniqueness result.

Proposition 3.3. There exists $\delta \in\left(0, \inf \left(r_{-}(c), r_{+}(c)-r_{-}(c)\right)\right)$ such that there is a pulsating wave $\phi_{c}$ solution to (1.1) with speed $c$ satisfying the asymptotic expansion (3.7). Moreover, $\phi_{c}$ is increasing in its first variable: $\partial_{t} \phi_{c}>0$ (or equivalently $\left.\partial_{t} \tilde{\phi}_{c}+c \partial_{\xi} \tilde{\phi}_{c}>0\right)$.

The proof of Proposition 3.3 follows from a fixed point argument. Before proving it, we need a preliminary result, which will play the role of Lemma 2.1 in Section 2. 
3.1.1. Preliminary result: Exponential decay in time of solutions decaying suffciently fast in space. Given $\delta \in\left(0, r_{+}(c)-r_{-}(c)\right)$, let $Y_{\delta}$ be the space of all continuous functions $\tilde{u}$ on $\mathbb{R} \times \mathbb{T}^{N-1}$ such that

$$
\tilde{u}(\xi, y) e^{-\left(r_{-}(c)+\delta\right) \xi} \text { is a bounded uniformly continuous function, }
$$

endowed with the natural norm $\|\tilde{u}\|_{Y_{\delta}}:=\sup _{(\xi, y) \in \mathbb{R} \times \mathbb{T}^{N-1}}|\tilde{u}(\xi, y)| e^{-\left(r_{-}(c)+\delta\right) \xi}$.

Lemma 3.4. Consider the equation

$$
\tilde{v}_{t}-\operatorname{div}(A(\xi-c t, y) D \tilde{v})+\left(B(\xi-c t, y)+c e_{1}\right) \cdot D \tilde{v}-f_{u}(\xi-c t, y, 0) \tilde{v}=0 .
$$

Then, given $\delta \in\left(0, r_{+}(c)-r_{-}(c)\right)$, there is $C_{\delta} \geq 1$ and $\omega_{\delta}>0$ such that, if $\tilde{v}(0,):.=\tilde{v}_{0}$ is in $Y_{\delta}$, then the solution $\tilde{v}$ of (3.8) emerging from $\tilde{v}_{0}$ satisfies: for all $t>0, \tilde{v}(t, \cdot, \cdot) \in Y_{\delta}$, and

$$
\|\tilde{v}(t, \cdot, \cdot)\|_{Y_{\delta}} \leq C_{\delta} e^{-\omega_{\delta} t}\left\|\tilde{v}_{0}\right\|_{Y_{\delta}}
$$

Proof of Lemma 3.4. The proof follows from the construction of a suitable positive supersolution to (3.8), exponentially decaying in time. The construction of this positive supersolution rests on the properties of the first eigenvalue of the elliptic operator $L_{\lambda}$ (defined in (3.3)), which we recalled in Theorem 3.1.

First, given $\lambda>0$ and $\omega \geq 0$, let us consider

$$
\tilde{V}^{(\lambda, \omega)}(t, \xi, y):=\psi_{\lambda}(\xi-c t, y) e^{\lambda \xi-\omega t} .
$$

Then

$$
\begin{aligned}
\tilde{V}_{t}^{(\lambda, \omega)}-\operatorname{div}\left(A(\xi-c t, y) D \tilde{V}^{(\lambda, \omega)}\right)+\left(B(\xi-c t, y)+c e_{1}\right) \cdot D \tilde{V}^{(\lambda, \omega)} \\
-f_{u}(\xi-c t, y, 0) \tilde{V}^{(\lambda, \omega)}=\left(\mu_{1}(\lambda)+c \lambda-\omega\right) \tilde{V}^{(\lambda, \omega)} .
\end{aligned}
$$

Since we want $\tilde{V}^{(\lambda, \omega)}$ to be positive and we want it to be a supersolution to (3.8), we need to find $\lambda$ and $\omega$ such that

$$
\mu_{1}(\lambda)+c \lambda-\omega \geq 0 .
$$

This can be done, noting that it follows from Theorem 3.1 that, given $\delta \in\left(0, r_{+}(c)-\right.$ $\left.r_{-}(c)\right)$, we have

Hence consider

$$
\mu_{1}\left(r_{-}(c)+\delta\right)+c\left(r_{-}(c)+\delta\right)>0
$$

$$
\lambda=\lambda_{\delta}:=r_{-}(c)+\delta \quad \text { and } \quad \omega=\omega_{\delta}:=\mu_{1}\left(r_{-}(c)+\delta\right)+c\left(r_{-}(c)+\delta\right) .
$$

Then we obtain that $\tilde{V}^{\delta}:=\tilde{V}^{\left(\lambda_{\delta}, \omega_{\delta}\right)}$ is a solution of (3.8):

$$
\begin{aligned}
\tilde{V}_{t}^{\delta}-\operatorname{div}\left(A(\xi-c t, y) D \tilde{V}^{\delta}\right)+(B(\xi-c t, y) & \left.+c e_{1}\right) \cdot D \tilde{V}^{\delta} \\
& -f_{u}(\xi-c t, y, 0) \tilde{V}^{\delta}=\left(\mu_{1}\left(\lambda_{\delta}\right)+c \lambda_{\delta}-\omega_{\delta}\right) \tilde{V}^{\delta}=0 .
\end{aligned}
$$

Now, since $\psi_{\lambda}$ is positive on the compact $\mathbb{T}^{N}$, denoting $\tilde{C}_{\delta}:=\frac{1}{\inf _{\mathbb{T}^{N}} \psi_{r_{-}(c)+\delta}}$, we have for all $\tilde{v}_{0} \in Y_{\delta}$,

$$
\left|\tilde{v}_{0}(\xi, y)\right| \leq \tilde{C}_{\delta} \psi_{r_{-}(c)+\delta}(\xi, y) e^{\left(r_{-}(c)+\delta\right) \xi}\left\|\tilde{v}_{0}\right\|_{Y_{\delta}}=\tilde{C}_{\delta}\left\|\tilde{v}_{0}\right\|_{Y_{\delta}} \tilde{V}(0, \xi, y) .
$$

Then, the weak maximum principle implies, for $(t, \xi, y) \in \mathbb{R}_{+} \times \mathbb{R} \times \mathbb{T}^{N-1}$ :

$$
|\tilde{v}(t, \xi, y)| \leq \tilde{C}_{\delta}\left\|\tilde{v}_{0}\right\|_{Y_{\delta}} \tilde{V}^{\delta}(t, \xi, y)=\tilde{C}_{\delta}\left\|\tilde{v}_{0}\right\|_{Y_{\delta}} \psi_{r_{-}(c)+\delta}(\xi-c t, y) e^{\left(r_{-}(c)+\delta\right) \xi-\omega_{\delta} t},
$$


which implies that $\tilde{v}(t, \cdot, \cdot) \in Y_{\delta}$ for all $t>0$, and that (3.9) is satisfied with $C_{\delta}=\frac{\sup _{\mathbb{T}^{N}} \psi_{r_{-}(c)+\delta}}{\inf _{\mathbb{T}^{N}} \psi_{r_{-}(c)+\delta}}$.

3.1.2. Application: Proof of Proposition 3.3. We want to prove that there is a pulsating wave solution of problem (1.1) that is a function $\phi_{c}$ satisfying (1.1) and the periodicity condition: $t \mapsto \phi_{c}(t, \xi-c t, y)$ is $\frac{1}{c}$-periodic. Translating this into the moving frame $(t, \xi=x+c t)$, the function $\tilde{\phi}_{c}(t, \xi, y):=\phi_{c}(t, \xi-c t, y)$ has to solve the problem

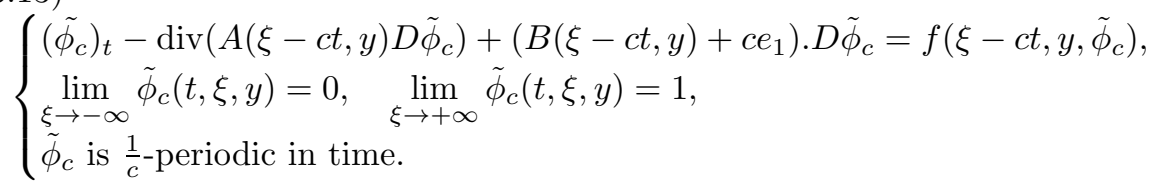

To prove the existence of such a $\frac{1}{c}$-periodic solution $\tilde{\phi}_{c}$ of this nonlinear problem, we are going to use a fixed point argument. An alternative method would be by a sub/supersolution argument, as for instance in [2, 15, or [21]. Our proof is divided into three steps:

- Step 1. We will prove that there is a solution to

$$
\left\{\begin{array}{l}
\left(\tilde{\phi}_{c}\right)_{t}-\operatorname{div}\left(A(\xi-c t, y) D \tilde{\phi}_{c}\right)+\left(B(\xi-c t, y)+c e_{1}\right) \cdot D \tilde{\phi}_{c}=f\left(\xi-c t, y, \tilde{\phi}_{c}\right), \\
\lim _{\xi \rightarrow-\infty} \tilde{\phi}_{c}(t, \xi, y)=0 \\
\tilde{\phi}_{c} \text { is } \frac{1}{c} \text {-periodic in time. }
\end{array}\right.
$$

- Step 2. We will prove that this $\tilde{\phi}_{c}$ satisfies the limit condition

$$
\lim _{\xi \rightarrow+\infty} \tilde{\phi}_{c}(t, \xi, y)=1 \text {. }
$$

- Step 3. We will prove that $\phi_{c}$ is increasing in its first variable: $\partial_{t} \phi_{c}>0$.

Step 1 (Existence of a periodic solution, using a fixed point argument). Our strategy is the following: considering the Cauchy problem

$$
\left\{\begin{array}{l}
\tilde{u}_{t}-\operatorname{div}(A(\xi-c t, y) D \tilde{u})+\left(B(\xi-c t, y)+c e_{1}\right) \cdot D \tilde{u}=f(\xi-c t, y, \tilde{u}) \\
\tilde{u}(0, \xi, y)=\tilde{u}_{0}
\end{array}\right.
$$

we want to find a suitable initial condition $\tilde{u}_{0}$ such that $\tilde{u}\left(\frac{1}{c}\right)=\tilde{u}_{0}$; then the periodicity assumptions on $A, B$ and $f$ will allow us to conclude that $\tilde{u}$ is $\frac{1}{c}$ periodic in time. To prove the existence of such an initial condition $\tilde{u}_{0}$, we are going to prove that the Poincaré map $\mathcal{T}_{1 / c}: \tilde{u}_{0} \mapsto \tilde{u}\left(\frac{1}{c}\right)$ is a contraction if we choose sufficiently well the space of initial conditions $\tilde{u}_{0}$.

First, we observe that the function

$$
\tilde{V}(t, \xi, y)=\tilde{V}^{\left(\lambda_{0}, \omega_{0}\right)}=\psi_{r_{-}(c)}(\xi-c t, y) e^{r_{-}(c) \xi}
$$


is a solution of (3.8), as noted in (3.12). This invites us to consider the following metric space: given $\delta \in\left(0, r_{+}(c)-r_{-}(c)\right)$, consider

$$
X_{\delta}:=\left\{\tilde{u}_{0} \in Y_{0}: \quad 0 \leq \tilde{u}_{0} \leq 1, \quad 0 \leq \tilde{u}_{0} \leq \tilde{V}(0), \tilde{V}(0)-\tilde{u}_{0} \in Y_{\delta}\right\}
$$

the distance between two elements of $X_{\delta}$ will be the $Y_{\delta}$-norm of the difference. We are going to prove the

Claim 3.5. There is a ball of $X_{\delta}$, centered at $\tilde{V}(0)$, that is stable under $\mathcal{T}_{1 / c}$; moreover, $\mathcal{T}_{1 / c}$ has a fixed point in this ball.

Indeed, first note that the weak maximum principle implies that $0 \leq \tilde{u} \leq 1$; next, the concavity of $f$ with respect to $u$ implies that

$$
\left\{\begin{array}{l}
\tilde{u}_{t}-\operatorname{div}(A(\xi-c t, y) D \tilde{u})+\left(B(\xi-c t, y)+c e_{1}\right) \cdot D \tilde{u}=f(\xi-c t, y, \tilde{u}) \\
\leq f_{u}(\xi-c t, y, 0) \tilde{u} \\
\tilde{V}_{t}-\operatorname{div}(A(\xi-c t, y) D \tilde{V})+\left(B(\xi-c t, y)+c e_{1}\right) \cdot D \tilde{V}=f_{u}(\xi-c t, y, 0) \tilde{V} \\
\tilde{u}(0) \leq \tilde{V}(0) .
\end{array}\right.
$$

Hence the weak maximum principle implies that $\tilde{u} \leq \tilde{V}$. Finally, the difference $\tilde{V}-\tilde{u}$ solves

$$
\begin{aligned}
(\tilde{V}-\tilde{u})_{t}-\operatorname{div}(A(\xi-c t, y) D(\tilde{V}-\tilde{u}))+\left(B(\xi-c t, y)+c e_{1}\right) \cdot D(\tilde{V}-\tilde{u}) \\
\quad-f_{u}(\xi-c t, y, 0)(\tilde{V}-\tilde{u})=f_{u}(\xi-c t, y, 0) \tilde{u}-f(\xi-c t, y, \tilde{u}) .
\end{aligned}
$$

Let us denote by $\tilde{g}(t, \xi, y)$ the right-hand member of this equality: Duhamel's formula tells us that

$$
(\tilde{V}-\tilde{u})(t)=T(t, 0)\left(\tilde{V}(0)-\tilde{u}_{0}\right)+\int_{0}^{t} T(t, s) \tilde{g}(s, \cdot, \cdot) d s,
$$

where $T(t, s) \tilde{v}_{0}$ is the solution at time $t$ of (3.8) starting from $\tilde{v}_{0}$ at time $s$. The following is true for the right-hand side of (3.16):

- first we note that, since $\tilde{V}(0)-\tilde{u}_{0} \in Y_{\delta}$, Lemma 3.4 gives that $T(t, 0)(\tilde{V}(0)-$ $\left.\tilde{u}_{0}\right) \in Y_{\delta}$ and

$$
\left\|T(t, 0)\left(\tilde{V}(0)-\tilde{u}_{0}\right)\right\|_{Y_{\delta}} \leq C_{\delta} e^{-\omega_{\delta} t}\left\|\tilde{V}(0)-\tilde{u}_{0}\right\|_{Y_{\delta}}
$$

- concerning the integral term, we have to make two remarks:

Claim 3.6. If additionally $\delta \in\left(0, r_{-}(c)\right)$, then for all $t>0, \tilde{g}(t, \cdot, \cdot) \in Y_{\delta}$.

Indeed, since $0 \leq \tilde{u} \leq \inf (1, \tilde{V})$, there is some $C>0$ independent of $\tilde{u}_{0}$ such that $\forall t \geq 0, \forall \xi \in \mathbb{R}, \forall y \in \mathbb{T}^{N-1},|\tilde{g}(t, \xi, y)|=\left|f(\xi-c t, y, \tilde{u})-f_{u}(\xi-c t, y, 0) \tilde{u}\right| \leq C e^{2 r_{-}(c) \xi} ;$ hence, if additionally $\delta \in\left(0, r_{-}(c)\right)$, there is some $C^{\prime}>0$ independent of $\tilde{u}_{0}$ such that

$$
\begin{aligned}
\forall t \geq 0, \forall \xi \in \mathbb{R}, \forall y \in \mathbb{T}^{N-1}, \quad|\tilde{g}(t, \xi, y)| & \leq C e^{\left(r_{-}(c)+\delta\right) \xi} \\
& \leq C^{\prime} \psi_{r_{-}(c)+\delta}(\xi-c t, y) e^{\left(r_{-}(c)+\delta\right) \xi},
\end{aligned}
$$

which establishes Claim 3.6 .

Claim 3.7.

$$
T(t, s)\left(\psi_{r_{-}(c)+\delta}(\xi-c s, y) e^{\left(r_{-}(c)+\delta\right) \xi}\right)=\psi_{r_{-}(c)+\delta}(\xi-c t, y) e^{\left(r_{-}(c)+\delta\right) \xi-\omega_{\delta}(t-s)} .
$$


Indeed, this follows from (3.12).

Using Claims 3.6 and 3.7 we easily derive from the weak maximum principle that

$$
\begin{aligned}
& \int_{0}^{t} T(t, s) \tilde{g}(s, \xi, y) d s \leq \int_{0}^{t} T(t, s)\left(C^{\prime} \psi_{r_{-}(c)+\delta}(\xi-c s, y) e^{\left(r_{-}(c)+\delta\right) \xi}\right) d s \\
= & C^{\prime} \int_{0}^{t} \psi_{r_{-}(c)+\delta}(\xi-c t, y) e^{\left(r_{-}(c)+\delta\right) \xi-\omega_{\delta}(t-s)} d s \leq \frac{C^{\prime}}{\omega_{\delta}} \psi_{r_{-}(c)+\delta}(\xi-c t, y) e^{\left(r_{-}(c)+\delta\right) \xi}
\end{aligned}
$$

hence $\int_{0}^{t} T(t, s) \tilde{g}(s, \cdot, \cdot) d s \in Y_{\delta}$, and there is some $C_{\delta}^{\prime}>0$ independent of $\tilde{u}_{0}$ such that

$$
\left\|\int_{0}^{t} T(t, s) \tilde{g}(s, \cdot, \cdot) d s\right\|_{Y_{\delta}} \leq C_{\delta}^{\prime} .
$$

Then Duhamel's formula (3.16) tells us that $(\tilde{V}-\tilde{u})\left(\frac{1}{c}\right) \in Y_{\delta}$. Since $\tilde{V}\left(\frac{1}{c}\right)=\tilde{V}(0)$, this means that $\tilde{u}\left(\frac{1}{c}\right) \in X_{\delta}$. Hence $X_{\delta}$ is stable under $\mathcal{T}_{1 / c}$.

To establish Claim 3.5. it remains to prove that $\mathcal{T}_{1 / c}$ has a fixed point in $X_{\delta}$; this follows easily from the previous remarks:

$$
\begin{aligned}
\|(\tilde{V}-\tilde{u})(t)\|_{Y_{\delta}} \leq\left\|T(t, 0)\left(\tilde{V}(0)-\tilde{u}_{0}\right)\right\|_{Y_{\delta}}+\left\|\int_{0}^{t} T(t, s) \tilde{g}(s, \cdot, \cdot) d s\right\|_{Y_{\delta}} \\
\quad \leq C_{\delta} e^{-\omega_{\delta} t}\left\|\tilde{V}(0)-\tilde{u}_{0}\right\|_{Y_{\delta}}+C_{\delta}^{\prime} .
\end{aligned}
$$

Choose $p \in \mathbb{N}$ large enough such that $C_{\delta} e^{-\omega_{\delta} p / c}<1$, denote $\nu:=1-C_{\delta} e^{-\omega_{\delta} p / c}$, and let $R_{0}:=\frac{C_{\delta}^{\prime}}{\nu}$. Then

$$
\left\|(\tilde{V}-\tilde{u})\left(\frac{p}{c}\right)\right\|_{Y_{\delta}} \leq(1-\nu)\left\|\tilde{V}(0)-\tilde{u}_{0}\right\|_{Y_{\delta}}+R_{0} \nu
$$

which implies that the ball of $X_{\delta}$ centered in $\tilde{V}(0)$ and of radius $R_{0}$ :

$$
B\left(\tilde{V}(0), R_{0}\right)=\left\{\tilde{u}_{0} \in X_{\delta},\left\|\tilde{u}_{0}-\tilde{V}(0)\right\| \leq R_{0}\right\}
$$

is stable under $\left(\mathcal{T}_{1 / c}\right)^{p}$. Moreover, $\left(\mathcal{T}_{1 / c}\right)^{p}$ is a contraction on this ball. Indeed, consider $\left.\tilde{u}_{0}, \tilde{w}_{0} \in B\left(\tilde{V}(0), R_{0}\right)\right)$; then consider the solutions $\tilde{u}$ and $\tilde{w}$ of the corresponding Cauchy problems (3.15). Similarly to Section 2, the difference $\tilde{v}:=|\tilde{u}-\tilde{w}|$ satisfies

$$
\tilde{v}_{t}-\operatorname{div}(A(\xi-c t, y) D \tilde{v})+\left(B(\xi-c t, y)+c e_{1}\right) \cdot D \tilde{v}-f_{u}(\xi-c t, y, a(t, \xi, y)) \tilde{v} \geq 0,
$$

and the maximum principle implies that

$$
|\tilde{u}-\tilde{w}| \leq \tilde{v} .
$$

Now, thanks to Lemma 3.4, we obtain that

$$
\|(\tilde{u}-\tilde{w})(t)\|_{Y_{\delta}} \leq C_{\delta} e^{-\omega_{\delta} t}\left\|\tilde{u}_{0}-\tilde{w}_{0}\right\|_{Y_{\delta}}
$$

since $C_{\delta} e^{-\omega_{\delta} p / c}<1,\left(\mathcal{T}_{1 / c}\right)^{p}$ is a contraction on the ball $B\left(\tilde{V}(0), R_{0}\right)$, which implies that $\left(\mathcal{T}_{1 / c}\right)^{p}$ and then $\mathcal{T}_{1 / c}$ has a unique fixed point in $B\left(\tilde{V}(0), R_{0}\right)$. This establishes Claim 3.5 .

Step 2 (The fixed point has the right limits at $+\infty$ ). Now we prove that

$$
\lim _{\xi \rightarrow+\infty} \tilde{\phi}_{c}(t, \xi, y)=1 \text {. }
$$


We first want to prove:

Claim 3.8. $\liminf _{\xi \rightarrow+\infty, y \in \mathbb{T}^{N-1}} \tilde{\phi}_{c}(0, \xi, y)>0$.

Indeed, assume by contradiction that there exists a sequence $\left(\xi_{n}, y_{n}\right)_{n}, \xi_{n} \rightarrow$ $+\infty$, and $y_{n} \in \mathbb{T}^{N-1}$ such that $\tilde{\phi}_{c}\left(0, \xi_{n}, y_{n}\right) \rightarrow 0$ as $n \rightarrow \infty$. Let $\varepsilon>0$. Then for some $N$, we have $\tilde{\phi}_{c}\left(0, \xi_{n}, y_{n}\right) \leq \varepsilon$ for all $n \geq N$. By Harnack's inequality and parabolic estimates, there exists $C>0$ such that for all $(t, y) \in[0,1 / c] \times \mathbb{T}^{N-1}$, $\tilde{\phi}_{c}\left(t, \xi_{n}, y\right) \leq C \varepsilon$ and $\left\|D \tilde{\phi}_{c}\left(t, \xi_{n}, y\right)\right\| \leq C \varepsilon$. Take $\zeta<0$. Then integrating the equation on $[0,1 / c] \times\left[\zeta, \xi_{n}\right] \times \mathbb{T}^{N-1}$, and using the periodicity in $t$, we obtain

$$
\begin{gathered}
-\int_{0}^{1 / c} \int_{\mathbb{T}^{N-1}}\left[A(\xi-c t, y) D \tilde{\phi}_{c}(t, \xi, y) \cdot e_{1}\right]_{\zeta}^{\xi_{n}}+\int_{0}^{1 / c} \int_{\mathbb{T}^{N-1}}\left[B(\xi-c t, y) \cdot e_{1} \tilde{\phi}_{c}\right]_{\zeta}^{\xi_{n}} \\
-\int_{0}^{1 / c} \int_{\zeta}^{\xi_{n}} \int_{\mathbb{T}^{N-1}} \operatorname{div} B(\xi-c t, y) \tilde{\phi}_{c}+c \int_{0}^{1 / c} \int_{\mathbb{T}^{N-1}}\left[\tilde{\phi}_{c}(t, \xi, y)\right]_{\zeta}^{\xi_{n}} \\
=\int_{0}^{1 / c} \int_{\zeta}^{\xi_{n}} \int_{\mathbb{T}^{N-1}} f\left(\xi-c t, y, \tilde{\phi}_{c}(t, \xi, y)\right) .
\end{gathered}
$$

Since $\operatorname{div} B=0$, we have

$$
\begin{aligned}
& -\int_{0}^{1 / c} \int_{\mathbb{T}^{N-1}}\left[A(\xi-c t, y) D \tilde{\phi}_{c}(t, \xi, y) \cdot e_{1}\right]_{\zeta}^{\xi_{n}}+\int_{0}^{1 / c} \int_{\mathbb{T}^{N-1}}\left[B(\xi-c t, y) \cdot e_{1} \tilde{\phi}_{c}(t, \xi, y)\right]_{\zeta}^{\xi_{n}} \\
& +c \int_{0}^{1 / c} \int_{\mathbb{T}^{N-1}}\left[\tilde{\phi}_{c}(t, \xi, y)\right]_{\zeta}^{\xi_{n}}=\int_{0}^{1 / c} \int_{\zeta}^{\xi_{n}} \int_{\mathbb{T}^{N-1}} f\left(\xi-c t, y, \tilde{\phi}_{c}(t, \xi, y)\right) .
\end{aligned}
$$

Denote by $L\left(\zeta, \xi_{n}\right)$ the left-hand side of (3.17) and by $R\left(\zeta, \xi_{n}\right)$ the right-hand side of (3.17): (3.17) says that $L\left(\zeta, \xi_{n}\right)=R\left(\zeta, \xi_{n}\right)$, but we are going to see that this is not possible, taking the limits $\zeta \rightarrow-\infty$ and $n \rightarrow+\infty$. Indeed, first consider $R\left(\zeta, \xi_{n}\right)$ : clearly

$$
\lim _{\zeta \rightarrow-\infty, \xi_{n} \rightarrow+\infty} R\left(\zeta, \xi_{n}\right)=\int_{0}^{1 / c} \int_{-\infty}^{+\infty} \int_{\mathbb{T}^{N-1}} f\left(\xi-c t, y, \tilde{\phi}_{c}(t, \xi, y)\right)=: R_{\infty},
$$

and since $\tilde{\phi}_{c}$ is positive at least for $\xi$ close to $-\infty$ we get that $R_{\infty}>0$; hence, for $-\zeta$ and $n$ large enough,

$$
R\left(\zeta, \xi_{n}\right) \geq \frac{R_{\infty}}{2}>0
$$

Next consider $L\left(\zeta, \xi_{n}\right)$ : since $\tilde{\phi}_{c}(t, \zeta, y) \rightarrow 0$ as $\zeta \rightarrow-\infty$, the usual parabolic estimates imply that $D \tilde{\phi}_{c}(t, \zeta, y) \rightarrow 0$ as $\zeta \rightarrow-\infty$, uniformly in $(t, y) \in[0,1 / c] \times$ $\mathbb{T}^{N-1}$, and that $\tilde{\phi}_{c}(t, \zeta, y)$ and $\left\|D \tilde{\phi}_{c}(t, \zeta, y)\right\|$ are bounded by $\varepsilon$ for $-\zeta$ large enough; this remark and the choice of $\xi_{n}$ (and the consequences of the Harnack inequality that we already mentioned) imply that if $-\zeta$ and $n$ are large enough, then

$$
L\left(\zeta, \xi_{n}\right) \leq C^{\prime} \varepsilon .
$$

But now, comparing this with the fact that $L\left(\zeta, \xi_{n}\right)=R\left(\zeta, \xi_{n}\right)$ and that $R\left(\zeta, \xi_{n}\right)$ is uniformly bounded from below by a positive constant, we obtain a contradiction. Hence Claim 3.8 is proved.

Now we are ready to prove that $\lim _{\xi \rightarrow+\infty} \tilde{\phi}_{c}(t, \xi, y)=1$ : Claim 3.8 implies that $\tilde{\phi}_{c}(0)$ is bounded from below by a positive constant on some $\left[X^{\prime},+\infty\right) \times \mathbb{T}^{N-1}$; 
since $\tilde{\phi}_{c}$ is positive, it is also bounded from below by a positive constant on $\left[0, \frac{1}{c}\right] \times$ $\left\{X^{\prime}\right\} \times \mathbb{T}^{N-1}$, and then the weak maximum principle implies that $\tilde{\phi}_{c}$ is bounded from below by a positive constant $\gamma$ on $\left[0, \frac{1}{c}\right] \times\left[X^{\prime},+\infty\right) \times \mathbb{T}^{N-1}$. Now consider $\tilde{\Phi}:=1-\tilde{\phi}_{c}$ and $f(x, y, u)=(1-u) h(x, y, u)$. Since $\frac{\partial f}{\partial u}(x, y, 1)<0$, there is some $h_{0}>0$ such that $h(x, y, u) \geq h_{0}>0$ for all $(x, y, u) \in \mathbb{R} \times \mathbb{T}^{N-1} \times[\gamma, 1]$. Moreover, the function $\tilde{\Phi}$ satisfies the equation

$$
\tilde{\Phi}_{t}-\operatorname{div}(A(\xi-c t, y) D \tilde{\Phi})+\left(B(\xi-c t, y)+c e_{1}\right) \cdot D \tilde{\Phi}+h\left(\xi-c t, y, \tilde{\phi}_{c}\right) \tilde{\Phi}=0 .
$$

In the domain $\mathbb{R}_{+} \times\left[X^{\prime},+\infty\right) \times \mathbb{T}^{N-1}$, it is easy to check that the function

$$
\Psi(t, \xi, y):=\|\tilde{\Phi}(0)\|_{\infty} e^{-h_{0} t}+C e^{-\mu \xi}
$$

will be an upper solution if $\mu>0$ is small enough. Then, if $C \geq e^{\mu X^{\prime}}$, then the weak maximum principle implies that $\tilde{\Phi}(t, \xi, y) \leq \Psi(t, \xi, y)$ for all $t \geq 0, \xi \geq X^{\prime}$ and $y \in \mathbb{T}^{N-1}$. Since $\tilde{\Phi}$ is $1 / c$-periodic in time, we can evaluate the previous inequality for $t=t+N / c$ and pass to the limit as $N \rightarrow+\infty$. Thus we get $\tilde{\Phi}(t, \xi, y) \leq C e^{-\mu \xi}$. Hence, $\tilde{\Phi}(t, \xi, y) \rightarrow 0$ and $\tilde{\phi}_{c}(t, \xi, y) \rightarrow 1$ as $\xi \rightarrow+\infty$. This completes the proof of Step 2.

Step 3 (The constructed pulsating wave is increasing in time). To complete the proof of Proposition 3.3. it remains to prove that the constructed pulsating wave $\phi_{c}$ satisfies: $\partial_{t} \phi_{c}>0$, or equivalently that $\partial_{t} \tilde{\phi}_{c}+c \partial_{\xi} \tilde{\phi}_{c}>0$.

We are going to prove that

$$
\forall t^{\prime}>0, \forall t \in \mathbb{R}, \forall x \in \mathbb{R}, \forall y \in \mathbb{T}^{N-1}, \quad \phi_{c}(t, x, y) \leq \phi_{c}\left(t+t^{\prime}, x, y\right) .
$$

In the moving frame, this becomes

$$
\forall t^{\prime}>0, \forall t \in \mathbb{R}, \forall x \in \mathbb{R}, \forall y \in \mathbb{T}^{N-1}, \quad \tilde{\phi}_{c}(t, x+c t, y) \leq \tilde{\phi}_{c}\left(t+t^{\prime}, x+c t+c t^{\prime}, y\right) ;
$$

hence we want to prove that

$$
\forall t^{\prime}>0, \forall t \in \mathbb{R}, \forall \xi \in \mathbb{R}, \forall y \in \mathbb{T}^{N-1}, \quad \tilde{\phi}_{c}(t, \xi, y) \leq \tilde{\phi}_{c}\left(t+t^{\prime}, \xi+c t^{\prime}, y\right) .
$$

Fix $t^{\prime}>0$ and denote

$$
\tilde{d}(t, \xi, y)=\tilde{\phi}_{c}\left(t+t^{\prime}, \xi+c t^{\prime}, y\right)-\tilde{\phi}_{c}(t, \xi, y) .
$$

As is by now usual, there exists some function $b\left(t, \xi, y, t^{\prime}\right)$ such that

$$
\tilde{d}_{t}-\operatorname{div}(A(\xi-c t, y) D \tilde{d})+\left(B(\xi-c t, y)+c e_{1}\right) \cdot D \tilde{d}=f_{u}\left(\xi-c t, y, b\left(t, \xi, y, t^{\prime}\right)\right) \tilde{d} .
$$

Now note that $\tilde{d}$ is bounded, and moreover

$$
\tilde{d}(t, \xi, y) \sim_{\xi \rightarrow-\infty}\left(e^{r_{-}(c) c t^{\prime}}-1\right) e^{r_{-}(c) \xi} \psi_{r_{c}}(\xi-c t, y)>0 ;
$$

hence there exists $C \geq 0$ such that

$\forall \xi \in \mathbb{R}, \forall y \in \mathbb{T}^{N-1}, \quad \tilde{d}(0, \xi, y)+C \tilde{V}^{\delta}(0, \xi, y)=\tilde{d}(0, \xi, y)+C \tilde{V}^{\left(\lambda_{\delta}, \omega_{\delta}\right)}(0, \xi, y) \geq 0$.

Finally, we note that

$$
\begin{aligned}
\left(\tilde{d}+C \tilde{V}^{\delta}\right)_{t}-\operatorname{div}( & \left.A(\xi-c t, y) D\left(\tilde{d}+C \tilde{V}^{\delta}\right)\right) \\
+ & \left(B(\xi-c t, y)+c e_{1}\right) \cdot D\left(\tilde{d}+C \tilde{V}^{\delta}\right) \\
- & f_{u}\left(\xi-c t, y, b\left(t, \xi, y, t^{\prime}\right)\right)\left(\tilde{d}+C \tilde{V}^{\delta}\right) \\
& =\left(f_{u}(\xi-c t, y, 0)-f_{u}\left(\xi-c t, y, b\left(t, \xi, y, t^{\prime}\right)\right)\right) C \tilde{V}^{\delta} \geq 0 .
\end{aligned}
$$


We deduce from the weak maximum principle that, for all $(t, \xi, y) \in \mathbb{R} \times \mathbb{R} \times \mathbb{T}^{N-1}$,

$$
\left(\tilde{d}+C \tilde{V}^{\delta}\right)(t, \xi, y) \geq 0 .
$$

Now we use the fact that $\tilde{d}$ is $1 / c$-periodic in time: we fix $\tau \in[0,1 / c]$, and we evaluate this inequality at $t=\tau+N / c$; then letting $N \rightarrow+\infty$, we obtain that $\tilde{d} \geq 0$. This implies (3.19), hence (3.18), which obviously implies that $\partial_{t} \phi_{c} \geq 0$. To obtain the stronger property that we want: $\partial_{t} \phi_{c}>0$, it is sufficient to note that $\partial_{t} \phi_{c}$ is a nonnegative solution of the parabolic equation

$$
v_{t}-\operatorname{div}(A(x, y) D v)+B(x, y) \cdot D v=f_{u}\left(x, y, \phi_{c}\right) v ;
$$

hence the strong maximum principle implies that either $\partial_{t} \phi_{c}>0$ or $\partial_{t} \phi_{c}$ is identically zero. But in this last case, $\phi_{c}$ does not depend on $t$; hence

$$
\phi(t, x, y)=\phi_{t}\left(t+\frac{1}{c}, x, y\right)=\phi_{c}(t, x+1, y) .
$$

Repeating this argument, we obtain that $\phi(t, x, y)=1$, thanks to the limit as the space variable $x \rightarrow+\infty$. But this violates the other limit condition $\phi_{c}(t,-\infty, y)=$ 0 . Hence the constructed pulsating wave satisfies $\partial_{t} \phi_{c}>0$, and this concludes the proof of Step 3 and of Proposition 3.3 .

3.2. Attractive dynamics. Now we are interested in the Cauchy problem associated to (1.1). We recall that we assume that $c>c_{*}, \phi_{c}$ is a pulsating wave propagating at speed $c$, and the initial condition $u_{0}$ is trapped between two translates of $\phi_{c}$, as in Theorem 1.3. We denote

$$
N L[u]=u_{t}-\operatorname{div}(A D u)+B \cdot D u-f(x, y, u) .
$$

Hence $u$ is a solution of (1.1) if and only if $N L[u]=0$. The proof of Theorem 1.3 follows the same strategy as that of Theorem [1.1.

3.2.1. Exact and approximate shift. Given a sufficiently smooth function $m:(0,+\infty)$ $\times \mathbb{R} \times \mathbb{T}^{N-1} \rightarrow \mathbb{R}$, consider

$$
T^{(m)} \phi_{c}(t, x, y):=\phi_{c}(t+m(t, x, y), x, y) .
$$

Of course, when $m$ is identically zero, we have $T^{(0)} \phi_{c}=\phi_{c}$ and $N L\left[T^{(0)} \phi_{c}\right]=0$. Let us compute in the general case $N L\left[T^{(m)} \phi_{c}\right]$ : we have

$$
N L\left[T^{(m)} \phi_{c}\right]=\left(m_{t}-\operatorname{div}(A D m)+\left(B-2 A \frac{D \partial_{t} \phi_{c}}{\partial_{t} \phi_{c}}\right) \cdot D m-\frac{\partial_{t t} \phi_{c}}{\partial_{t} \phi_{c}} A D m . D m\right) \partial_{t} \phi_{c},
$$

where, on the right-hand side, $m$ and its derivatives are evaluated at $(t, x, y)$, the coefficients $A, B$ are evaluated at $(x, y)$, and $\partial_{t} \phi_{c}$ and its derivatives are evaluated at $(t+m(t, x, y), x, y)$. Hence $T^{(m)} \phi_{c}$ is a solution of (1.1) if and only if

$$
m_{t}-\operatorname{div}(A D m)+\left(B-2 A \frac{D \partial_{t} \phi_{c}}{\partial_{t} \phi_{c}}\right) \cdot D m-\frac{\partial_{t t} \phi_{c}}{\partial_{t} \phi_{c}} A D m \cdot D m=0 .
$$

Since, given $(x, y)$, the function $t \mapsto \phi_{c}(t, x, y)$ is strictly increasing, the exact shift

$$
m^{*}(t, x, y):=\left(\phi_{c}(\cdot, x, y)\right)^{-1}(u(t, x, y))-t
$$

satisfies $u(t, x, y)=\phi_{c}\left(t+m^{*}(t, x, y), x, y\right)$ and (3.21). 
Now, we replace the exact shift by an approximate one. To do it, recall that we proved in Proposition 3.3 the expansion

$$
\begin{aligned}
\phi_{c}(t, x, y) & =e^{r_{-}(c)(x+c t)} \psi_{r_{c}}(x, y)+O\left(e^{\left(r_{-}(c)+\delta\right)(x+c t)}\right) \\
& =V(t, x, y)+O\left(e^{\left(r_{-}(c)+\delta\right)(x+c t)}\right) .
\end{aligned}
$$

Now, due to elliptic regularity, the exponential deviation of $\phi_{c}$ from $V$ also holds for the derivatives: indeed, $\phi_{c}$ and $V$ satisfy

$$
\left\{\begin{array}{l}
\left(\phi_{c}\right)_{t}-\operatorname{div}\left(A(x, y) D \phi_{c}\right)+B(x, y) \cdot D \phi_{c}=f\left(x, y, \phi_{c}\right) \\
V_{t}-\operatorname{div}(A(x, y) D V)+B(x, y) \cdot D V=f_{u}(x, y, 0) V
\end{array}\right.
$$

hence the difference $\phi_{c}-V$ satisfies (in the variable $(t, \xi, y)$ )

$$
\begin{aligned}
&\left(\tilde{\phi}_{c}-\tilde{V}\right)_{t}-\operatorname{div}\left(A(\xi-c t, y) D\left(\tilde{\phi}_{c}-\tilde{V}\right)\right)+\left(B(\xi-c t, y)+c e_{1}\right) \cdot D\left(\tilde{\phi}_{c}-\tilde{V}\right) \\
&-f_{u}(\xi-c t, y, 0)\left(\tilde{\phi}_{c}-\tilde{V}\right)=f\left(\xi-c t, y, \tilde{\phi}_{c}\right)-f_{u}(\xi-c t, y, 0) \tilde{\phi}_{c} .
\end{aligned}
$$

Since

$$
\tilde{\phi}_{c}-\tilde{V}=O\left(e^{\left(r_{-}(c)+\delta\right) \xi}\right), \quad f\left(\xi-c t, y, \tilde{\phi}_{c}\right)-f(\xi-c t, y, 0) \tilde{\phi}_{c}=O\left(e^{\left(r_{-}(c)+\delta\right) \xi}\right),
$$

we deduce that the derivatives of $\tilde{\phi}_{c}-\tilde{V}$ satisfy the same estimates.

Hence, neglecting in a first approach the residual term, we obtain

$$
\begin{gathered}
\partial_{t} \phi_{c}(t, x, y)=c r_{-}(c) e^{r_{-}(c)(x+c t)} \psi_{r_{c}}(x, y)+\ldots, \\
\partial_{t t} \phi_{c}(t, x, y)=c^{2} r_{-}(c)^{2} e^{r_{-}(c)(x+c t)} \psi_{r_{c}}(x, y)+\ldots \\
D \partial_{t} \phi_{c}(t, x, y)=c r_{-}(c)^{2} e^{r_{-}(c)(x+c t)} \psi_{r_{c}}(x, y) e_{1}+c r_{-}(c) e^{r_{-}(c)(x+c t)} D \psi_{r_{c}}(x, y)+\ldots
\end{gathered}
$$

This invites us to choose the approximate shift as the solution of $(3.22)$

$$
\left\{\begin{array}{l}
m_{t}-\operatorname{div}(A D m)+\left(B-2 A \frac{D \psi_{r_{c}}}{\psi_{r_{c}}}-2 r_{-}(c) A e_{1}\right) \cdot D m-c r_{-}(c) A D m \cdot D m=0 \\
m(0, x, y)=m_{0}(x, y)
\end{array}\right.
$$

the coefficients of this equation being the equivalents as $x+c t \rightarrow-\infty$ of the coefficients of (3.21), and where the initial condition $m_{0}$ is chosen as follows: as we did in subsection 2.3. we choose $m_{0}=m^{*}(0)$ if $m^{*}(0)$ is $C^{1}$ and bounded in norm $C^{1}$; in the general case, it can be checked, as in subsection 2.3. that $m^{*}(1)$ is $C^{1}$ and bounded in norm $C^{1}$, thanks to the definition of the exact shift $m^{*}$ and the assumption on the initial condition $u_{0}$ (trapped between two translates of $\phi_{c}$ ); then we solve the Cauchy problem related to $m$ on the time interval $(1,+\infty)$, choosing $m(1)=m^{*}(1)$ as the initial condition. In the following we assume that $m^{*}(0)$ is $C^{1}$ and bounded, and thus $m_{0}=m^{*}(0)$.

Now we are ready to sum up the properties of the approximate shift $m$ that will be of interest to us:

Proposition 3.9. The solution $m$ of (3.22) has the following properties:

(i) $m$ is bounded on $(0,+\infty) \times \mathbb{R} \times \mathbb{T}^{N-1}$, and more precisely

$$
\forall t>0, \forall x \in \mathbb{R}, \forall y \in \mathbb{T}^{N-1}, \quad m(t, x, y) \in\left[-\left\|m^{*}(0)\right\|_{\infty},\left\|m^{*}(0)\right\|_{\infty}\right] ;
$$

(ii) we have $\lim _{t \rightarrow+\infty}\left\|\left(m_{t}, D m, D^{2} m\right)(t, .)\right\|_{L^{\infty}\left(\mathbb{R} \times \mathbb{T}^{N-1}\right)}=0$. 
Before proving Proposition 3.9, let us introduce the shifted wave associated to the approximate shift $m$ solution of (3.22):

$$
u^{a p p}(t, x, y):=T^{(m)} \phi_{c}(t, x, y)=\phi_{c}(t+m(t, x, y), x, y) .
$$

Of course $u^{a p p}(0, x, y)=u_{0}(x, y)$, but $u^{a p p}$ is not a solution of (1.1), since $m$ is not a solution of (3.21). However, awe can check that $N L\left[u^{a p p}\right]$ decays faster than $e^{r_{-}(c)(x+c t)}$ as $x+c t \rightarrow-\infty$, a property that was essential in the proof of Theorem 1.1. indeed, we already know that

$$
\frac{N L\left[u^{a p p}\right]}{\partial_{t} \phi_{c}}=m_{t}-\operatorname{div}(A D m)+\left(B-2 A \frac{D \partial_{t} \phi_{c}}{\partial_{t} \phi_{c}}\right) \cdot D m-\frac{\partial_{t t} \phi_{c}}{\partial_{t} \phi_{c}} A D m \cdot D m,
$$

where $m$ and its derivatives are evaluated at $(t, x, y)$, the coefficients $A, B$ are evaluated at $(x, y)$, and $\partial_{t} \phi_{c}$ and its derivatives are evaluated at $(t+m(t, x, y), x, y)$; now taking care of the residual term $\phi_{c}-V$, it is easy to check that

$$
\begin{gathered}
\frac{D \partial_{t} \phi_{c}(t+m(t, x, y), x, y)}{\partial_{t} \phi_{c}(t+m(t, x, y), x, y)}=\frac{D \psi_{r_{c}}(x, y)}{\psi_{r_{c}}(x, y)}+r_{-}(c) e_{1}+O\left(e^{\delta(x+c t)}\right), \\
\frac{\partial_{t t} \phi_{c}}{\partial_{t} \phi_{c}}=c r_{-}(c)+O\left(e^{\delta(x+c t)}\right)
\end{gathered}
$$

which yields that

$$
\frac{N L\left[u^{a p p}\right]}{\partial_{t} \phi_{c}}=O\left(e^{\delta(x+c t)}\right)(|D m|+A D m . D m)
$$

hence

$$
N L\left[u^{a p p}\right]=O\left(e^{\delta(x+c t)}\right)\left(|D m|+|D m|^{2}\right) \partial_{t} \phi_{c}=O\left(e^{\left(r_{-}(c)+\delta\right)(x+c t)}\right)\left(|D m|+|D m|^{2}\right) .
$$

This estimate and the results of Proposition 3.9 will be the main arguments to prove the convergence result of Theorem 1.3 (the convergence of the shifted wave $u^{a p p}$ to $u$ ), as explained in the following subsection. To conclude this subsection, we prove the properties of $m$ :

Proof of Proposition 3.9. By the Hopf-Cole transform $s(t, x, y)=e^{c r_{-}(c) m(t, x, y)}$, we have

$$
s_{t}-\operatorname{div}(A D s)+\left(B-2 A \frac{D \psi_{r_{c}}}{\psi_{r_{c}}}-2 r_{-}(c) A e_{1}\right) \cdot D s=0
$$

with initial datum $s(0)=e^{c r_{-}(c) m(0)}$. Then the standard theory on linear parabolic equations implies (i) (weak maximum principle), so it only remains to prove (ii). For this, we generalize a result proved by L. Rossi in the elliptic case (see [27]), and the idea is the same. The proof will result from the following three assertions:

Claim 3.10. Every element of the $\omega$ limit set of the initial condition $s(0)$ generates a bounded and (time) global solution of (3.26).

Claim 3.11. Every bounded and global solution of (3.26) is constant in space and time.

Claim 3.12. $\lim _{t \rightarrow+\infty}\left\|\left(s_{t}, D s, D^{2} s\right)(t, .)\right\|_{L^{\infty}\left(\mathbb{R} \times \mathbb{T}^{N-1}\right)}=0$. 
Of course Claim 3.12 immediately implies (iii) of Proposition 3.9. Hence it remains to prove these three claims.

Claim 3.10 is classical: $\{s(t), t \geq 0\}$ is relatively compact in $C^{0}\left(\mathbb{R} \times \mathbb{T}^{N-1}\right)$. Choose $t_{n} \rightarrow+\infty$ such that $s\left(t_{n}\right)$ converges locally uniformly to $s_{\infty}$, and consider the solution $S$ of the problem

$$
\left\{\begin{array}{l}
S_{t}-\operatorname{div}(A D S)+\left(B-2 A \frac{D \psi_{r_{c}}}{\psi_{r_{c}}}-2 r_{-}(c) A e_{1}\right) \cdot D S=0, \\
S(0)=s_{\infty} .
\end{array}\right.
$$

Of course $S$ is well defined on $[0,+\infty)$, but we are going to prove that $S$ is also well defined on $[-1,1]$ and satisfies the problem on $(-1,1)$ :

- first, by continuity, we have that $s\left(t_{n}+t\right) \rightarrow S(t)$ for all $t \geq 0$;

- next, choose $n_{1}$ such that $t_{n} \geq 2$ for all $n \geq n_{1}$, and consider the sequence $\left(s_{n}\right)_{n}, s_{n}(t, x, y)=s\left(t+t_{n}, x, y\right)$ : it is relatively compact in $C^{0}\left([-1,1] \times \mathbb{R} \times \mathbb{T}^{N-1}\right)$; hence it has a subsequence $\left(s_{\varphi(n)}\right)_{n}$ that converges locally uniformly to some $S_{1}$. Then $S_{1}(t)=S(t)$ for all $t \in[0,1]$, and we derive from parabolic estimates that $S_{1}$ is a solution of the problem (3.27) on the time interval $(-1,1)$.

Hence this allows us to extend $S$ on the time interval $[-1,0]$, and then the same reasoning implies that it is a global solution, which proves Claim 3.10.

To prove Claim 3.11, we consider a global solution $S$ of (3.27), and we are going to prove that for all vectors $e$ of the canonical basis of $\mathbb{R}^{N}$,

$$
\lim _{|t, x, y| \rightarrow+\infty}(S(t,(x, y)+e)-S(t, x, y))=0 .
$$

Consider

$$
m:=\liminf _{|t, x, y| \rightarrow+\infty}(S(t,(x, y)+e)-S(t, x, y)) .
$$

There exists $\left(t_{n}, x_{n}, y_{n}\right)_{n}$ such that

$$
\lim _{n \rightarrow+\infty}\left(S\left(t_{n},\left(x_{n}, y_{n}\right)+e\right)-S\left(t_{n}, x_{n}, y_{n}\right)\right)=m .
$$

Up to the extraction of a subsequence we may assume that

$$
\lim _{n \rightarrow+\infty} S\left(t+t_{n},(x, y)+\left(x_{n}, y_{n}\right)+e\right) \text { exists in } C_{\text {loc }}^{2}\left(\mathbb{R} \times \mathbb{R} \times \mathbb{T}^{N-1}\right) .
$$

Call it $S_{\infty}(t, x, y)$. Because we are dealing with a lim inf in (3.29), we have

$$
S_{\infty}(t,(x, y)+e)-S_{\infty}(t, x, y) \geq m ;
$$

hence, because it is equal to $m$ at $(0,0,0)$, it is equal to $m$ everywhere by the strong maximum principle (this is the only place where we use the equation for $S$ ). Therefore, for all $k$ :

$$
S_{\infty}(t,(x, y)+k e)-S_{\infty}(t, x, y)=k m,
$$

a contradiction with the boundedness of $S$ if $m \neq 0$; thus $m=0$. In the same way,

$$
\limsup _{|t, x, y| \rightarrow+\infty}(S(t,(x, y)+e)-S(t, x, y))=0 ;
$$

hence (3.28) is proved. This implies that $S(t,(x, y)+e)-S(t, x, y)$ has a maximum and a minimum. Thus, using twice the strong maximum principle, it is periodic in $(x, y)$, hence constant. Claim 3.11 is proved.

Claim 3.12 is now an easy consequence of Claims 3.10 and 3.11, using parabolic estimates. Take the solution $s$ of $(3.26)$. The only possible limit for its space derivatives is 0 : indeed, assume $D s\left(t_{n}, x_{n}, y_{n}\right) \rightarrow \ell$, and extract a subsequence of 
$\left(s\left(t_{n}\right)_{n}\right.$ that converges locally uniformly. Hence its limit is a global solution, thus a constant, and parabolic estimates imply that $D s\left(t_{n}\right) \rightarrow 0$; hence $\ell=0$. Then $\lim _{t \rightarrow+\infty}\|D s(t, .)\|_{L^{\infty}\left(\mathbb{R} \times \mathbb{T}^{N-1}\right)}=0$, and in the same way $\lim _{t \rightarrow+\infty}\left\|D^{2} s(t, .)\right\|_{L^{\infty}\left(\mathbb{R} \times \mathbb{T}^{N-1}\right)}$ $=0$; finally the equation shows that $\lim _{t \rightarrow+\infty}\left\|s_{t}(t, .)\right\|_{L^{\infty}\left(\mathbb{R} \times \mathbb{T}^{N-1}\right)}=0$, which concludes the proof of Claim 3.12, and of Proposition 3.9.

3.2.2. The difference between the solution and the shifted wave: Proof of Theorem 1.3. Now we have everything we need to study the difference $u-u^{a p p}$ and prove that it converges to 0 uniformly in space. The arguments are very close to the ones used in the proof of Theorem 1.1, subsection 2.5. We denote

$$
v(t, x, y):=\left(u-u^{a p p}\right)(t, x, y), \quad g(t, x, y):=N L\left[u^{a p p}\right](t, x, y),
$$

and by $\tilde{w}$ the solution of the Cauchy problem

$$
\left\{\begin{array}{l}
\tilde{w}_{t}-\operatorname{div}(A(\xi-c t, y) D \tilde{w})+\left(B(\xi-c t, y)+c e_{1}\right) \cdot D \tilde{w}-f_{u}(\xi-c t, y, 0) \tilde{w}=|\tilde{g}| \\
\tilde{w}(0)=\tilde{v}(0)=0
\end{array}\right.
$$

We are going to prove the following facts (analogues to Claims 2.2 2.4):

Claim 3.13. For all $t>0$, all $\xi \in \mathbb{R}$ and all $y \in \mathbb{T}^{N-1}$, we have $|\tilde{v}(t, \xi, y)| \leq \widetilde{w}(t, \xi, y)$.

Claim 3.14. For all $\xi_{0} \in \mathbb{R}, \sup _{\xi \leq \xi_{0}, y \in \mathbb{T}^{N-1}} \widetilde{w}(t, \xi, y) \rightarrow 0$ as $t \rightarrow+\infty$.

Claim 3.15. There exists $\xi_{0} \in \mathbb{R}$ such that $\sup _{\xi \geq \xi_{0}, y \in \mathbb{T}^{N-1}}|\widetilde{v}(t, \xi, y)| \rightarrow 0$ as $t \rightarrow+\infty$.

It is clear that Claims 3.133 .15 imply Theorem 1.3. They imply that

$$
\|\widetilde{v}(t)\|_{L^{\infty}\left(\mathbb{R} \times \mathbb{T}^{N-1}\right)} \rightarrow 0
$$

as $t \rightarrow+\infty$; hence $\left\|u(t)-u^{a p p}(t)\right\|_{L^{\infty}\left(\mathbb{R} \times \mathbb{T}^{N-1}\right)}=\|v(t)\|_{L^{\infty}\left(\mathbb{R} \times \mathbb{T}^{N-1}\right)} \rightarrow 0$ as $t \rightarrow$ $+\infty$. Hence it remains to prove these claims.

Claim 3.13 is a consequence of the weak maximum principle and Kato's inequality, just as in Section 2. Claim 3.14 is a consequence of Lemma 3.4 by Duhamel's formula, $\tilde{w}(t)=\int_{0}^{t} T(t, s)|\tilde{g}(s)| d s$; thanks to (3.25), we have $|\tilde{g}(s)|=$ $O(1) e^{\left(r_{-}(c)+\delta\right) \xi}\|D m(s)\|_{L^{\infty}\left(\mathbb{R} \times \mathbb{T}^{N-1}\right)}$ and since $|\tilde{g}(s)| \in Y_{\delta}$, Lemma 3.4 says that

$$
\|T(t, s)\|_{Y_{\delta}}=O(1) e^{-\omega_{\delta}(t-s)} .
$$

Hence we obtain that

$$
\|\tilde{w}(t)\|_{Y_{\delta}} \leq O(1) \int_{0}^{t} e^{-\omega_{\delta}(t-s)}\|D m(s)\|_{L^{\infty}\left(\mathbb{R} \times \mathbb{T}^{N-1}\right)} d s .
$$

Finally, Proposition 3.9 and the usual properties of convolution imply that $\|\tilde{w}(t)\|_{Y_{\delta}}$ $\rightarrow 0$ as $t \rightarrow+\infty$; hence Claim 3.14 is proved.

Claim 3.15 is a consequence of Claim 3.14 since the initial condition is trapped between $\phi_{c}(-M)$ and $\phi_{c}(M)$, we know that for all $t>0, u(t)$ is trapped between $\phi_{c}(t-M)$ and $\phi_{c}(t+M)$; hence $\tilde{u}(t, \xi, y) \geq \phi_{c}(t-M, \xi-c t, y)$. Choosing $k \in \mathbb{N}$ such that $\frac{k}{c} \geq M$, we obtain that

$$
\tilde{u}(t, \xi, y) \geq \phi_{c}\left(t-\frac{k}{c}, \xi-c t, y\right)=\phi_{c}(t, \xi-c t-k, y)=\tilde{\phi}_{c}(t, \xi-k, y) .
$$


Now, there exists $q_{0}>0, \eta>0$ such that $-f_{u}(x, y, s) \geq q_{0}$ for all $x \in \mathbb{R}$, all $y \in \mathbb{T}^{N-1}$ and all $s \in(1-\eta, 1)$; since there exists $\xi_{0}$ large enough such that $\tilde{\phi}_{c}(t, \xi-k, y) \geq 1-\eta$ for all $t>0$, all $\xi \geq \xi_{0}$ and all $y \in \mathbb{T}^{N-1}$, we see that $\tilde{u}(t, \xi, y) \geq 1-\eta$ for all $t>0$, all $\xi \geq \xi_{0}$ and all $y \in \mathbb{T}^{N-1}$. The same property holds for $u^{a p p}$; hence we obtain the following, valid for all $(t, \xi, y)$ :

$$
\tilde{b}(t, \xi, y) \geq 1-\eta, \quad \text { and } \quad-f_{u}(\xi-c t, y, \tilde{b}(t, \xi, y)) \geq q_{0}>0 .
$$

Then the difference $\tilde{v}$ satisfies, for all $t>0, \xi \geq \xi_{0}, y \in \mathbb{T}^{N-1}$ :

$$
\tilde{v}_{t}-\operatorname{div}(A(\xi-c t, y) D \tilde{v})+\left(B(\xi-c t, y)+c e_{1}\right) \cdot D \tilde{v}-f_{u}(\xi-c t, y, \tilde{b}(t, \xi, y)) \tilde{v}=\tilde{g},
$$

and $\tilde{v}\left(t, \xi_{0}, y\right) \rightarrow 0$ as $t \rightarrow+\infty$, and $\tilde{v}(0, \xi, y)=0$ as $\xi \geq \xi_{0}, y \in \mathbb{T}^{N-1}$. It follows from (3.24) that $\tilde{g}(t, \xi, y)=O(1)\|D m(t)\|_{L^{\infty}\left(\mathbb{R} \times \mathbb{T}^{N-1}\right)}$; then there exists a supersolution of this problem of the form $k(t)$, with $k$ decaying sufficiently slowly to 0 as $t \rightarrow+\infty$ ( $k$ has to decay more slowly than $\left.\|D m(t)\|_{L^{\infty}\left(\mathbb{R} \times \mathbb{T}^{N-1}\right)}\right)$; hence Claim 3.15 is proved, and the proof of Theorem 1.3 is complete.

3.3. Interpretation of Theorem 1.3. We have therefore an effective equation for the local shift, but the information provided so far is rather scarce. In fact, one could very well ask about the point of developing the above computations, all the more as the Berestycki-Hamel argument (see [4]) would probably apply here with no real modifications. The extra information is provided by extracting from Norris 22 the following facts. Let us consider the linear parabolic equation, with periodic and without zero-order coefficients

$$
u_{t}-\operatorname{div}(a(X) D u)+b(X) \cdot D u=0,
$$

where $a$ and $b$ are reasonably (for instance Hölder) smooth, with values from $\mathbb{T}^{N}$ into, respectively, the set of positive definite matrices and $\mathbb{R}^{N}$. There is (KreinRutman Theorem plus some elementary functional analysis) a unique function $e^{*} \in$ $C^{2}\left(\mathbb{T}^{N}\right)$ solving

$$
-\operatorname{div}\left(a D e^{*}+e^{*} b\right)=0, \quad e^{*}>0, \int_{\mathbb{T}^{N}} e^{*}=1
$$

Indeed, denoting

$$
L_{a, b} u:=-\operatorname{div}(a(X) D u)+b(X) \cdot D u,
$$

we see that $L_{a, b}(1)=0$; hence 0 is the principal eigenvalue of $L_{a, b}$. Now denote by $L_{a, b}^{*}$ the adjoint of $L_{a, b}$ :

$$
L_{a, b}^{*} u^{*}=-\operatorname{div}\left(a D u^{*}+u^{*} b\right) ;
$$

consider $\mu^{*}$ to be the principal eigenvalue of $L_{a, b}^{*}$, and $e^{*}$ an associated positive eigenfunction. Since

$$
\mu^{*}\left\langle 1, e^{*}\right\rangle=\left\langle 1, L_{a, b}^{*} e^{*}\right\rangle=\left\langle L_{a, b} 1, e^{*}\right\rangle=0,
$$

we obtain that $\mu^{*}=0$, which implies the existence of $e^{*}$ satisfying (3.32).

Now set

$$
\bar{b}=\int_{\mathbb{T}^{N}}\left(a D e^{*}+e^{*} b\right) d X .
$$


Let $\pi(t, x, y)$ be the fundamental solution of (3.31). Here and only here, we denote by $x$ and $y$ the generic variables of $\mathbb{R}^{N}$, in order to distinguish them from the generic variable of $\mathbb{T}^{N}$. Then

Theorem $3.16([22])$. There is a constant, symmetric positive definite matrix $\bar{a}$, and $C>0$ such that

$$
e^{-C(V E+\sqrt{E}))} G_{\bar{a}}(t, x+\bar{b} t-y) \leq \pi(t, x, y) \leq C E^{N / 2} e^{C V E} G_{\bar{a}}(t, x+\bar{b} t-y)
$$

with the following notation:

- $G_{\bar{a}}$ is the image of the usual Gaussian by $\bar{a}: G_{\bar{a}}(t, x)=\frac{1}{\sqrt{\operatorname{det} \bar{a}}(4 \pi t)^{N / 2}} e^{-\frac{\bar{a} x \cdot x}{4 t}}$,

- $V$ and $E$ are corrective factors: $V=\inf \left(1, \frac{|y-x-\bar{b} t|}{t}\right)$ and $E=1+\frac{|y-x-\bar{b} t|^{2}}{t}$.

In other words, the fundamental solution of (3.31), shifted by an amount $\bar{b} t$, has a purely diffusive behavior: it is quasi-Gaussian. This theorem does not imply Proposition 3.3, but it makes it much clearer. Indeed, let us apply it and compute the effective shift $\bar{b}$ related to the parabolic equation (3.26) with periodic coefficients satisfied by the Hopf-Cole transform $s$ of the approximate shift $m$. For this, come back to our usual notation, and set, for $p(x, y) \in C^{2}\left(\mathbb{R} \times \mathbb{T}^{N-1}\right)$ :

$$
L_{c} p:=-\operatorname{div}(A D p)+\left(B-2 A \frac{D \psi_{r_{c}}}{\psi_{r_{c}}}-2 r_{-}(c) A e_{1}\right) \cdot D p
$$

Let $p_{c}^{*}$ solve

$$
L_{c}^{*} p_{c}^{*}=0, \quad p_{c}^{*}>0 .
$$

We are going to find an expression for $p_{c}^{*}$ involving $\psi_{r_{c}}$, an expression that will be useful to compute the effective shift $\bar{b}$. Consider the operator $L^{\left(\psi_{r_{c}}\right)}$ defined by

$$
L^{\left(\psi_{r_{c}}\right)} q:=\psi_{r_{c}} L_{c}\left(\frac{q}{\psi_{r_{c}}}\right)
$$

We have

$$
\begin{aligned}
\psi_{r_{c}} L_{c}\left(\frac{q}{\psi_{r_{c}}}\right)= & -\operatorname{div}(A D q)+\left(B-2 r_{-}(c) A e_{1}\right) \cdot D q \\
& -\frac{q}{\psi_{r_{c}}}\left(-\operatorname{div}\left(A D \psi_{r_{c}}\right)+\left(B-2 r_{-}(c) A e_{1}\right) \cdot D \psi_{r_{c}}\right) .
\end{aligned}
$$

Remember that $\psi_{r_{c}}$ is an eigenfunction of $L\left(r_{-}(c)\right): L\left(r_{-}(c)\right) \psi_{r_{c}}=\mu_{1}\left(r_{-}(c)\right) \psi_{r_{c}}$; the definition (3.3) of $L\left(r_{-}(c)\right)$ gives us that

$$
\begin{aligned}
& \psi_{r_{c}} L_{c}\left(\frac{q}{\psi_{r_{c}}}\right)=-\operatorname{div}(A D q)+\left(B-2 r_{-}(c) A e_{1}\right) \cdot D q \\
& \quad+q\left(-r_{-}(c)^{2} A e_{1} \cdot e_{1}-r_{-}(c) \operatorname{div}\left(A e_{1}\right)+r_{-}(c) B_{1}-f_{u}(X, 0)-\mu_{1}\left(r_{-}(c)\right)\right) .
\end{aligned}
$$

Hence we obtain that

$$
L^{\left(\psi_{r_{c}}\right)} q=\psi_{r_{c}} L_{c}\left(\frac{q}{\psi_{r_{c}}}\right)=\left(L\left(r_{-}(c)\right)-\mu_{1}\left(r_{-}(c)\right)\right) q
$$

This implies that 0 is the principal eigenvalue of $L^{\left(\psi_{r_{c}}\right)}$, and $\psi_{r_{c}}$ is an associated eigenfunction. The adjoint operator $\left(L^{\left(\psi_{r_{c}}\right)}\right) *$ is given by

$$
\left(L^{\left(\psi_{r_{c}}\right)}\right)^{*} q^{*}=\frac{1}{\psi_{r_{c}}} L_{c}^{*}\left(\psi_{r_{c}} q^{*}\right)
$$


Its principal eigenvalue is also 0; denote by $\psi_{r_{c}}^{*}$ an associated (positive) eigenfunction:

$$
\left(L^{\left(\psi_{r_{c}}\right)}\right)^{*} \psi_{r_{c}}^{*}=0, \quad \psi_{r_{c}}^{*}>0
$$

Then we obtain that

$$
L_{c}^{*}\left(\psi_{r_{c}} \psi_{r_{c}}^{*}\right)=\psi_{r_{c}}\left(L^{\left(\psi_{r_{c}}\right)}\right)^{*} \psi_{r_{c}}^{*}=0 .
$$

Since $\psi_{r_{c}} \psi_{r_{c}}^{*}$ is positive, up to a suitable normalization we have

$$
p_{c}^{*}=\psi_{r_{c}} \psi_{r_{c}}^{*}
$$

Now calling $\bar{b}_{1}$ the first component of the effective drift $\bar{b}$ given by (3.33), we have:

$$
\begin{aligned}
\bar{b}_{1} & =\int_{\mathbb{T}^{N}}\left(A D\left(\psi_{r_{c}} \psi_{r_{c}}^{*}\right) \cdot e_{1}+\psi_{r_{c}} \psi_{r_{c}}^{*}\left(B_{1}-2 r_{-}(c) A e_{1} \cdot e_{1}-2 \frac{A D \psi_{r_{c}}}{\psi_{r_{c}}} \cdot e_{1}\right)\right) d X \\
& =\int_{\mathbb{T}^{N}}\left(-2 \psi_{r_{c}}^{*} A e_{1} \cdot D \psi_{r_{c}}-\psi_{r_{c}} \psi_{r_{c}}^{*}\left(-B_{1}+2 r_{-}(c) A e_{1} \cdot e_{1}+\operatorname{div}\left(A e_{1}\right)\right)\right) d X
\end{aligned}
$$

This seemingly hopeless expression has an interpretation. Indeed, differentiating the equality

$$
L(\lambda) \psi_{\lambda}=\mu_{1}(\lambda) \psi_{\lambda}
$$

with respect to $\lambda$ (recall that we have every right to do it because $\lambda \mapsto \mu_{1}(\lambda)$ is real analytic, $\psi_{\lambda}$ is a simple eigenfunction, hence an analytic function of $\lambda$ ), multiplying by $\psi_{\lambda}^{*}$ and integrating over $\mathbb{T}^{N}$ we obtain, using (3.2) and (3.6):

$$
\bar{b}_{1}=\frac{d \mu_{1}}{d \lambda}\left(r_{-}(c)\right)=V_{*}(c)-c,
$$

and we know that $V_{*}(c)>0$.

Conclusion: In the reference frame of the pulsating wave, i.e. with horizontal drift $-c e_{1}$, the dynamics of the local shift $m(t, x, y)$ can be decomposed into

(1) an effective drift with horizontal component $V_{*}(c)$,

(2) the dynamics of a pure diffusion equation.

Moreover, exactly as in Section 2, there is a nonconvergence result.

Proposition 3.17. Assume that the initial shift $m_{0}$ is such that $S_{0}=e^{r_{-}(c) m_{0}}$ oscillates slowly at $-\infty$ :

$$
\begin{aligned}
& \forall \varepsilon>0, \exists A>0, \exists \eta>0, \forall z, z^{\prime} \leq-A \\
& \quad \text { and } 1-\eta \leq \frac{z}{z^{\prime}} \leq 1+\eta \Longrightarrow\left|S_{0}(z, y)-S_{0}\left(z^{\prime}, y\right)\right| \leq \varepsilon .
\end{aligned}
$$

Then the Hopf-Cole transform of the approximate shift $m$ satisfies

$$
s(t, 0)=S_{0}\left(-V_{*}(c) t, y\right)+o(1)
$$

The proof consists in rewriting that of Proposition 2.7 the Gaussian heat kernel being replaced by the lower or upper estimates provided by Theorem 3.16 


\section{The General MOdel 1.1) IN ONE SPACE DIMENSION: \\ Proof of TheOrem 1.4}

We are not going to recall the preliminary steps: the whole point is to compute, as explicitly as possible, the solution of (3.22), which is now:

$$
m_{t}-m_{x x}-2\left(\frac{\psi_{c}^{\prime}}{\psi_{c}}+r_{c}\right) m_{x}-c r_{c} m_{x}^{2}, \quad \phi_{c}\left(m_{0}(x), x\right)=u_{0}(x),
$$

where we have denoted, for short: $r_{c}:=r_{-}(c)$ and $\psi_{r_{c}}=\psi\left(. ; r_{-}(c)\right)$. We consider the Hopf-Cole transforms of (4.1):

$$
s(t, x)=e^{c r_{c} m(t, x)}, \quad q(t, x)=\psi_{r_{c}}(x) s(t, x)=\psi_{r_{c}}(x) e^{c r_{c} m(t, x)} .
$$

With the notation $a(x)=c r_{c}-r_{c}{ }^{2}-f_{u}(x, 0)$, and since $-\psi_{r_{c}}^{\prime \prime}-2 r_{c} \psi_{r_{c}}^{\prime}+a(x) \psi_{r_{c}}=0$, we get the following linear equation for $q$ :

$$
\left\{\begin{array}{l}
q_{t}+L s:=q_{t}-q_{x x}-2 r_{c} q_{x}+a(x) q=0 \\
q(0, x)=q_{0}(x):=\psi_{r_{c}}(x) e^{c r_{c} m_{0}(x)}
\end{array}\right.
$$

Note that $q_{0}$ is bounded. So, instructed by the preceding sections, we will compute $e^{-t L} q_{0}$, with $q_{0}$ bounded and uniformly continuous.

Let us start by recalling that $L$ is a sectorial operator in $B U C(\mathbb{R})[29]$. Thus $-L$ generates an analytic semigroup [16], and

$$
q(t, x)=e^{-t L} q_{0}(x)=\frac{1}{2 i \pi} \int_{\gamma} e^{t \lambda}(\lambda I+L)^{-1} q_{0}(x) d \lambda,
$$

where $\gamma$ is a wedge-like path bypassing a sector $\Sigma$ containing the spectrum of $-L$ (see Figure 1).

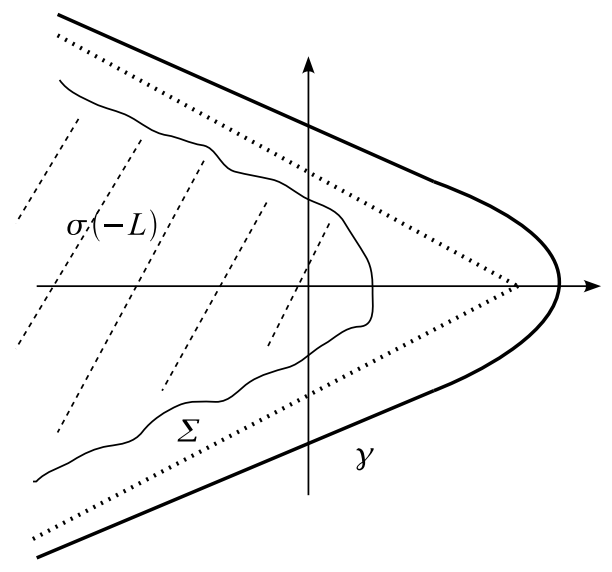

FiguRE 1. Integral path $\gamma$

Our main goal is to prove that $\left\|m_{x}(t, \cdot)\right\|_{\infty}=O\left(t^{-1 / 2}\right)$. To obtain such an estimate using the expression for $q$ obtained in (4.3), first we are going to study the spectrum of $L$ : we will prove (this is not completely obvious) that if $\lambda \in \sigma(L)$, then $\lambda=0$ or $\Re e(\lambda)>0$. This will allow us to deform the path $\gamma$ into the axis $i \mathbb{R}$, and then Fourier analysis will lead us to a Gaussian integral. Then we estimate the Gaussian integral. This last part is (although quite instructive about the heat kernel structure of $e^{-t L}$ ) tedious and can be skipped at first glance. 
4.1. Preliminaries: Basic Floquet theory. If $\lambda I+L$ is invertible, then $v(x, \lambda):=$ $(\lambda I+L)^{-1} q_{0}(x)$ is a bounded function of $x$ that satisfies the linear differential equation of the second order

$$
-v^{\prime \prime}-2 r_{c} v^{\prime}+a(x) v+\lambda u=q_{0} .
$$

We are so led to study the bounded solutions of (4.4). Equation (4.4) can be written under the form

$$
V^{\prime}=A_{\lambda}(x) V+G(x)=\left(\begin{array}{cc}
0 & 1 \\
a(x)+\lambda & -2 r_{c}
\end{array}\right)\left(\begin{array}{c}
v(x) \\
v^{\prime}(x)
\end{array}\right)+\left(\begin{array}{c}
0 \\
-q_{0}(x)
\end{array}\right) .
$$

Since $A_{\lambda}(x)$ is 1-periodic, Floquet theory shows that

- the resolvent of the system can be written $S_{\lambda}(x) e^{x F_{\lambda}}$ where $S_{\lambda}$ is 1-periodic;

- since $A_{\lambda}$ depends continuously on $\lambda$, we have the same property for the resolvent $R_{\lambda}$, and the matrix $F_{\lambda}$ can also be chosen so that it depends continuously on $\lambda$ (this remark will be useful later; even though it is well known, we give an elementary proof of it in the Appendix (Section 5); see Lemma 5.10.

The Floquet exponents $\mu_{1}(\lambda), \mu_{2}(\lambda)$ are the eigenvalues of $F_{\lambda}$; since $F_{\lambda}$ depends continuously on $\lambda$, they also depend continuously on $\lambda$, and when $\mu_{1}(\lambda) \neq \mu_{2}(\lambda)$, a basis of $\mathbb{C}^{2}$ composed by eigenvectors of $F_{\lambda}$ generates a fundamental system of (4.5) (with $G=0$ ) of the form $\left(V_{i}(\lambda, \cdot)=\left(v_{i}(\lambda, \cdot), v_{i}^{\prime}(\lambda, \cdot)\right)\right)_{i \in\{1,2\}}$, where the $v_{i}(\lambda, \cdot)$ are solutions of

$$
-v^{\prime \prime}-2 r_{c} v^{\prime}+a(x) v+\lambda v=0
$$

and are of the form

$$
v_{i}(\lambda, x)=w_{i}(\lambda, x) e^{\mu_{i}(\lambda) x},
$$

where the $w_{i}(\lambda, \cdot)$ are 1-periodic functions. Hence the solutions of (4.6) are of the form

$$
v(x)=C_{1} w_{1}(\lambda, x) e^{\mu_{1}(\lambda) x}+C_{2} w_{2}(\lambda, x) e^{\mu_{2}(\lambda) x} .
$$

At last, using the Wronskian, we see that

$$
\Re e\left(\mu_{1}(\lambda)\right)+\Re e\left(\mu_{2}(\lambda)\right)=-2 r_{c} .
$$

This implies:

Lemma 4.1. Assume that

$$
\Re e\left(\mu_{1}(\lambda)\right) \Re e\left(\mu_{2}(\lambda)\right)<0 .
$$

Then, given $q_{0} \in B U C(\mathbb{R})$, the second-order differential equation (4.4) has one and only one bounded solution.

We leave the proof of Lemma 4.1 to the reader. It follows by writing the solutions $v$ of (4.4) using the basis $v_{1}, v_{2}$ of the homogeneous equation and the method of variation of constants.

Now, we need to know a precise localization of the spectrum of $L$; this is related to the values of $\lambda$ for which the assumption $\Re e\left(\mu_{1}(\lambda)\right) \Re e\left(\mu_{2}(\lambda)\right)<0$ will be satisfied, which will help us to find an integral formula for $v(\lambda, \cdot)=(\lambda I+L)^{-1} q_{0}$. 
4.2. The spectrum of $L$ in $B U C(\mathbb{R})$. Since $L$ is sectorial in $B U C(\mathbb{R})$ (see [29]), we know that there is some $a \in \mathbb{R}$ and $\varphi \in\left(0, \frac{\pi}{2}\right)$ such that the sector $S_{a, \varphi}=\{\lambda, \varphi \leq$ $|\arg (\lambda-a)| \leq \pi, \lambda \neq a\}$ is in the resolvent set of $A$, and $\|(\lambda I-L)\| \leq \frac{M}{|\lambda-a|}$ for all $\lambda \in S_{a, \varphi}$. There is nothing really original in this section; see [28, [5, [11. However the chain of arguments is, once again, nontrivial. We are going to localize more precisely the spectrum of $L$, and the goal of this subsection is to prove the following.

Proposition 4.2. Let $\lambda \in \sigma(-L)$. Then $\lambda=0$ or $\Re e(\lambda)<0$.

First, note that 0 is an eigenvalue of $L$ : indeed, $\psi_{r_{c}}$ satisfies $L \psi_{r_{c}}=0$. Another independent solution of the equation

$$
L v=-v^{\prime \prime}-2 r_{c} v^{\prime}+a(x) v=0
$$

is given by

$$
\psi_{r_{c}}(x) \int_{x}^{+\infty} \frac{e^{-2 r_{c} y}}{\psi_{r_{c}}^{2}(y)} d y
$$

The proof of Proposition 4.2 is now divided into several steps.

4.2.1. A link between the eigenvalues of $L$ and the Floquet exponents. As almost noted before, if $\Re e\left(\mu_{1}(\lambda)\right) \Re e\left(\mu_{2}(\lambda)\right)<0$, then $\lambda$ cannot be an eigenvalue of $-L$. Indeed, if $(\lambda I+L) v=0$, then $v$ is given by (4.8), and the boundedness of $v$ implies that the two constants $C_{i}$ are equal to 0 .

Hence the condition $\Re e\left(\mu_{1}(\lambda)\right) \Re e\left(\mu_{2}(\lambda)\right)<0$ is important to localize the spectrum of $-L$. A first step to know where the condition $\Re e\left(\mu_{1}(\lambda)\right) \Re e\left(\mu_{2}(\lambda)\right)<0$ is satisfied is the following.

Lemma 4.3. For all $\lambda \neq 0$ such that $\Re e \lambda \geq 0$, we have $\Re e \mu_{i}(\lambda) \neq 0$.

This will not yet allow us to know if $\Re e\left(\mu_{1}(\lambda)\right) \Re e\left(\mu_{2}(\lambda)\right)<0$ for all $\lambda \neq 0$ such that $\Re e \lambda \geq 0$, but then it will be sufficient to know that $\Re e\left(\mu_{1}(\lambda)\right) \Re e\left(\mu_{2}(\lambda)\right)<0$ at just some specific value of $\lambda$.

Proof of Lemma 4.3. The proof follows the scheme of [5]. We argue by contradiction, assuming that there exists a $\bar{\lambda}$ such that $\Re e \mu_{1}(\bar{\lambda})=0$. So the function $U_{1}(\bar{\lambda}, x)=w_{1}(\bar{\lambda}, x) e^{\mu_{1}(\bar{\lambda}) x}$ is bounded and satisfies the equation $L u+\bar{\lambda} u=0$. We assume in the sequel that $\Re e U_{1}(\bar{\lambda}, x) \neq 0$; otherwise we replace $U_{1}$ by $i U_{1}$. Consider the initial value problem

$$
\left\{\begin{array}{l}
v_{t}+L v=0, \\
v(0, x)=\Re e U_{1}(\bar{\lambda}, x) .
\end{array}\right.
$$

The solution of this equation is $v(t, x)=\Re e\left(e^{\bar{\lambda} t} U_{1}(\bar{\lambda}, x)\right)$. Since $U_{1}(\bar{\lambda}, x)$ is bounded and $\psi_{r_{c}}>0$, there exists $C>0$ such that

$$
\forall x \in \mathbb{R}, \quad-C \psi_{r_{c}}(x) \leq \Re e U_{1}(\bar{\lambda}, x) \leq C \psi_{r_{c}}(x) .
$$

We then distinguish the two cases $\Re e \bar{\lambda}>0$ and $\Re e \bar{\lambda}=0$.

The case $\Re e \bar{\lambda}>0$ : note that $\psi_{r_{c}}$ is a stationary solution of $v_{t}+L v=0$. Hence, using the maximum principle, we have that

$$
\forall t \geq 0, \forall x \in \mathbb{R}, \quad-C \psi_{r_{c}}(x) \leq \Re e\left(e^{\bar{\lambda} t} U_{1}(\bar{\lambda}, x)\right) \leq C \psi_{r_{c}}(x) .
$$

Since $\Re e \bar{\lambda}>0$, we get a contradiction by sending $t \rightarrow+\infty$. 
The case $\Re e \bar{\lambda}=0$ : we set $\bar{\lambda}=i \bar{\sigma}$ with $\bar{\sigma} \neq 0$. We assume, up to replacing $U_{1}(\bar{\lambda}, x)$ by $-U_{1}(\bar{\lambda}, x)$, that

$$
\zeta_{0}:=\inf \left\{\zeta>0, \Re e U_{1}(\bar{\lambda}, \cdot) \leq \zeta \psi_{r_{c}}\right\}>0 .
$$

We distinguish another two cases:

(ii') Assume that there exists $x_{0}$ such that $\Re e U_{1}\left(\bar{\lambda}, x_{0}\right)=\zeta_{0} \psi_{r_{c}}\left(x_{0}\right)$. Then the strong maximum principle implies that

$$
\Re e\left(e^{i \bar{\sigma} t} U_{1}(\bar{\lambda}, x)\right)=\zeta_{0} \psi_{r_{c}}(x) .
$$

Then, by denoting $U_{1}=u+i v$, we have on the one hand,

$$
\Re e\left(e^{i \bar{\sigma} t} U_{1}(\bar{\lambda}, x)\right)=\Re e((u+i v)(\cos (\bar{\sigma} t)+i \sin (\bar{\sigma} t)))=u \cos (\bar{\sigma} t)-v \sin (\bar{\sigma} t) .
$$

On the other hand, we have the equality at $t=0$,

$$
\Re e\left(U_{1}(\bar{\lambda}, x)\right)=u=\zeta_{0} \psi_{r_{c}}(x) .
$$

We deduce that for all $t>0$, we have

$$
u \cos (\bar{\sigma} t)-v \sin (\bar{\sigma} t)=u
$$

this means that

$$
u \frac{\cos (\bar{\sigma} t)-1}{t}=v \frac{\sin (\bar{\sigma} t)}{t} .
$$

By passing to the limit $t \rightarrow 0$, we get $\bar{\sigma} v=0$, so $v=u=0$, i.e. $U_{1}=0$, which is absurd.

$\left(\mathrm{ii}^{\prime \prime}\right)$ There exists $\left|x_{n}\right| \rightarrow+\infty$ such that $\Re e U_{1}\left(\bar{\lambda}, x_{n}\right)-\zeta_{0} \psi_{r_{c}}\left(x_{n}\right) \rightarrow 0$ as $n \rightarrow+\infty$. We bring back this case to the previous one as follows. We consider the Cauchy problem

$$
\left\{\begin{array}{l}
v_{t}^{n}-v_{x x}^{n}-2 r_{c} v_{x}^{n}+a_{n}(x) v^{n}=0 \\
v^{n}(0, x)=\Re e u_{n}(x),
\end{array}\right.
$$

with $a_{n}(x)=a\left(x+x_{n}\right)$ and $u_{n}(x)=U_{1}\left(\bar{\lambda}, x+x_{n}\right)$. The solution is $v^{n}(t, x)=$ $\Re e\left(e^{\bar{\lambda} t} u_{n}(x)\right)$. Since the sequence of functions $a_{n}$ is bounded in $C^{1}$, we can extract from the Ascoli theorem and the diagonal extraction process a subsequence which converges uniformly on any compact set. We denote by $a_{\infty}$ the limit function which is 1-periodic. We proceed in the same way for the sequence $u_{n}(x)$ which converges to $u_{\infty}(x)$ and the sequence $\psi_{r_{c}}\left(x+x_{n}\right)$ which converges to $\psi_{\infty}(x)$. From parabolic regularity, we can extract a subsequence of $v^{n}$ which converges locally in $C^{1,2}$ to the solution $v$ of the equation

$$
\left\{\begin{array}{l}
v_{t}-v_{x x}-2 r_{c} v_{x}+a_{\infty}(x) v=0 \\
v(0, x)=\Re e u_{\infty}(x)
\end{array}\right.
$$

which is $v(t, x)=\Re e\left(e^{\bar{\lambda} t} u_{\infty}(x)\right)$. Moreover, since $\Re e U_{1}\left(\bar{\lambda}, x_{n}\right)-\zeta_{0} \psi_{r_{c}}\left(x_{n}\right) \rightarrow 0$, we get that $\Re e u_{\infty}(0)=\zeta_{0} \psi_{\infty}(0)$. We are thus brought back to the case (ii').

Hence in any case we get a contradiction, which implies that for all $\lambda \neq 0$ such that $\Re e \lambda \geq 0$, we have $\Re e \mu_{i}(\lambda) \neq 0$. Lemma 4.3 is proved. 0 .

Now it remains to exhibit at least one value of $\lambda$ for which $\Re e\left(\mu_{1}(\lambda)\right) \Re e\left(\mu_{2}(\lambda)\right)<$ 
4.2.2. The spectrum of $L$ near 0 . First we use the fact that we know the eigenfunctions of $L$ associated to the eigenvalue 0 to determine a basis of solutions of (4.6) for $\lambda$ close to 0 :

Lemma 4.4. For $\lambda$ close to 0 , equation (4.6) has a solution $u_{1}(\lambda, x)$ which has the following (uniform in $x$ ) asymptotic expansion as $\lambda \rightarrow 0$ :

$$
u_{1}(\lambda, x)=\psi_{r_{c}}(x) e^{\int_{0}^{x}\left(\lambda \varphi_{1}-\lambda^{2} \varphi_{2}+O\left(\lambda^{3}\right)\right)},
$$

where $\varphi_{1}$ and $\varphi_{2}$ are the following positive and 1-periodic functions:

$$
\varphi_{2}(x)=\int_{-\infty}^{x} \frac{\psi_{r_{c}}^{2}(y)}{\psi_{r_{c}}^{2}(x)} \varphi_{1}^{2}(y) e^{-2 r_{c}(x-y)} d y=\int_{-\infty}^{0} \frac{\psi_{r_{c}}^{2}(z+x)}{\psi_{r_{c}}^{2}(x)} \varphi_{1}^{2}(z+x) e^{2 r_{c} z} d z .
$$

Another element of the fundamental system is given by

$$
u_{2}(\lambda, x)=u_{1}(\lambda, x) \int_{x}^{+\infty} \frac{e^{-2 r_{c} y}}{u_{1}^{2}(\lambda, y)} d y
$$

The proof is classical and will be omitted.

Lemma 4.5. For all $\lambda \neq 0$ close enough to 0 , and such that $\Re e \lambda \geq 0$, we have $\Re e\left(\mu_{1}(\lambda)\right) \Re e\left(\mu_{2}(\lambda)\right)<0$.

Proof of Lemma 4.5. We already have some information: the real parts $\Re e\left(\mu_{i}(\lambda)\right)$ are never equal to 0 in $\{\lambda \neq 0, \Re e \lambda \geq 0\}$; moreover their sum is constant equal to $-2 r_{c}$, hence negative. Therefore the real parts $\Re e\left(\mu_{i}(\lambda)\right)$ remain either positive or negative, but both cannot be positive. Hence, there are only two possible cases:

- either both of them are negative,

- or one of them is positive and the other is negative.

Lemma 4.5 states that we are in the second case. This follows from the asymptotic development obtained in Lemma 4.4; indeed, if both real parts $\Re e\left(\mu_{i}(\lambda)\right)$ are negative, then it follows from (4.8) that all the solutions of (4.6), in particular $u_{1}$, go to 0 as $x \rightarrow+\infty$. However, it follows from (4.10) that

$$
\left|u_{1}(\lambda, x)\right|=\psi_{r_{c}}(x) \exp e^{\int_{0}^{x} \Re e\left(\lambda \varphi_{1}-\lambda^{2} \varphi_{2}+O\left(\lambda^{3}\right)\right)},
$$

and if $\lambda=\lambda_{1}+i \lambda_{2}$, we have

$$
\Re e\left(\lambda \varphi_{1}-\lambda^{2} \varphi_{2}\right)=\lambda_{1} \varphi_{1}+\left(\lambda_{2}^{2}-\lambda_{1}^{2}\right) \varphi_{2} ;
$$

hence, since $\varphi_{1}$ and $\varphi_{2}$ are positive and 1-periodic, if $\lambda_{1}>0$ or if $\lambda_{1}=0$ and $\lambda_{2} \neq 0$, the function $\Re e\left(\lambda \varphi_{1}-\lambda^{2} \varphi_{2}\right)$ is bounded from below by a positive constant if $|\lambda|$ is small enough. Hence the same occurs for $\Re e\left(\lambda \varphi_{1}-\lambda^{2} \varphi_{2}+O\left(\lambda^{3}\right)\right)$, and this implies that $\left|u_{1}(\lambda, x)\right| \rightarrow+\infty$ as $x \rightarrow+\infty$, in contradiction with the assumption that the real parts $\Re e\left(\mu_{i}(\lambda)\right)$ are both negative. Hence we are in the second case.

4.2.3. Conclusion: Localization of the spectrum and integral expression for $(\lambda I+$ $L)^{-1} q_{0}$. Now, if $\Sigma$ is the sector bypassing $\sigma(-L)$, we wish to prove the

Lemma 4.6. In the two cases: (i) $\lambda \neq 0$ such that $\Re e(\lambda) \geq 0$, (ii) $\Re e(\lambda)<0$ and $\lambda \notin \Sigma$, we have

$$
\Re e\left(\mu_{1}(\lambda)\right) \Re e\left(\mu_{2}(\lambda)\right)<0 .
$$


As a consequence, we immediately obtain

Corollary 4.7. Given $q_{0} \in B U C(\mathbb{R})$, there exists a unique bounded solution of (4.4); moreover, if additionally $\lambda$ is close enough to 0 , it is given by the relation

$$
v(\lambda, x)=u_{1}(\lambda, x) \int_{x}^{+\infty} u_{2}(\lambda, y) q_{0}(y) e^{2 r_{c} y} d y+u_{2}(\lambda, x) \int_{-\infty}^{x} u_{1}(\lambda, y) q_{0}(y) e^{2 r_{c} y} d y,
$$

where $u_{1}$ and $u_{2}$ are given by (4.10) and (4.13).

Note that the formula (4.14) is obtained by using the basis $u_{1}(\lambda, x)$ and $u_{2}(\lambda, x)$ given by Lemma 4.4, and the method of variation of constants. Hence it remains to prove Lemma 4.6 .

Proof of Lemma 4.6. Combining Lemmas 4.3 and 4.5. we already know that $\Re e\left(\mu_{1}(\lambda)\right) \Re e\left(\mu_{2}(\lambda)\right)<0$ for all $\lambda \neq 0$ such that $\Re e(\lambda) \geq 0$. Now we want to prove that this property also holds for all $\lambda \notin \Sigma$ such that $\Re e(\lambda)<0$. We can assume that $\Re e\left(\mu_{1}\right)$ is positive on $\{\Re e \lambda>0\}$, and then it is sufficient to prove that it remains positive on $\{\lambda \notin \Sigma, \Re e(\lambda)<0\}$, since then $\Re e\left(\mu_{2}(\lambda)\right)=-2 r_{c}-\Re e\left(\mu_{1}(\lambda)\right)<0$. By contradiction, assume that there is some $\lambda_{1} \notin \Sigma, \Re e \lambda_{1}<0$, such that $\Re e\left(\mu_{1}\left(\lambda_{1}\right)\right) \leq$ 0 . Then

- if $\Re e\left(\mu_{1}\left(\lambda_{1}\right)\right)=0$, then there is some nonzero bounded solution of $\left(\lambda_{1} I+\right.$ $L) v=0$ (remember (4.7)), which implies that $\lambda_{1}$ is an eigenvalue of $-L$, which is a contradiction with the assumption $\lambda_{1} \notin \Sigma$;

- if $\Re e\left(\mu_{1}\left(\lambda_{1}\right)\right)<0$, choose $\lambda_{2}:=i \Im m \lambda_{1}$; then $\Re e\left(\mu_{1}\left(\lambda_{2}\right)\right)>0$, and since $\Re e\left(\mu_{1}\right)$ is continuous on $\lambda, \Re e\left(\mu_{1}\right)$ has to vanish somewhere on $\left[\lambda_{1}, \lambda_{2}\right]$, which is impossible, as we have seen before.

Hence, $\Re e\left(\mu_{1}(\lambda)\right)>0$ for all $\lambda \notin \Sigma, \Re e(\lambda)<0$, and consequently $\Re e\left(\mu_{2}(\lambda)\right)<$ $-2 r_{c}$.

4.3. Application: Another integral formula for $s$. Remember that the HopfCole transform $q$ of the shift $m$ is given by (4.3). We are going to transform this formula in a more useful form:

Lemma 4.8. Denote

$$
\gamma_{\varepsilon}:=(-i \infty,-i \varepsilon] \cup C_{\varepsilon} \cup[i \varepsilon, i \infty),
$$

where $C_{\varepsilon}:=\left\{z=\varepsilon e^{i \theta}, \theta \in\left[-\frac{\pi}{2}, \frac{\pi}{2}\right]\right\}$. Then

$$
q(t, x)=\frac{1}{2 i \pi} \int_{\gamma_{\varepsilon}} e^{t \lambda}(\lambda I+L)^{-1} q_{0} d \lambda .
$$

Proof of Lemma 4.8, Let us denote $\Sigma^{\prime}:=\{\lambda \neq 0, \Re e \lambda \geq 0\} \cup\{\lambda \notin \Sigma, \Re e \lambda<0\}$. We have seen that $\lambda I+L$ is invertible for all $\lambda \in \Sigma^{\prime}$. Moreover, $\Re e \mu_{2}(\lambda)<-2 r_{c}<$ $0<\Re e \mu_{1}(\lambda)$ for all $\lambda \in \Sigma^{\prime}$; since $\mu_{1}$ and $\mu_{2}$ are continuous, there exists an open neighborhood $\Sigma^{\prime \prime}$ of $\Sigma^{\prime}$ (hence that contains $i \mathbb{R}^{*}$ ) such that $\Re e \mu_{2}(\lambda)<-2 r_{c}<0<$ $\Re e \mu_{1}(\lambda)$ remains true for all $\lambda \in \Sigma^{\prime \prime}$, which implies that $\lambda I+L$ remains invertible in $\Sigma^{\prime \prime}$, hence that $(\lambda I+L)^{-1}$ is holomorphic in $\Sigma^{\prime \prime}$.

This allows us to obtain another expression of the Hopf-Cole transform $q$ of the shift $m$. Indeed, consider (see Figure 2):

$$
\gamma_{\delta, \varepsilon}:=D_{\delta}^{-} \cup[-i \delta,-i \varepsilon] \cup C_{\varepsilon} \cup[i \varepsilon, i \delta] \cup D_{\delta}^{+},
$$




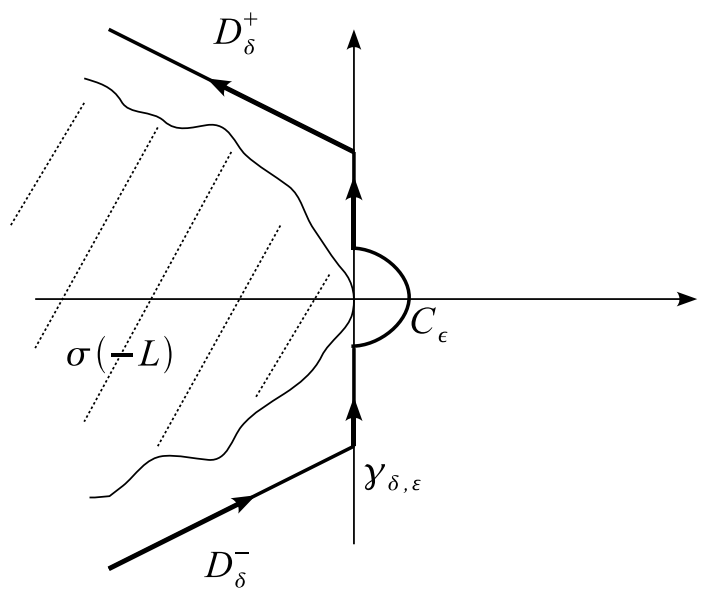

FiguRE 2. Integration contour $\gamma_{\delta, \varepsilon}$

with

$$
D_{\delta}^{-}=\{-i \delta+t(-1-\nu i), t \geq 0\}, \quad D_{\delta}^{+}=\{i \delta+t(-1+\nu i), t \geq 0\}
$$

the value $\nu>0$ is such that this path bypasses the spectrum and $\varepsilon$ is a quantity intended to tend to 0 .

Then $\gamma_{\delta, \varepsilon}$ is homotopic to the path $\gamma$ in $\Sigma^{\prime \prime}$, and the Cauchy theorem allows us to see that

$$
q(t, x)=\frac{1}{2 i \pi} \int_{\gamma} e^{t \lambda}(\lambda I+L)^{-1} q_{0} d l=\frac{1}{2 i \pi} \int_{\gamma_{\delta, \varepsilon}} e^{t \lambda}(\lambda I+L)^{-1} q_{0} d \lambda .
$$

But now, given $R>\delta$, denote by $z_{R}$ the affix of the intersection between the horizontal line passing through $i R$ and $D_{\delta}^{+}$: since $L$ is sectorial, we have

$$
\left\|(\lambda I+L)^{-1} q_{0}\right\|_{L^{\infty}(\mathbb{R})} \leq \frac{M}{|\lambda-a|}\left\|q_{0}\right\|_{L^{\infty}(\mathbb{R})} \leq \frac{M}{R}\left\|q_{0}\right\|_{L^{\infty}(\mathbb{R})} ;
$$

hence

$$
\int_{\left[i R, z_{R}\right]} e^{t \lambda}(\lambda I+L)^{-1} q_{0} d \lambda=O(\delta) \quad \text { as } R \rightarrow+\infty
$$

The same property holds for $D_{\delta}^{-}$. Sending $\delta$ to 0 , the Cauchy theorem implies (4.15).

\subsection{Approximation of the Hopf-Cole transform of the shift.}

4.4.1. A somewhat simplified expression of $v(\lambda, x)$ and $q$. In order to study the asymptotic properties of the expression of $q$ given by (4.15), we are going to simplify as much as possible the integrand $e^{t \lambda} v(\lambda, x)$. Combining the expression (4.14) of $(\lambda I+L)^{-1} q_{0}$, and the relation (4.13) between $u_{1}$ and $u_{2}$, we obtain that, for $|\lambda|$ small enough,

$$
v(\lambda, x)=u_{1}(\lambda, x)\left(\int_{y=x}^{+\infty} \int_{z=y}^{+\infty}+\int_{y=-\infty}^{x} \int_{z=x}^{+\infty}\right) \frac{u_{1}(\lambda, y)}{u_{1}^{2}(\lambda, z)} q_{0}(y) e^{2 r_{c}(y-z)} d z d y .
$$


Now, since for $\lambda$ close to 0 , we have

$$
u_{1}(\lambda, x)=\psi_{r_{c}}(x) e^{\int_{0}^{x}\left(\lambda \varphi_{1}-\lambda^{2} \varphi_{2}+O\left(\lambda^{3}\right)\right)},
$$

we can "approximate" $u_{1}$ by its truncated expression

$$
u_{1, t c}(\lambda, x)=\psi_{r_{c}}(x) e^{\int_{0}^{x}\left(\lambda \varphi_{1}-\lambda^{2} \varphi_{2}\right)} .
$$

This engages us to consider the following quantity, which we hope will be a good approximation of $v(x, \lambda)$ : for all $x \in \mathbb{R}$, all $\lambda \neq 0, \Re e \lambda \geq 0$,

$v_{t c}(\lambda, x):=u_{1, t c}(\lambda, x)\left(\int_{y=x}^{+\infty} \int_{z=y}^{+\infty}+\int_{y=-\infty}^{x} \int_{z=x}^{+\infty}\right) \frac{u_{1, t c}(\lambda, y)}{u_{1, t c}^{2}(\lambda, z)} q_{0}(y) e^{2 r_{c}(y-z)} d z d y$,

and the related quantities

$$
q_{\varepsilon}^{a p p}(t, x):=\frac{1}{2 i \pi} \int_{\gamma_{\varepsilon}} e^{t \lambda} v_{t c}(\lambda, x) d \lambda
$$

and

$$
q^{a p p}(t, x):=q_{0}^{a p p}(t, x)=\frac{1}{2 i \pi} \int_{i \mathbb{R}} e^{t \lambda} v_{t c}(\lambda, x) d l=\frac{1}{2 \pi} \int_{-\infty}^{+\infty} e^{i t \omega} v_{t c}(i \omega, x) d \omega .
$$

Our hope is that

- $v_{t c}$ will be a good approximation of $v=(\lambda I+L)^{-1} q_{0}$ for $\lambda$ close to 0 ,

- the behavior of $q$ is governed by the behavior of its integrand $e^{t \lambda}(\lambda I+L)^{-1} q_{0}$ near $l=0$, and hence $q^{a p p}$ will be a good approximation of $q$.

Then we will study $q^{a p p}$, in order to know some asymptotic properties of $q$.

First, we give the complete expression of $v_{t c}$ : introducing the functions $A_{1}(x, y, z)$ and $A_{2}(x, y, z)$ defined by

$$
A_{1}(x, y, z)=\int_{x}^{z} \varphi_{1}+\int_{y}^{z} \varphi_{1}, \quad A_{2}(x, y, z)=\int_{x}^{z} \varphi_{2}+\int_{y}^{z} \varphi_{2},
$$

we derive the following expression:

$$
\begin{aligned}
v_{t c}(\lambda, x)=\psi_{r_{c}}(x)\left(\int_{y=x}^{+\infty} \int_{z=y}^{+\infty}+\int_{y=-\infty}^{x} \int_{z=x}^{+\infty}\right) \\
\frac{q_{0}(y) \psi_{r_{c}}(y)}{\psi_{r_{c}}^{2}(z)} e^{-\lambda A_{1}+\lambda^{2} A_{2}} e^{2 r_{c}(y-z)} d z d y .
\end{aligned}
$$

4.4.2. Convergence results. For integrability reasons, we are going to consider the difference between the second time derivatives of $q$ and $q^{a p p}$, and we are going to prove the following.

\section{Lemma 4.9.}

$$
\left\|q_{t t}(t, \cdot)-q_{t t}^{a p p}(t, \cdot)\right\|_{L^{\infty}(\mathbb{R})}=O\left(\frac{1}{t}\right) .
$$

This will be sufficient for our purposes.

Proof of Lemma 4.9. The proof follows from the following claims:

Claim 4.10.

$q_{t t}(t, x)=\frac{1}{2 i \pi} \int_{\gamma_{\delta, \varepsilon}} \lambda^{2} e^{t \lambda}(\lambda I+L)^{-1} q_{0} d \lambda=\frac{1}{2 i \pi} \int_{-i \delta}^{i \delta} \lambda^{2} e^{t \lambda}(\lambda I+L)^{-1} q_{0} d \lambda+O\left(\frac{1}{t}\right)$. 
Claim 4.11.

$$
q_{t t}^{a p p}(t, x)=\frac{1}{2 i \pi} \int_{i \mathbb{R}} \lambda^{2} e^{t \lambda} v_{t c}(\lambda, x) d l=\frac{1}{2 i \pi} \int_{-i \delta}^{i \delta} \lambda^{2} e^{t \lambda} v_{t c}(\lambda, x) d \lambda+O\left(\frac{1}{t}\right) .
$$

Claim 4.12.

$$
\frac{1}{2 i \pi} \int_{-i \delta}^{i \delta} \lambda^{2} e^{t \lambda}(\lambda I+L)^{-1} q_{0} d l-\frac{1}{2 i \pi} \int_{-i \delta}^{i \delta} \lambda^{2} e^{t \lambda} v_{t c}(\lambda, x) d l=O\left(\frac{1}{t}\right) .
$$

Let us give the ideas of the proofs of these claims:

To prove Claim 4.10, first we decompose the integral on $\gamma_{\delta, \varepsilon}$ into three integrals: $D_{\delta}^{-}, D_{\delta}^{+}$and $[-i \delta,-i \varepsilon] \cup C_{\varepsilon} \cup[i \varepsilon, i \delta]$. It is easy to see that the integrals on $D_{\delta}^{-}$and $D_{\delta}^{+}$are of the order $O\left(\frac{1}{t}\right)$; concerning the last term, the presence of $\lambda^{2}$ makes the integral $\int_{-i \delta}^{i \delta} \lambda^{2} e^{t \lambda}(\lambda I+L)^{-1} q_{0} d \lambda$ convergent, and

$\int_{[-i \delta,-i \varepsilon] \cup C_{\varepsilon} \cup[i \varepsilon, i \delta]} \lambda^{2} e^{t \lambda}(\lambda I+L)^{-1} q_{0} d l \rightarrow \int_{[-i \delta, i \delta]} \lambda^{2} e^{t \lambda}(\lambda I+L)^{-1} q_{0} d l \quad$ as $\varepsilon \rightarrow 0$, which implies that Claim 4.10 is true.

To prove Claim 4.11, we first note that since $\psi_{r_{c}}, \varphi_{1}$ and $\varphi_{2}$ are positive and 1-periodic, there exist positive constants $a_{0}<b_{0}$ such that

$$
a_{0}(2 z-y-x) \leq A_{1}(x, y, z), A_{2}(x, y, z) \leq b_{0}(2 z-y-x) .
$$

This implies that $\lambda^{2} e^{t \lambda} v_{t c}(\lambda, x)$ is bounded and integrable on $i \mathbb{R}$ and $\frac{d}{d \lambda}\left(\lambda^{2} v_{t c}(\lambda, x)\right)$ $\in L^{1}((-i \infty,-i \delta) \cup(i \delta,+i \infty))$; hence an integration by parts (as for the RiemannLebesgue lemma) shows that Claim 4.11 is true.

To prove Claim 4.12, we estimate the difference $v(\lambda, x)-v_{t c}(\lambda, x)$, and we immediately see that, near $\lambda=0$, we have $v(\lambda, x)-v_{t c}(\lambda, x)=O\left(\frac{1}{\lambda}\right)$. Then, as previously, an integration by parts (thanks to the additional $\lambda^{2}$ ) proves that Claim 4.12 is true.

\subsection{Study of $q_{t t}^{a p p}$.}

4.5.1. New expression of $q_{t t}^{a p p}$. We begin by proving the following.

Claim 4.13.

$$
\begin{aligned}
q_{t t}^{a p p}(t, x)= & \frac{-1}{2 \pi} \int_{\mathbb{R}} \omega^{2} e^{i t \omega} v_{t c}(i \omega, x) d \omega \\
= & \frac{\psi_{r_{c}}(x)}{\sqrt{2 \pi}}\left(\int_{y=x}^{+\infty} \int_{z=y}^{+\infty}+\int_{y=-\infty}^{x} \int_{z=x}^{+\infty}\right) \\
& \frac{q_{0}(y) \psi_{r_{c}}(y)}{\psi_{r_{c}}^{2}(z)} \frac{d^{2}}{d t^{2}}\left(\frac{e^{-\frac{\left(t-A_{1}(x, y, z)\right)^{2}}{4 A_{2}(x, y, z)}}}{\sqrt{2 A_{2}(x, y, z)}}\right) e^{2 r_{c}(y-z)} d z d y .
\end{aligned}
$$

The proof follows from the formula of $q_{t t}^{a p p}$ given in Claim 4.11, the formula of $v_{t c}$ given in (4.21), and the Fubini theorem: indeed, as can be easily checked, the function

$$
(\omega, y, z) \mapsto \omega^{2} \frac{q_{0}(y) \psi_{r_{c}}(y)}{\psi_{r_{c}}^{2}(z)} e^{-i \omega A_{1}-\omega^{2} A_{2}} e^{2 r_{c}(y-z)} e^{i \omega t}
$$


is integrable on the corresponding domains; hence we can integrate first in $\omega$, to obtain that

$$
\begin{aligned}
q_{t t}^{a p p}(t, x)=\frac{\psi_{r_{c}}(x)}{\sqrt{2 \pi}}\left(\int_{y=x}^{+\infty} \int_{z=y}^{+\infty}+\int_{y=-\infty}^{x} \int_{z=x}^{+\infty}\right) \\
\frac{q_{0}(y) \psi_{r_{c}}(y)}{\psi_{r_{c}}^{2}(z)}\left(\frac{1}{\sqrt{2 \pi}} \int_{\mathbb{R}}-\omega^{2} e^{i \omega t} e^{-i \omega A_{1}-\omega^{2} A_{2}} d \omega\right) e^{2 r_{c}(y-z)} d z d y
\end{aligned}
$$

Inside the parentheses appears a well-known Fourier inverse since, with the convention $\mathcal{F} g(\omega)=\frac{1}{\sqrt{2 \pi}} \int_{\mathbb{R}} e^{-i \omega t} g(t) d \omega$, we have

$$
\mathcal{F}\left(\frac{e^{-\frac{(t-a)^{2}}{4 b}}}{\sqrt{2 b}}\right)(\omega)=e^{-i a \omega-b \omega^{2}} \quad \text { and } \quad \mathcal{F}\left(\frac{d^{2}}{d t^{2}} \frac{e^{-\frac{(t-a)^{2}}{4 b}}}{\sqrt{2 b}}\right)(\omega)=-\omega^{2} e^{-i a \omega-b \omega^{2}}
$$

which implies (4.23) and Claim 4.13,

Claim 4.13 engages us to consider the function

$$
\begin{aligned}
W(t, x)=\frac{\psi_{r_{c}}(x)}{\sqrt{2 \pi}}\left(\int_{y=x}^{+\infty} \int_{z=y}^{+\infty}\right. & \left.+\int_{y=-\infty}^{x} \int_{z=x}^{+\infty}\right) \\
& \frac{q_{0}(y) \psi_{r_{c}}(y)}{\psi_{r_{c}}^{2}(z)} \frac{e^{-\frac{\left(t-A_{1}(x, y, z)\right)^{2}}{4 A_{2}(x, y, z)}}}{\sqrt{2 A_{2}(x, y, z)}} e^{2 r_{c}(y-z)} d z d y .
\end{aligned}
$$

Combining Lemma 4.9 and Claim 4.13 , we have

$$
q_{t t}=W_{t t}+O 00\left(\frac{1}{t}\right)
$$

hence it remains to study $W_{t t}$. To estimate it, we split $W$ in the sequel as

$$
W(t, x)=W_{1}(t, x)+W_{2}(t, x),
$$

with

$$
W_{1}(t, x)=\frac{\psi_{r_{c}}(x)}{\sqrt{2 \pi}} \int_{y=x}^{+\infty} \int_{z=y}^{+\infty} \frac{q_{0}(y) \psi_{r_{c}}(y)}{\psi_{r_{c}}^{2}(z)} \frac{e^{-\frac{\left(t-A_{1}(x, y, z)\right)^{2}}{4 A_{2}(x, y, z)}}}{\sqrt{2 A_{2}(x, y, z)}} e^{2 r_{c}(y-z)} d z d y
$$

and

$$
W_{2}(t, x)=\frac{\psi_{r_{c}}(x)}{\sqrt{2 \pi}} \int_{y=-\infty}^{x} \int_{z=x}^{+\infty} \frac{q_{0}(y) \psi_{r_{c}}(y)}{\psi_{r_{c}}^{2}(z)} \frac{e^{-\frac{\left(t-A_{1}(x, y, z)\right)^{2}}{4 A_{2}(x, y, z)}}}{\sqrt{2 A_{2}(x, y, z)}} e^{2 r_{c}(y-z)} d z d y
$$

The reason is that the two integrals behave differently for large time.

4.5.2. Large time behavior of $W_{1}$ and its time derivatives. To study $W_{1}$, we make the change of variables $z^{\prime}=z-y, y^{\prime}=y-x$. Therefore we get

$$
W_{1}(t, x)=\frac{\psi_{r_{c}}(x)}{\sqrt{2 \pi}} \int_{0}^{+\infty} \int_{0}^{+\infty} \frac{q_{0}(x+y) \psi_{r_{c}}(x+y)}{\psi_{r_{c}}^{2}(x+y+z)} \frac{e^{-\frac{\left(t-\tilde{A}_{1}(x, y, z)\right)^{2}}{4 \tilde{A}_{2}(x, y, z)}}}{\sqrt{2 \widetilde{A}_{2}(x, y, z)}} e^{-2 r_{c} z} d z d y
$$

with

$$
\widetilde{A}_{1}(x, y, z)=\int_{x}^{x+y+z} \varphi_{1}+\int_{x+y}^{x+y+z} \varphi_{1}, \quad \widetilde{A}_{2}(x, y, z)=\int_{x}^{x+y+z} \varphi_{2}+\int_{x+y}^{x+y+z} \varphi_{2} .
$$


We then make another change of variables:

$$
Y=y, \quad Z=\widetilde{A}_{1}(x, y, z)=\int_{x}^{x+y+z} \varphi_{1}+\int_{x+y}^{x+y+z} \varphi_{1} .
$$

Note that $\widetilde{A}_{1}$ is increasing with respect to $z$. We then choose $F$ a primitive of $\varphi_{1}$ so that $\widetilde{A}_{1}(x, y, z)=2 F(x+y+z)-F(x)-F(x+y)$. Thus, we set

$$
Z=2 F(x+y+z)-F(x)-F(x+y), \quad z=F^{-1}\left(\frac{1}{2}(Z+F(x)+F(x+y))\right)-x-y .
$$

Therefore,

$$
\frac{d z}{d Z}=\frac{1}{2 \varphi_{1}\left(F^{-1}(Z / 2+(F(x)+F(x+y)) / 2)\right)}=\frac{1}{2 \varphi_{1}(x+y+z)} .
$$

We get

$$
\begin{aligned}
W_{1}(t, x)=\frac{\psi_{r_{c}}(x)}{\sqrt{2 \pi}} \int_{0}^{+\infty} \int_{\widetilde{A}_{1}(x, y, 0)}^{+\infty} \frac{q_{0}(x+y) \psi_{r_{c}}(x+y)}{\psi_{r_{c}}^{2}(x+y+Z)} & \\
& \frac{e^{-2 r_{c} z}}{2 \varphi_{1}(x+y+z)} \sqrt{\frac{\widetilde{A}_{1}}{2 \widetilde{A}_{2}}} \frac{e^{-\frac{\widetilde{A}_{1}}{4 \tilde{A}_{2}} \frac{(t-Z)^{2}}{Z}}}{\sqrt{Z}} d y d Z .
\end{aligned}
$$

We then denote $\widetilde{c}(x, y, z)=\frac{\widetilde{A}_{1}(x, y, z)}{\widetilde{A}_{2}(x, y, z)}$, which is a function bounded from below and from above. By inverting the formula $\widetilde{A}_{1}(x, y, 0)=F(x+y)-F(x)$ into $y=F^{-1}(Z+F(x))-x$, we obtain

$$
W_{1}(t, x)=\int_{0}^{+\infty} V_{1}(x, Z) \frac{e^{-\widetilde{c}(x, y, Z) \frac{(t-Z)^{2}}{4 Z}}}{\sqrt{Z}} d Z
$$

with (keep in mind formula (4.27)):

$$
V_{1}(x, Z)=\frac{\psi_{r_{c}}(x)}{2 \sqrt{\pi}} \int_{0}^{F^{-1}(Z+F(x))-x} \frac{q_{0}(x+y) \psi_{r_{c}}(x+y)}{\psi_{r_{c}}^{2}(x+y+z)} \frac{e^{-2 r_{c} z}}{2 \varphi_{1}(x+y+z)} \sqrt{\tilde{c}} d y .
$$

Lemma 4.14. The function $V_{1}(x, Z)$ is bounded.

Proof. Since $q_{0}, \psi_{r_{c}}, \varphi_{1}$ and $\widetilde{c}$ are bounded, we have

$$
V_{1}(x, Z) \leq C \int_{0}^{F^{-1}(Z+F(x))-x} e^{2 r_{c}(x+y)} e^{-2 r_{c} F^{-1}\left(\frac{1}{2}(Z+F(x)+F(x+y))\right)} d y .
$$

The key point is that $\varphi_{1}$ is 1-periodic and so we can write it as $\varphi_{1}(x)=M+\varepsilon(x)$, its mean value plus a periodic function with zero mean value. Thus, $F(x)=$ $M x+g(x)$, with $g$ bounded. By inverting, we get that $F^{-1}$ can be written as $F^{-1}(y)=\frac{y}{M}+h(y)$, with $h$ bounded. Note then that

$$
\begin{aligned}
F^{-1}(Z+F(x))-x & =F^{-1}(Z+M x+g(x))-x \\
& =\frac{1}{M}(Z+M x+g(x))+h(Z+M x+g(x))-x \\
& =\frac{Z}{M}+H(x, Z),
\end{aligned}
$$


with $H$ bounded. In the same way, we have

$$
\begin{aligned}
F^{-1} & \left(\frac{1}{2}(Z+F(x)+F(x+y))\right) \\
= & F^{-1}\left(\frac{Z}{2}+\frac{1}{2}(M x+g(x))+\frac{1}{2}(M(x+y)+g(x+y))\right) \\
= & F^{-1}\left(\frac{Z}{2}+M x+\frac{M}{2} y+\frac{1}{2}(g(x)+g(x+y))\right) \\
= & \frac{1}{M}\left(\frac{Z}{2}+M x+\frac{M}{2} y+\frac{1}{2}(g(x)+g(x+y))\right) \\
& +h\left(\frac{Z}{2}+M x+\frac{M}{2} y+\frac{1}{2}(g(x)+g(x+y))\right) .
\end{aligned}
$$

So,

$$
F^{-1}\left(\frac{1}{2}(Z+F(x)+F(x+y))\right)=\frac{Z}{2 M}+x+\frac{y}{2}+G(x, y, Z),
$$

with $G$ bounded. Thus,

$$
e^{2 r_{c}(x+y)} e^{-2 r_{c} F^{-1}\left(\frac{1}{2}(Z+F(x)+F(x+y))\right)}=e^{r_{c} y} e^{-r_{c} Z / M} e^{-2 r_{c} G(x, y, Z)} \leq C e^{r_{c} y} e^{-r_{c} Z / M} .
$$

Finally,

$$
V_{1}(x, Z) \leq C e^{-r_{c} Z / M} \int_{0}^{Z / M+H(x, Z)} e^{r_{c} y} d y=O(1),
$$

so $V_{1}$ is bounded.

Corollary 4.15. The function $W_{1}$ is bounded. Moreover, as $t \rightarrow+\infty$, we have $\partial_{t} W_{1}(t, x)=O\left(\frac{1}{\sqrt{t}}\right)$ and $\partial_{t t} W_{1}(t, x)=O\left(\frac{1}{t}\right)$.

Proof. Since $V_{1}$ is bounded and $\widetilde{c} \geq m>0$, we have

$$
\left|W_{1}(t, x)\right| \leq C \int_{0}^{+\infty} \frac{e^{-m \frac{(t-Z)^{2}}{4 Z}}}{\sqrt{Z}} d Z .
$$

We deduce that the function $W_{1}$ is bounded from Lemma 5.2, which we also use for the time derivatives.

4.5.3. Large time behavior of $W_{2}$ and its time derivatives. To study $W_{2}$, we make the change of variables $z^{\prime}=z-x, y^{\prime}=y-x$ :

$$
W_{2}(t, x)=\frac{\psi_{r_{c}}(x)}{\sqrt{2 \pi}} \int_{-\infty}^{0} \int_{0}^{+\infty} \frac{q_{0}(x+y) \psi_{r_{c}}(x+y)}{\psi_{r_{c}}^{2}(x+z)} \frac{e^{-\frac{\left(t-\bar{A}_{1}(x, y, z)\right)^{2}}{4 \bar{A}_{2}(x, y, z)}}}{\sqrt{2 \bar{A}_{2}(x, y, z)}} e^{2 r_{c}(y-z)} d y d z
$$

with

$$
\bar{A}_{1}(x, y, z)=\int_{x}^{x+z} \varphi_{1}+\int_{x+y}^{x+z} \varphi_{1}, \quad \bar{A}_{2}(x, y, z)=\int_{x}^{x+z} \varphi_{2}+\int_{x+y}^{x+z} \varphi_{2} .
$$

We then make another change of variables: $Y=y, Z=\bar{A}_{1}(x, y, z)$. Using the primitive $F$ of $\varphi_{1}$, we have $\bar{A}_{1}(x, y, z)=2 F(x+z)-F(x)-F(x+y)$. We set

$$
Z=2 F(x+z)-F(x)-F(x+y), \quad z=F^{-1}\left(\frac{1}{2}(Z+F(x)+F(x+y))\right)-x .
$$

We have

$$
\frac{d z}{d Z}=\frac{1}{2 \varphi_{1}\left(F^{-1}(Z / 2+(F(x)+F(x+y)) / 2)\right)}=\frac{1}{2 \varphi_{1}(x+z)} .
$$


Therefore we get

$$
\begin{aligned}
W_{2}(t, x)=\frac{\psi_{r_{c}}(x)}{\sqrt{2 \pi}} \int_{-\infty}^{0} \int_{\bar{A}_{1}(x, y, 0)}^{+\infty} & \frac{q_{0}(x+y) \psi_{r_{c}}(x+y)}{\psi_{r_{c}}^{2}(x+z)} \\
& \frac{e^{2 r_{c}(y-z)}}{2 \varphi_{1}(x+z)} \sqrt{\frac{\bar{A}_{1}}{2 \bar{A}_{2}}} \frac{e^{-\frac{\bar{A}_{1}}{4 \bar{A}_{2}} \frac{(t-Z)^{2}}{Z}}}{\sqrt{Z}} d y d Z .
\end{aligned}
$$

We then denote $\bar{c}(x, y, z)=\frac{\bar{A}_{1}(x, y, z)}{\bar{A}_{2}(x, y, z)}$, which is bounded from below and from above. Thanks to the Fubini theorem, we write $W_{2}$ as

$$
W_{2}(t, x)=\int_{0}^{+\infty} V_{2}(x, Z) \frac{e^{-\bar{c}(x, y, Z) \frac{(t-Z)^{2}}{4 Z}}}{\sqrt{Z}} d Z
$$

with

$$
V_{2}(x, Z)=\frac{\psi_{r_{c}}(x)}{2 \sqrt{\pi}} \int_{F^{-1}(F(x)-Z)-x}^{0} \frac{q_{0}(x+y) \psi_{r_{c}}(x+y)}{\phi_{c}^{2}(x+z)} \frac{e^{2 r_{c}(x+y)} e^{-2 r_{c}(y-z)}}{2 \varphi_{1}(x+z)} \sqrt{\bar{c}} d y .
$$

Lemma 4.16. There exist $C, \alpha>0$ such that $\left|V_{2}(x, Z)\right| \leq C e^{-a Z}$.

Proof. Since $q_{0}, \psi_{r_{c}}, \varphi_{1}$ and $\bar{c}$ are bounded, we have

$$
V_{2}(x, Z) \leq C \int_{F^{-1}(F(x)-Z)-x}^{0} e^{2 r_{c}(x+y)} e^{-2 r_{c} F^{-1}\left(\frac{1}{2}(Z+F(x)+F(x+y))\right)} d y .
$$

We again take the same notation as for the study of $V_{1}$. Here, we get

$$
\begin{gathered}
F^{-1}(F(x)-Z)-x=\frac{Z}{M}+H(x, Z), \\
F^{-1}\left(\frac{1}{2}(Z+F(x)+F(x+y))\right)=\frac{Z}{2 M}+x+y / 2+G(x, y, Z),
\end{gathered}
$$

with $H$ and $G$ bounded. We have

$$
e^{2 r_{c}(x+y)} e^{-2 r_{c} F^{-1}\left(\frac{1}{2}(Z+F(x)+F(x+y))\right)} \leq C e^{r_{c} y} e^{-r_{c} Z / M} .
$$

Finally,

so

$$
V_{2}(x, Z) \leq C e^{-r_{c} Z / M} \int_{-Z / M+H(x, Z)}^{0} e^{r_{c} y} d y
$$

$$
V_{2}(x, Z) \leq C e^{-r_{c} Z / M}\left(1-e^{-r_{c} Z / M} e^{r_{c} H(x, Z)}\right) \leq C e^{-r_{c} Z / M}
$$

Corollary 4.17. There exists $C, a>0$ such that for $t \rightarrow+\infty, W_{2}(t, x), \partial_{t} W_{2}(t, x)$, $\partial_{t t} W_{2}(t, x)=O\left(e^{-a t}\right)$.

Proof. From the previous lemma and that $\bar{c} \geq m>0$, we have

$$
\left|W_{2}(t, x)\right| \leq C \int_{0}^{+\infty} e^{-a Z} \frac{e^{-m \frac{(t-Z)^{2}}{4 Z}}}{\sqrt{Z}} d Z .
$$

The estimate on $W_{2}$ results from Lemma 5.3. as well as the time derivatives. 
4.6. Proof of Theorem 1.4. Now we can conclude the proof of Theorem 1.4 The consequence of Corollaries 4.15 and 4.17 is that $q_{t t}=O\left(\frac{1}{t}\right)$. Since $q$ is bounded (by the weak maximum principle), we obtain that $q_{t}=O\left(\frac{1}{\sqrt{t}}\right)$. This already implies that the approximate shift $m$ satisfies: $m_{t}=O\left(\frac{1}{\sqrt{t}}\right), m_{t t}=O\left(\frac{1}{t}\right)$. But we also need to estimate the spatial derivatives, and in particular $m_{x}$. To do this, we come back to

$$
s(t, x)=\frac{q(t, x)}{\psi_{r_{c}}(x)}=e^{c r_{c} m(t, x)},
$$

and we are going to prove that $s_{x}=O\left(\frac{1}{\sqrt{t}}\right)$. Indeed, we have seen that $s$ is solution of

$$
s_{t}-s_{x x}-2\left(r_{c}+\frac{\psi_{r_{c}}^{\prime}(x)}{\psi_{r_{c}}(x)}\right) s_{x}=0 .
$$

Denote

$$
k_{r_{c}}(x):=2\left(r_{c}+\frac{\psi_{r_{c}}^{\prime}(x)}{\psi_{r_{c}}(x)}\right)
$$

and

$$
K_{r_{c}}(x):=\int_{0}^{x} k_{r_{c}}(y) d y=2 r_{c} x+2 \ln \psi_{r_{c}}(x)-2 \ln \psi_{r_{c}}(0) .
$$

Then $k_{r_{c}}$ is a 1-periodic function, and $s_{x}$ is a solution of the nonhomogeneous first-order differential equation $w^{\prime}+k_{r_{c}}(x) w=s_{t}$; hence

$$
s_{x}(t, x)=\left(s_{x}(t, 0)+\int_{0}^{x} e^{K_{r_{c}}(y)} s_{t}(t, y) d y\right) e^{-K_{r_{c}}(x)} .
$$

Since from parabolic estimates $x \mapsto s_{x}(t, \cdot)$ is bounded, then $s_{x}(t, x) e^{K_{r_{c}}(x)} \rightarrow 0$ as $x \rightarrow-\infty$; thus

We deduce that

$$
s_{x}(t, x)=\left(\int_{-\infty}^{x} e^{K_{r_{c}}(y)} s_{t}(t, y) d y\right) e^{-K_{r_{c}}(x)} .
$$

$$
\left|s_{x}(t, x)\right| \leq \frac{C}{\sqrt{t}}\left(\int_{-\infty}^{x} e^{K_{r_{c}}(y)} d y\right) e^{-K_{r_{c}}(x)} \leq \frac{C^{\prime}}{\sqrt{t}},
$$

which gives that $s_{x}=O\left(\frac{1}{\sqrt{t}}\right)$, and thus $m_{x}=O\left(\frac{1}{\sqrt{t}}\right)$. The equation on $s$ also gives that $s_{x x}=O\left(\frac{1}{\sqrt{t}}\right)$; hence $m_{x x}=O\left(\frac{1}{\sqrt{t}}\right)$. This completes the proof of Theorem 1.4 .

\section{Appendix: Technical lemmas}

\subsection{Continuity of the Floquet decomposition.}

Lemma 5.1. In the representation of the resolvent $R_{\lambda}$ of the differential problem (4.5) : $R_{\lambda}=S_{\lambda}(x) e^{x F_{\lambda}}$, the matrix $F_{\lambda}$ can be chosen so that it depends continuously on $\lambda$.

Proof of Lemma 5.1, $F_{\lambda}$ has to be chosen such that $e^{F_{\lambda}}=R_{\lambda}(1)$, which depends continuously on $\lambda$. We are going to prove that there exists such a matrix $F_{\lambda}$, depending continuously on $\lambda$ : first consider the eigenvalues $k_{1}(\lambda), k_{2}(\lambda)$ of $R_{\lambda}(1)$, and choose $\varepsilon_{1}(\lambda)$ to be an eigenvector of norm equal to 1 associated to $k_{1}(\lambda) ; k_{1}(\lambda)$ and $k_{2}(\lambda)$ are continuous with respect to $\lambda$, so $\varepsilon_{1}(\lambda)$ can also be chosen continuously with respect to $\lambda$. Now consider $\varepsilon_{2}(\lambda)$ obtained from the rotation of $\varepsilon_{1}(\lambda)$ by the 
rotation of angle $\frac{\pi}{2}$ and write the matrix $P(\lambda)=\left(\varepsilon_{1}(\lambda) e_{2}(\lambda)\right)$. Then there is a continuous function $b(\lambda)$ such that

$$
P(\lambda)^{-1} R_{\lambda}(1) P(\lambda)=\left(\begin{array}{cc}
k_{1}(\lambda) & b(\lambda) \\
0 & k_{2}(\lambda)
\end{array}\right)=k_{1}(\lambda)\left(\begin{array}{cc}
1 & b(\lambda) / k_{1}(\lambda) \\
0 & k_{2}(\lambda) / k_{1}(\lambda)
\end{array}\right) .
$$

Hence, it is sufficient to prove that there exists $G_{\lambda}$ depending continuously on $\lambda$ such that

$$
e^{G_{\lambda}}=\left(\begin{array}{cc}
1 & (\lambda) / k_{1}(\lambda) \\
0 & k_{2}(\lambda) / k_{1}(\lambda)
\end{array}\right)=:\left(\begin{array}{cc}
1 & \beta(\lambda) \\
0 & k(\lambda)
\end{array}\right) .
$$

Searching $G_{\lambda}$ under the form

$$
G_{\lambda}=\left(\begin{array}{cc}
0 & \gamma_{1}(\lambda) \\
0 & \gamma_{2}(\lambda)
\end{array}\right)
$$

we immediately obtain that $\gamma_{1}(\lambda)$ and $\gamma_{2}(\lambda)$ have to satisfy

$$
e^{\gamma_{2}(\lambda)}=k(\lambda), \quad \gamma_{1}(\lambda) \frac{e^{\gamma_{2}(\lambda)}-1}{\gamma_{2}(\lambda)}=\beta(\lambda) ;
$$

since $k(\lambda) \neq 0$ and the function $z \mapsto \frac{e^{z}-1}{z}$ has no root, it is clear that there exist continuous functions $\gamma_{1}$ and $\gamma_{2}$ satisfying these conditions. Hence Lemma 5.1 is proved.

5.2. Estimates of the Gaussian integrals (useful for $W_{1}$ and $W_{2}$ ). The study of the function $W$ relies on elementary, but tedious, integral estimates. Lemma 5.2 below is related to $W_{1}$ (defined by (4.25)), and Lemma 5.3 below is related to the function $W_{2}$ (defined by (4.26) ).

Lemma 5.2. Let $a>0, b>0$. We have the following estimates:

$$
\begin{aligned}
& I_{1}(t):=\int_{0}^{+\infty} \frac{e^{-a \frac{(t-x)^{2}}{x}}}{\sqrt{x}} d x \text { is bounded, } \\
& I_{2}(t):=\int_{0}^{+\infty}|t-x| \frac{e^{-a \frac{(t-x)^{2}}{x}}}{x^{3 / 2}} d x=O\left(\frac{1}{\sqrt{t}}\right), \\
& I_{3}(t):=\int_{0}^{+\infty}\left((t-x)^{2}+x\right) \frac{e^{-a \frac{(t-x)^{2}}{x}}}{x^{5 / 2}} d x=O\left(\frac{1}{t}\right) .
\end{aligned}
$$

Remark. The first integral is even constant and equal to $\sqrt{\frac{\pi}{a}}$.

Proof of Lemma 5.2. The idea to prove these estimates is to cut $\mathbb{R}^{+}$as $[0, t] \cup[t,+\infty[$ and to make the change of variables $u=\frac{(t-x)^{2}}{2 x}$. On the domain $[0, t]$ the change of variables is such that

$$
x=t+u-\sqrt{u^{2}+2 u t}, \quad d x=\left(\frac{t+u-\sqrt{u^{2}+2 u t}}{\sqrt{u^{2}+2 u t}}\right) d u .
$$

On $[t,+\infty]$, the change of variables is

$$
x=t+u+\sqrt{u^{2}+2 u t}, \quad d x=\left(\frac{t+u+\sqrt{u^{2}+2 u t}}{\sqrt{u^{2}+2 u t}}\right) d u .
$$


(i) Estimate of $I_{1}$. We have

$$
\begin{aligned}
I_{1}(t)= & \int_{0}^{+\infty} \frac{e^{-a \frac{(t-x)^{2}}{x}}}{\sqrt{x}} d x=\int_{0}^{t} \frac{e^{-a \frac{(t-x)^{2}}{x}}}{\sqrt{x}} d x+\int_{t}^{+\infty} \frac{e^{-a \frac{(t-x)^{2}}{x}}}{\sqrt{x}} d x \\
= & \int_{0}^{+\infty} \frac{\sqrt{t+u-\sqrt{u^{2}+2 u t}+\sqrt{t+u+\sqrt{u^{2}+2 u t}}}}{\sqrt{u^{2}+2 u t}} e^{-2 a u} d u \\
= & \int_{0}^{+\infty} \frac{\sqrt{1+u / t-\sqrt{u^{2} / t^{2}+2 u / t}}}{\sqrt{u^{2} / t+2 u}} e^{-2 a u} d u \\
& +\int_{0}^{+\infty} \frac{\sqrt{1+u / t+\sqrt{u^{2} / t^{2}+2 u / t}}}{\sqrt{u^{2} / t+2 u}} e^{-2 a u} d u \\
\leq & 2 \int_{0}^{+\infty} \frac{\sqrt{1+u+\sqrt{u^{2}+2 u}}}{\sqrt{2 u}} e^{-2 a u} d u \leq C,
\end{aligned}
$$

the last inequalities being true for all $t \geq 1$.

(ii) Estimate of $I_{2}$. We have

$$
\begin{gathered}
I_{2}(t)=\int_{0}^{+\infty}|t-x| \frac{e^{-a \frac{(t-x)^{2}}{x}}}{x^{3 / 2}} d x=\int_{0}^{t}|t-x| \frac{e^{-a \frac{(t-x)^{2}}{x}}}{x^{3 / 2}} d x+\int_{t}^{+\infty}|t-x| \frac{e^{-a \frac{(t-x)^{2}}{x}}}{x^{3 / 2}} d x \\
=\int_{0}^{+\infty} \frac{\sqrt{u^{2}+2 u t}-u}{\sqrt{u^{2}+2 u t} \sqrt{t+u-\sqrt{u^{2}+2 u t}}} e^{-2 a u} d u \\
\quad+\int_{0}^{+\infty} \frac{\sqrt{u^{2}+2 u t}+u}{\sqrt{u^{2}+2 u t} \sqrt{t+u+\sqrt{u^{2}+2 u t}}} e^{-2 a u} d u .
\end{gathered}
$$

We prove that the last two integrals behave as $O\left(\frac{1}{\sqrt{t}}\right)$. We detail the second case; the first is less direct due to the minus sign in the denominator, but we can bypass this difficulty thanks to the estimate

$$
1+u / t-\sqrt{u^{2} / t^{2}+2 u / t} \geq \frac{C}{1+u},
$$

valid for all $t \geq 1$. In the second integral, if we factor $t$ out, we get

$$
\frac{1}{\sqrt{t}} \int_{0}^{+\infty} \frac{\sqrt{u^{2} / t+2 u}+u / \sqrt{t}}{\sqrt{u^{2} / t+2 u} \sqrt{1+u / t+\sqrt{u^{2} / t^{2}+2 u}}} e^{-2 a u} d u
$$

where the remaining integral is bounded since for $t \geq 1$,

$$
\begin{aligned}
\int_{0}^{+\infty} \frac{\sqrt{u^{2} / t+2 u}+u / \sqrt{t}}{\sqrt{u^{2} / t+2 u} \sqrt{1+u 6 / t+\sqrt{u^{2} / t^{2}+2 u}}} e^{-2 a u} d u & \leq \int_{0}^{+\infty} \frac{\sqrt{u^{2}+2 u}+u}{\sqrt{2 u}} e^{-2 a u} d u \\
& \leq C .
\end{aligned}
$$

(iii) Estimate of $I_{3}$. The proof is similar. 
Lemma 5.3. Let $a>0, b>0$. Then there exists $d>0$ such that

$$
\begin{aligned}
& J_{1}(t):=\int_{0}^{+\infty} e^{-a x} \frac{e^{-b \frac{(t-x)^{2}}{x}}}{\sqrt{x}} d x=O\left(e^{-d t}\right), \\
& J_{2}(t):=\int_{0}^{+\infty}|t-x| e^{-a x} \frac{e^{-b \frac{(t-x)^{2}}{x}}}{x^{3 / 2}} d x=O\left(e^{-d t}\right), \\
& J_{3}(t):=\int_{0}^{+\infty}\left((t-x)^{2}+x\right) e^{-a x} \frac{e^{-b \frac{(t-x)^{2}}{x}}}{x^{5 / 2}} d x=O\left(e^{-d t}\right) .
\end{aligned}
$$

Remark. We can prove in fact that $J_{1}$ is equal to $\sqrt{\frac{\pi}{a+b}} e^{-2 \sqrt{b}(\sqrt{a+b}-\sqrt{b}) t}$.

Proof of Lemma 5.3. To prove the exponential behavior, we proceed as in the proof of the previous lemma. Here, we just detail the estimate on $J_{1}$, since there are no particular difficulties with the two other integrals.

We want to get the estimate

$$
J_{1}(t):=\int_{0}^{+\infty} e^{-a x} \frac{e^{-b \frac{(t-x)^{2}}{x}}}{\sqrt{x}} d x=O\left(e^{-d t}\right) .
$$

We make for that the change of variables $u=a x+b \frac{(t-x)^{2}}{x}$. Note that the function $x \mapsto a x+b \frac{(t-x)^{2}}{x}$ achieves its minimum at the point $x=\sqrt{\frac{b}{a+b}} t$, and the value of this minimum is $2 b t\left(\sqrt{\frac{a+b}{b}-1}\right)$. We cut the integral $J_{1}$ into two parts:

$$
J_{1}(t)=\int_{0}^{\sqrt{\frac{b}{a+b}} t} e^{-a x} \frac{e^{-b \frac{(t-x)^{2}}{x}}}{\sqrt{x}} d x+\int_{\sqrt{\frac{b}{a+b}} t}^{+\infty} e^{-a x} \frac{e^{-b \frac{(t-x)^{2}}{x}}}{\sqrt{x}} d x .
$$

For the integral on $\left[0, \sqrt{\frac{b}{a+b}} t\right]$, the change of variables can be rewritten as

$$
x=\frac{1}{2}\left(2 b t+u-\sqrt{u^{2}+4 b u t-4 a b t^{2}}\right), \quad \frac{d x}{d u}=\frac{1}{2}\left(1-\frac{u+2 b t}{\sqrt{u^{2}+4 b u t-4 a b t^{2}}}\right) .
$$

Thus,

$$
\begin{aligned}
& \int_{0}^{\sqrt{\frac{b}{a+b}} t} e^{-a x} \frac{e^{-b \frac{(t-x)^{2}}{x}}}{\sqrt{x}} d x \\
= & \int_{2 b t\left(\sqrt{\left.\frac{a+b}{b}-1\right)}+\infty\right.}\left(\frac{u+2 b t}{\sqrt{u^{2}+4 b u t-4 a b t^{2}}}-1\right) \frac{e^{-u} d u}{\sqrt{2}\left(2 b t u-\sqrt{u^{2}+4 b u t-4 a b t^{2}}\right)^{1 / 2}} .
\end{aligned}
$$


We then make a further change of variables $v=u-2 b t\left(\sqrt{\frac{a+b}{b}}-1\right)$. By setting $\alpha:=\frac{a}{b}>0 \quad \varepsilon:=\sqrt{1+\frac{a}{b}}-1>0$, we get $2 u+4 b t=2 v+4 b \sqrt{1+\alpha} t$. Thus,

$$
\begin{aligned}
& \int_{0}^{\sqrt{\frac{b}{a+b}} t} e^{-a x} \frac{e^{-b \frac{(t-x)^{2}}{x}}}{\sqrt{x}} d x \\
= & \left.\int_{0}^{+\infty}\left(\frac{v+4 b t \sqrt{1+\alpha}}{\sqrt{v(v+4 b t \sqrt{1+\alpha})}}-1\right) \frac{e^{-v} e^{-2 b \varepsilon t} d v}{\sqrt{2}(v+2 b t \sqrt{1+\alpha}-\sqrt{v(v+4 b t \sqrt{1+\alpha}})}\right)^{1 / 2}
\end{aligned}
$$

Then we factor out the exponential term $e^{-2 b \varepsilon t}$. The last step is to prove that the remaining integral is bounded. We have

$$
\begin{aligned}
& \int_{0}^{+\infty}\left(\frac{v+4 b t \sqrt{1+\alpha}}{\sqrt{v(v+4 b t \sqrt{1+\alpha})}}-1\right) \frac{e^{-v} d v}{\sqrt{2}(v+2 b t \sqrt{1+\alpha}-\sqrt{v(v+4 b t \sqrt{1+\alpha})})^{1 / 2}} \\
& =\int_{0}^{+\infty} \frac{(v+4 b t \sqrt{1+\alpha}-\sqrt{v(v+4 b t \sqrt{1+\alpha})}) e^{-v} d v}{\sqrt{2} \sqrt{v(v+4 b t \sqrt{1+\alpha})}(v+2 b t \sqrt{1+\alpha}-\sqrt{v(v+4 b t \sqrt{1+\alpha})})^{1 / 2}} \\
& =\int_{0}^{+\infty} \frac{t(v / t+4 b \sqrt{1+\alpha}-\sqrt{v / t(v / t+4 b \sqrt{1+\alpha})}) e^{-v} d v}{\sqrt{t} \sqrt{t} \sqrt{2} \sqrt{v(v / t+4 b \sqrt{1+\alpha})}(v / t+2 b \sqrt{1+\alpha}-\sqrt{v / t(v / t+4 b \sqrt{1+\alpha})})^{1 / 2}} .
\end{aligned}
$$

The previous integral is convergent at $v=0$, because of the estimate

$$
1+s-\sqrt{s^{2}+2 s} \geq \frac{C}{1+s} .
$$

Thus, the integral on $\left[0, \sqrt{\frac{b}{a+b}} t\right]$ decreases exponentially with respect to $t$. We then follow the same way for the integral on the domain $\left[\sqrt{\frac{b}{a+b}} t,+\infty\right]$ by using the change of variables

$$
x=\frac{1}{2}\left(2 b t+u+\sqrt{u^{2}+4 b u t-4 a b t^{2}}\right) .
$$

The estimates are direct here since there is no minus sign in the denominator.

\section{ACKNOWLEDGEMENTS}

The authors are indebted to Professors T. Gallay and F. Hamel for valuable comments on this work. A remark made by Professor T. Gallay during the Ph.D. thesis defense of the first author, pointing out that the important issue was to really understand the attraction dynamics of the family of waves, was influential in the preparation and orientation of this work. They are also grateful to Professor F. Hamel for making his work [14] accessible to them. At last, the second author thanks Professor P. Cannarsa and the University Roma Tor Vergata, for an invitation that was helpful in the completion of this work. 


\section{REFERENCES}

[1] M. BAGEs, P. MARTinez, Existence of pulsating waves for a thermo-diffusive system modelling solid combustion, preprint.

[2] M. Bages, P. Martinez, J.-M. Roquejoffre, Dynamique en grand temps pour une classe d'équations de type KPP en milieu périodique, C.R. Acad. Sci. Paris, Ser. I, 346 (2008), pp. 1051-1056. MR2462047(2009j:35296)

[3] H. Berestycki, F. Hamel, Front propagation in periodic excitable media, Commun. Pure Appl. Math., 55 (2002), pp. 949-1032. MR1900178 (2003d:35139)

[4] H. Berestycki, F. Hamel, Generalized travelling waves for reaction-diffusion equations, In: Perspectives in Nonlinear Partial Differential Equations. In honor of H. Brezis, Contemp. Math. 446, Amer. Math. Soc., pp. 101-123. MR2373726 (2009k:35135)

[5] H. Berestycki, B. Larrouturou, J.-M. Roquejoffre, Stability of travelling fronts in a curved flame model. Part I: Linear analysis, Arch. Rat. Mech. Anal., 117 (1991), pp. 97-117. MR:1082630 (91m:35114)

[6] H. Berestycki, L. Nirenberg, Travelling fronts in cylinders, Ann. Inst. H. Poincaré, Anal. non Linéaire, 9 (1992), pp. 497-572. MR1191008 (93k:35019)

[7] M. Bramson, Convergence of solutions of the Kolmogorov equation to travelling waves, Memoirs of the AMS, 44 (1983), Providence, R.I. MR705746 (84m:60098)

[8] P. Collet, J.P. Eckmann, Space-time behaviour in problems of hydrodynamic type: a case study, Nonlinearity 5 (1992), no. 6, pp. 1265-1302. MR.1192518(94d:58131)

[9] P. Constantin, K. Domelevo, J.-M. Roquejoffre, L. RyzhiK, Existence of pulsating waves in a model of flames in sprays, Journal of the European Mathematical Society, 8 (2006), pp. 555-584. MR2262195 (2007g:35084)

[10] U. Ebert, W. van SAarloos, Front propagation into unstable states: universal algebraic convergence towards uniformly translating pulled fronts, Physica D 146 (2000), pp. 1-99. MR.1787406 (2002e:35113)

[11] R.A. Gardner, On the structure of the spectra of periodic travelling waves, J. Math. Pures Appl. 72 (1993), pp. 415-439. MR.1239098 (94j:35072)

[12] F. HAMEL, Qualitative properties of monostable pulsating fronts: exponential decay and monotonicity, J. Math. Pures Appl. 89 (2008), pp. 355-399. MR2401143 (2009g:35132)

[13] F. Hamel, N. Nadirashvili, Travelling fronts and entire solutions of the Fisher-KPP equation in $\mathbb{R}^{N}$, Arch. Ration. Mech. Anal. 157 (2001), pp. 91-163. MR.1830037 (2002d:35090)

[14] F. Hamel, L. Roques, Uniqueness and stability properties of monostable pulsating fronts, J. European Math. Soc. 13 (2011), pp. 345-390. MR.2746770

[15] F. Hamel, L. RYZhik, Non-adiabatic KPP fronts with an arbitrary Lewis number, Nonlinearity 18 (2005), pp. 2881-2902. MR2176963 (2006g:35144)

[16] D. Henry, Geometric Theory of Semilinear Parabolic Equations, Lecture Notes in Mathematics, 840 (1990), Springer. MR610244 (83j:35084)

[17] A.N. Kolmogorov, I.G. PetrovskiI, N.S. Piskunov, A study of the equation of diffusion with increase in the quantity of matter, and its application to a biological problem, Bjul. Moskowskogo Gos. Univ. 17 (1937), pp. 1-26.

[18] J.-F. Mallordy, J.-M. Roquejoffre, A parabolic equation of the KPP type in higher dimensions, SIAM J. Math. Anal. 26 (1995), pp. 1-20. MR1311879 (96a:35090)

[19] P. Martinez, J.-M. Roquejoffre, Rate of attraction of super-critical waves in a FisherKPP type equation with shear flow, preprint.

[20] P. Martinez, J.-M. Roquejoffre, Convergence to critical waves in KPP equations, in preparation.

[21] G. NADin, Traveling fronts in space-time periodic media, J. Math. Pures Appl. 92 (2009), pp. 232-262. MR 2555178 (2010i:35193)

[22] J.R. NorRIS, Long-time behaviour of heat flow: global estimates and exact asymptotics, Arch. Rational Mech. Anal., 140 (1997), pp. 161-195. MR1482931 (98k:35085)

[23] J. Ortega, E. Zuazua, Large time behavior in $R N$ for linear parabolic equations with periodic coefficients, Asymptotic Analysis 22 (2000), pp. 51-85. MR1739518 (2001b:35136)

[24] P. PolaČIK, E. YANAGIDA, On bounded and unbounded global solutions of a supercritical semilinear heat equation, Math. Ann. 327 (2003), pp. 745-771. MR.2023315 (2005b:35133) 
[25] J.-M. RoqUEJOFFre Eventual monotonicity and convergence to travelling waves for semilinear parabolic equations in cylinders, Ann. Inst. Henri Poincaré, Analyse non linéaire, 14 (1997), pp. 499-552. MR1464532 (98h:35107)

[26] J.-M. Roquejoffre, V. Roussier-Michon, Nontrivial large-time behaviour in bistable reaction-diffusion equations, Ann. Mat. Pura Appl. 188 (2009), pp. 207-233. MR 2491801 (2010k:35251)

[27] L. Rossi, Generalized principal eigenvalue in unbounded domains and applications to nonlinear elliptic and parabolic problems, Ph.D. Thesis, December, 2006.

[28] D. H. Sattinger, On the stability of waves of nonlinear parabolic systems, Advances in Math., 22 (1976), pp. 312-355. MR0435602 (55:8561)

[29] H.B. StewART, Generation of analytic semigroups by strongly elliptic operators under general boundary conditions, Trans. Amer. Math. Soc., 259 (1980), pp. 299-310. MR561838 (82h:35048)

[30] K. UCHIYAMA, The behavior of solutions of some nonlinear diffusion equations for large time, J. Math. Kyoto Univ. 18 (1978), pp. 453-508. MR.509494 (80g:35016)

[31] J.-L. Vézquez, E. Zuazua, Complexity of large time behaviour of evolution equations with bounded data, Chinese Annals of Mathematics, 23, ser. B (2002), 2, pp. 293-310. Special issue in honor of J.L. Lions. MR.1924144 (2003f:35175)

[32] E. YANAGIDA, Irregular behavior for solutions of Fisher's equation, Journal of Dynamics and Differential Equations, 19 (2007), pp. 895-914. MR2357530 (2009d:35168)

Institut de Mathématiques (UMR CNRS 5219), Université Paul Sabatier, 31062 Toulouse Cedex 4, France

Institut de Mathématiques (UMR CNRS 5219), Université Paul Sabatier, 31062 Toulouse Cedex 4, France

Institut de Mathématiques (UMR CNRS 5219), Université Paul Sabatier, 31062 Toulouse Cedex 4, France 